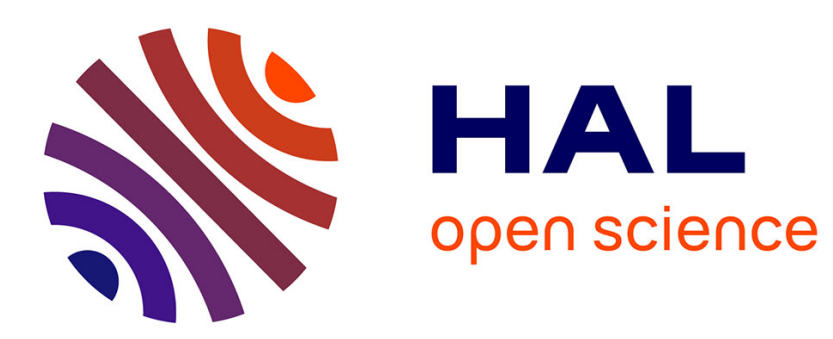

\title{
Modelling COVID-19 contagion: risk assessment and targeted mitigation policies
}

Rama Cont, Artur Kotlicki, Renyuan Xu

\section{To cite this version:}

Rama Cont, Artur Kotlicki, Renyuan Xu. Modelling COVID-19 contagion: risk assessment and targeted mitigation policies. 2020. hal-02923033v3

\section{HAL Id: hal-02923033 \\ https://hal.science/hal-02923033v3}

Preprint submitted on 9 Dec 2020

HAL is a multi-disciplinary open access archive for the deposit and dissemination of scientific research documents, whether they are published or not. The documents may come from teaching and research institutions in France or abroad, or from public or private research centers.
L'archive ouverte pluridisciplinaire HAL, est destinée au dépôt et à la diffusion de documents scientifiques de niveau recherche, publiés ou non, émanant des établissements d'enseignement et de recherche français ou étrangers, des laboratoires publics ou privés. 


\title{
Modelling COVID-19 Contagion: Risk Assessment and Targeted Mitigation Policies
}

\author{
Rama CONT
}

\author{
Artur KOTLICKI
}

Original version: August 2020.

Revised version: November 2020

\author{
Renyuan XU
}

\begin{abstract}
We use a spatial epidemic model with demographic and geographic heterogeneity to study the regional dynamics of COVID-19 across 133 regions in England.

Our model emphasises the role of variability of regional outcomes and heterogeneity across age groups and geographic locations, and provides a framework for assessing the impact of policies targeted towards sub-populations or regions. We define a concept of efficiency for comparative analysis of epidemic control policies and show targeted mitigation policies based on local monitoring to be more efficient than country-level or non-targeted measures. In particular, our results emphasise the importance of shielding vulnerable sub-populations and show that targeted policies based on local monitoring can considerably lower fatality forecasts and, in many cases, prevent the emergence of second waves which may occur under centralised policies.
\end{abstract}

Keywords: COVID-19; SARS-n-COV; metapopulation epidemic models; network model; compartmental models; SEIAR model; nowcasting. 


\section{Contents}

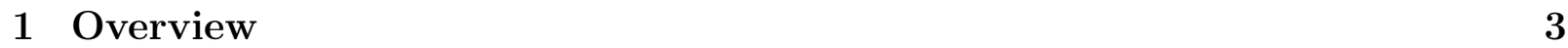

1.1 Methodology . . . . . . . . . . . . . . . . . . . 4

1.2 Summary of findings . . . . . . . . . . . . . . . . . . . . . . . 5

1.3 Outline . . . . . . . . . . . . . . . . . . . . . . . . . 6

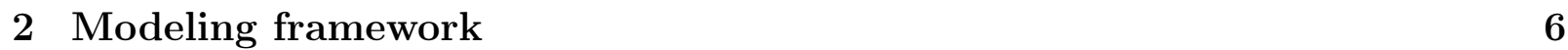

2.1 State variables . . . . . . . . . . . . . . . . . . . . . . . . . . . . 6

2.2 A metapopulation SEIAR model . . . . . . . . . . . . . . . . . . . . . 7

2.3 Stochastic dynamics . . . . . . . . . . . . . . . . . . . . . 9 9

2.4 Policies for epidemic control . . . . . . . . . . . . . . . . . . . . . . . . . . 9

3 Data sources and parameter estimation 11

3.1 Data sources. . . . . . . . . . . . . . . . . . . 11

3.2 Modeling of inter-regional mobility . . . . . . . . . . . . . . . . . . . . . . 12

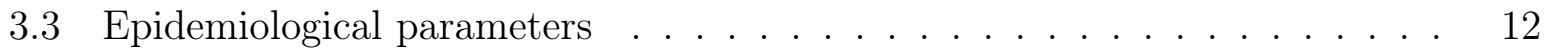

3.4 Estimation of the infection rate . . . . . . . . . . . . . . . . . . . . 15

3.5 Inter-regional mobility and social contact during confinement . . . . . . . . 16

3.6 Goodness-of-fit . . . . . . . . . . . . . . . . . . 17

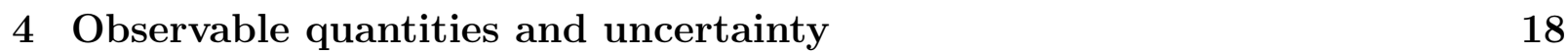

4.1 Observable quantities . . . . . . . . . . . . . . . . . . . . . . . . . . . 19

4.2 Implications of partial observability . . . . . . . . . . . . . . . . . . . . 20

5 Comparative analysis of epidemic control policies $\quad 22$

5.1 Confinement followed by social distancing . . . . . . . . . . . . . . . . . 22

5.2 Targeted policies . . . . . . . . . . . . . . . . . . . 26

5.2 .1 Pubs and schools . . . . . . . . . . . . . . . . 27

5.2 .2 Shielding of senior citizens . . . . . . . . . . . . . . . . . . 28

$6 \quad$ Adaptive mitigation policies $\quad 30$

6.1 Country-wide restrictions . . . . . . . . . . . . . . . . . . . . . . 30

6.2 Decentralised policies . . . . . . . . . . . . . . . . . . . . . . . . . . . . . . . . . . . . 34

6.3 Adaptive versus pre-planned policies . . . . . . . . . . . . . . . . . 39

\begin{tabular}{|l|l}
\hline A Demographic regions & 47 \\
\hline
\end{tabular}

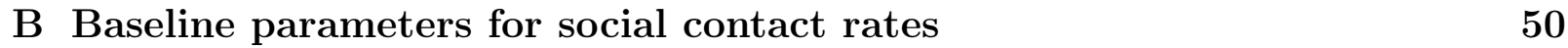




\section{Overview}

The novel coronavirus pandemic of 2019-2020 has led to disruption on a global scale, leading to more than 1.4 million deaths worldwide at the time of writing, and prompted the implementation of government policies involving a variety of 'non-pharmaceutical interventions' 22] including school closures, workplace restrictions, restrictions on social gatherings, social distancing and, in some cases, general lockdowns for extended periods. This has led to a range of different public health policies across the world, and the efficiency of specific policy choices has been subject to much debate.

While the nature of these restrictions has been justified by the severe threat to public health posed by the virus, their design and implementation necessarily involves a trade-off, often implicit in the decision-making process, between health outcomes and the socioeconomic impact of such social restrictions.

An important feature of the COVID-19 pandemic has been the heterogeneity of epidemic dynamics and the resulting mortality across different regions, age classes and population categories. The importance of these heterogeneities suggests that homogeneous models - often invoked in discussions on reproduction number and herd immunity - may provide misleading insights, and points to the need for more granular modeling to take into account geographic, demographic and social factors which may influence epidemic dynamics.

We propose a flexible modelling framework which can serve as a decision aid to policy makers and public health experts by quantifying this trade-off between health outcomes and social cost. Using a structured population model for epidemic dynamics which accounts for geographic and demographic heterogeneity, we formulate this trade-off as a control problem for a partially observed distributed system and provide a quantitative framework for comparative analysis of various mitigation policies. We illustrate the usefulness of the framework by applying it to the study of COVID-19 dynamics across regions in England and showing how it may be used to reconstruct the latent progression of the epidemic and perform a comparative analysis of various mitigation policies through scenario projections.

Several recent studies have used homogeneous compartmental models [3, 36, 17, 29, 44, 51, 48, 52] or age-stratified versions of such models [1, 14, 16, 34, 46, 55] to analyse the dynamics and impact of the COVID-19 epidemic in various countries. Our framework, while compatible with such homogeneous models at aggregate level, accounts for demographic and spatial heterogeneity in a more detailed manner, leading to regional outcomes which may substantially deviate from homogeneous models Similar, though somewhat less detailed, heterogeneous models have been recently used to study COVID-19 outbreaks by Danon et al. [15] for the UK, Birge et al. 9] for New York City and Roques et al. [50] for France.

We first present below an overview of the main features of our approach and the key findings, before going into more detail on the methodology and results. 


\section{$1.1 \quad$ Methodology}

We formulate a stochastic compartmental (SEIAR) epidemic model with spatial and demographic heterogeneity (age stratification) for modeling the dynamics of the COVID-19 epidemic and apply this model to the study of COVID-19 dynamics across regions in England.

The model takes into account:

- epidemiological features estimated by previous studies on COVID-19;

- the lack of direct observability of the total number of infectious cases and the presence of a non-negligible fraction of asymptomatic cases;

- the demographic structure of UK regions (age distribution, density);

- social contact rates across age groups derived from survey data;

- data on inter-regional mobility; and

- the presence of other random factors, not determined by the above.

We first demonstrate that this model is capable of accurately reproducing the early regional dynamics of the disease, both pre-lockdown and a month into lockdown, using a detailed calibration procedure that accounts for demographic heterogeneity across regions, low testing rates, and existence of asymptomatic carriers. The calibration reveals interesting regional patterns in social contact rates before and during lockdown.

Underlying any public health policy is a trade-off between a health outcome - which may relate to mortality or hospitalisations - and the socio-economic impact of measures taken to mitigate the magnitude of the impact on public health. We present an explicit formulation of this trade-off and use it to perform a comparative analysis of various 'social distancing' policies, based on two criteria:

- the benefit, in terms of reduction in projected mortality; and

- the cost, in terms of restrictions on social contacts.

The goal of our analysis is to make explicit the policy outcomes for decision-makers, without resorting to (questionable) concepts such as the 'economic value of human life' used in some actuarial and economic models [1, 44, 52].

In our comparative analysis, we consider a broad range of policies and pay particular attention to population-wide versus targeted mitigation policies, feedback control based on the number of observed cases and the benefits of broader testing. We introduce a concept of efficient policy, and show how this concept allows to identify decision parameters which lead to the most efficient outcomes for each type of mitigation policy. The granular nature of our model, together with validation based on epidemiological data, provide a more detailed picture of the relative merits of various public health policies. 


\subsection{Summary of findings}

Our first set of results concerns the reconstruction of the progression of the pandemic in England, in particular its latent spread through asymptomatic carriers.

- Using a baseline epidemic model consistent with epidemiological data and observations on fatalities and cases reported in England up to June 2020, we estimate more than 17.8 million persons in England (31.7\% of the population) to have been exposed to COVID-19 by August 1, 2020. These estimates are much higher than numbers discussed in media reports, based on the number of reported cases.

- Based on a comparison of fatality counts and reported cases, we infer that less than 5\% of cases in England had been detected prior to June 2020. This low detection probability implies in particular that the number of reported cases may severely underestimate the latent progression of the epidemic.

- We observe significant differences in epidemic dynamics across regions in England, with higher fatality and contagion levels in northern regions compared to southern regions, both before and during the lockdown period, pointing to the importance of demographic and geographic heterogeneity for modeling the impact of COVID-19.

After calibrating the model to replicate the regional progression of COVID-19 in England for the period March 1 to May 31, 2020, we use it for scenario projections under various mitigation policies. Comparative analysis of mitigation policies reveals that measures targeting sub-populations - such as regions with outbreaks - are more efficient than population-wide measures in terms of the tradeoff between health outcomes and social cost. More specifically:

- Shielding of elderly populations is by far the most effective single measure for reducing the number of fatalities 1

- By contrast, school closures and workplace restrictions are seen to be less effective than social distancing measures outside of school and work environments.

- Adaptive policies ('feedback control') which trigger measures when the number of daily observed cases exceed a threshold, are shown to be more effective than preplanned policies, leading to a substantial improvement in health outcomes. As such policies are based on monitoring of new cases, broader testing significantly improves their outcome.

- A decentralised policy which triggers regional confinement measures based on regional daily reported cases is found to be more efficient than centralised policies based on national indicators, resulting on average in an overall reduction of 20,000 in fatalities and, in many cases, significant damping of a 'second wave'.

\footnotetext{
${ }^{1}$ We do recognise that the implementation of such shielding measures may be extremely challenging in practice.
} 
- Comparative analysis of policies (Table 10) shows a wide range of health outcomes. The most effective policy in terms of reducing fatalities involves triggering of regional confinement measures decentralised based on monitoring of new cases, coupled with shielding of elderly populations.

The present work should be seen as an illustration of what may be done using our methodology, rather than an exhaustive analysis of different policy options and scenarios. We have made available an online implementation of the model, which may be used to explore other scenarios and policies than those presented below:

$$
\text { http://covid19.kotlicki.pl }
$$

\subsection{Outline}

The modeling framework is described in Section 2, Data sources and parameter estimations are detailed in Section 3. Section 4 highlights the implications of partial observability of state variables and the associated model uncertainty.

The outcomes of various epidemic control policies are then discussed in Sections 5 and 6 . Pre-planned policies are discussed in Sections 5.1 and 5.2 , while Section 6 discusses adaptive ('feedback') control policies, in which measures are triggered when the daily number of new reported cases exceeds a threshold, and concludes with a comparative analysis of health outcomes and social cost of various types of mitigation policies.

\section{Modeling framework}

To take into account the role of geographic and demographic heterogeneity, we use a stochastic compartmental (SEIAR) model with age stratification, mobility across sites, social contact across age stratification, and the impact of asymptomatic infected individuals. For general concepts on deterministic and stochastic compartmental models we refer to Anderson and May [5], Brauer and Castillo-Chavez [10], Britton et al. [11], Lloyd and Jansen [35].

\section{$2.1 \quad$ State variables}

We consider a regional meta-population model with $K$ regions labeled $r=1, \ldots, K$. Each region $r$ has a population $N(r)$ which is further subdivided into $M$ age classes labeled $a \in\{1,2,3,4, \ldots, M\}$. We denote $N(r, a)$ the population in region $r$ in age category $a$, with $\sum_{a=1}^{M} N(r, a)=N(r)$.

Individuals in each region and age group are categorized into six compartments:

- Susceptible $(S)$ individuals who have not yet been exposed to the virus;

- Exposed $(E)$ individuals who have contracted the virus but are not yet infectious. Exposed individuals may then become infectious after a certain incubation period;

- Infectious $(I)$ individuals who manifest symptoms; 
- Asymptomatic $(A)$ infectious individuals;

- Recovered $(R)$ individuals. In line with current experimental and clinical observations on COVID-19, we shall assume that individuals who have recovered have temporary immunity, at least for the horizon of the scenarios considered, and cannot be reinfected [7]; and

- Deceased $(D)$ individuals.

The progression of the disease in the population is monitored by keeping track of the respective number

$$
S_{t}(r, a), \quad E_{t}(r, a), \quad I_{t}(r, a), \quad A_{t}(r, a), \quad R_{t}(r, a), \quad D_{t}(r, a)
$$

of individuals in each compartment. As the model focuses on the dynamics of the epidemic over a short period (1000 days), we neglect demographic changes over this period and assume that the population size $N(r, a)$ in each location and age group is approximately constant, that is

$$
S_{t}(r, a)+E_{t}(r, a)+I_{t}(r, a)+A_{t}(r, a)+R_{t}(r, a)+D_{t}(r, a)=N(r, a)
$$

is constant.

\subsection{A metapopulation SEIAR model}

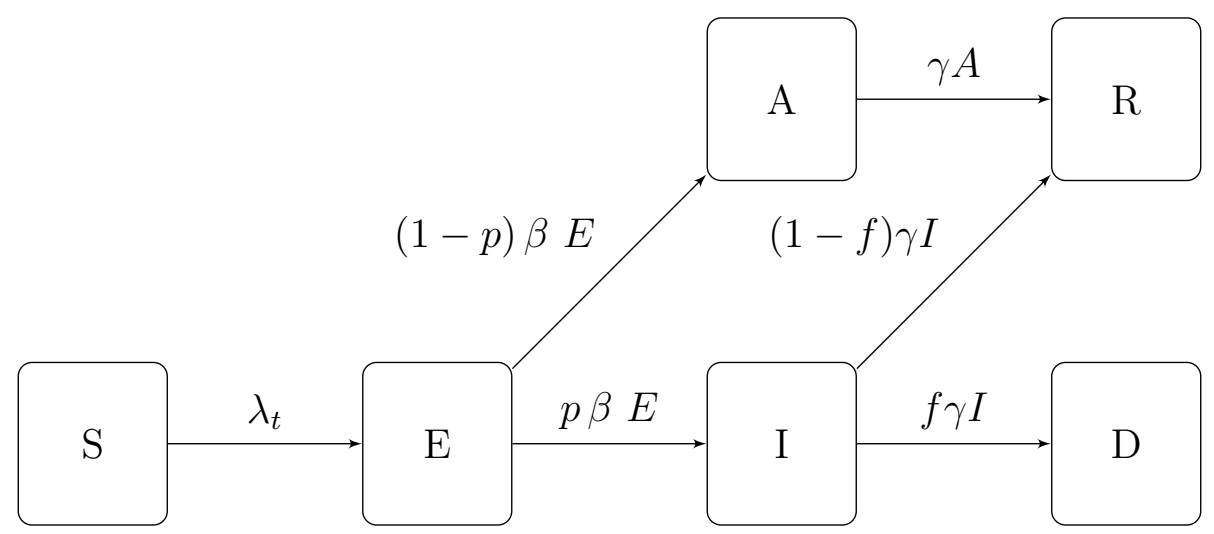

Figure 1: Epidemic dynamics. 
When each subpopulation $(r, a)$ is large and homogeneous, the dynamics of state variables may be described through the following system of equations, represented in Figure 1:

$$
\left\{\begin{array}{l}
\dot{S}_{t}(r, a)=-\lambda_{t}(r, a) S_{t}(r, a) \\
\dot{E}_{t}(r, a)=\lambda_{t}(r, a) S_{t}(r, a)-\beta E_{t}(r, a) \\
\dot{I}_{t}(r, a)=p_{a} \beta E_{t}(r, a)-\gamma I_{t}(r, a) \\
\dot{A}_{t}(r, a)=\left(1-p_{a}\right) \beta E_{t}(r, a)-\gamma A_{t}(r, a), \\
\dot{D}_{t}(r, a)=\gamma f_{a} I_{t}(r, a) \\
\dot{R}_{t}(r, a)=\gamma\left(1-f_{a}\right) I_{t}(r, a)+\gamma A_{t}(r, a) \\
N(r, a)=S_{t}(r, a)+A_{t}(r, a)+E_{t}(r, a)+I_{t}(r, a)+R_{t}(r, a)+D_{t}(r, a) .
\end{array}\right.
$$

where

- $0<\alpha<1$ is the infection rate per contact, that is the probability of infection conditional on contact;

- $\beta$ is the incubation rate, and $1 / \beta$ is the average incubation period;

- $\gamma$ is the rate at which infectious individuals recover;

- $0<p_{a}<1$ is the probability for an infected individual in age group $a$ to develop symptoms;

- $f_{a}$ is the infection fatality rate for age group $a$, representing the probability that an infected individual in age group $a$ dies from the disease; and

- The force of infection $\lambda_{t}(r, a)$, which measures the rate of exposure at location $r$ for age group $a$, is given by

$$
\begin{aligned}
\lambda_{t}(r, a)= & \alpha \sum_{a^{\prime} \notin \mathcal{W}} \sigma_{a, a^{\prime}}^{r}(t) \frac{\kappa I_{t}\left(r, a^{\prime}\right)+A_{t}\left(r, a^{\prime}\right)}{N\left(r, a^{\prime}\right)} \\
& +\alpha \sum_{a^{\prime} \in \mathcal{W}} \sigma_{a, a^{\prime}}^{r}(t) \sum_{r^{\prime}=1}^{K} M_{r, r^{\prime}}(t) \frac{\kappa I_{t}\left(r^{\prime}, a^{\prime}\right)+A_{t}\left(r^{\prime}, a^{\prime}\right)}{N\left(r^{\prime}, a^{\prime}\right)} .
\end{aligned}
$$

The force of infection in each subpopulation $(r, a)$ depends on the rate of contact with (infected) individuals in other subpopulations, which differentiates this model from a homogeneous model. These interactions occur through:

- Contacts across age groups in the same region: the term $\sigma_{a, a^{\prime}}^{r}(t)$ represents the average number of persons from age class $a^{\prime}$ encountered per day by a person from age class $a$ in region $r$ on a day $t$. For infectious individuals with symptoms, we assume a lower contact rate $\kappa \sigma<\sigma$ due to (partial) self-isolation. This leads to the first term in $(2.2)$.

- Inter-regional mobility: the second term in (2.2) corresponds to contacts between individuals in region $r$ and age class $a$ and those in the working population (age classes $\left.a^{\prime} \in \mathcal{W}\right)$ commuting from other regions $r^{\prime} \neq r . M_{r, r^{\prime}}(t)$ represents the proportion of individuals from region $r^{\prime}$ among the population of adults at a location $r$ at date $t$. 


\subsection{Stochastic dynamics}

The deterministic dynamics (2.1) ignores the variability of outcomes [25] due to random factors not taken into account in the model. To account for this variability of outcomes we model the variables $(S(t), E(t), I(t), A(t))$ as a continuous-time Markov point process [4, 11] defined through its transition rates conditional on the history $\mathcal{H}_{t}$ up to date $t$ :

$$
\left\{\begin{array}{l}
\mathbb{P}\left(\Delta S_{t}(r, a)=-1 \mid \mathcal{H}_{t}\right)=-\lambda_{t}(r, a) S_{t}(r, a) \Delta t+o(\Delta t) \\
\mathbb{P}\left(\Delta E_{t}(r, a)=1 \mid \mathcal{H}_{t}\right)=\lambda_{t}(r, a) S_{t}(r, a) \Delta t+o(\Delta t) \\
\mathbb{P}\left(\Delta E_{t}(r, a)=-1 \mid \mathcal{H}_{t}\right)=\beta E_{t}(r, a) \Delta t+o(\Delta t) \\
\mathbb{P}\left(\Delta I_{t}(r, a)=1 \mid \mathcal{H}_{t}\right)=p_{a} \beta E_{t}(r, a) \Delta t+o(\Delta t) \\
\mathbb{P}\left(\Delta I_{t}(r, a)=-1 \mid \mathcal{H}_{t}\right)=\gamma I_{t}(r, a) \Delta t+o(\Delta t) \\
\mathbb{P}\left(\Delta A_{t}(r, a)=+1 \mid \mathcal{H}_{t}\right)=\left(1-p_{a}\right) \beta E_{t}(r, a) \Delta t+o(\Delta t) \\
\mathbb{P}\left(\Delta A_{t}(r, a)=-1 \mid \mathcal{H}_{t}\right)=\gamma A_{t}(r, a) \Delta t+o(\Delta t) \\
\mathbb{P}\left(\Delta D_{t}(r, a)=1 \mid \mathcal{H}_{t}\right)=f_{a} \gamma I_{t}(r, a) \Delta t+o(\Delta t)
\end{array}\right.
$$

The stochastic dynamics (2.3) are consistent with the deterministic dynamics of (2.1) for large populations, in the sense that the population fractions represented by each compartment converge to those represented by the solution of (2.1) as $\min _{r} N(r)$ increases. However, even when the overall population is large, the stochastic dynamics (2.3) can substantially deviate from the deterministic model (2.1), especially in small subpopulations and in the early phases of the epidemic when the number of infected individuals in each region may be small, leading to random flare-ups and breakouts not present in the deterministic model. In the sequel we use the stochastic model 2.3 for the dynamics of the state variables.

\subsection{Policies for epidemic control}

Social distancing policies (and lockdowns) affect epidemic dynamics by influencing (lowering) the social contact rates $\sigma_{i j}^{r}$ and the inter-regional mobility $M_{r, r^{\prime}}$. To discuss targeted policies which may influence differently social contact rates at different locations, we decompose the baseline social contact matrix $\sigma^{r}$ as

$$
\sigma^{r}(0)=\sigma^{r, H}+\sigma^{r, W}+\sigma^{r, S}+\sigma^{r, O},
$$

where the components correspond respectively to contacts at home $\left(\sigma^{r, H}\right)$, at work $\left(\sigma^{r, W}\right)$, school $\left(\sigma^{r, S}\right)$ and other locations $\left(\sigma^{r, O}\right)$. Social distancing policies are then parameterised in terms of their impact on various components of the social contact matrix:

$$
\sigma_{i j}^{r}(t)=u_{i j}^{r, H}(t) \sigma_{i j}^{r, H}+u_{i j}^{r, S}(t) \sigma_{i j}^{r, S}+u_{i j}^{r, W}(t) \sigma_{i j}^{r, W}+u_{i j}^{r, O}(t) \sigma_{i j}^{r, O} \leq \sigma_{i j}^{r}(0),
$$

where $0 \leq u_{i j}^{r, X}(t) \leq 1$ are modulating factors which measure the impact of the policy on social contacts between age groups $i$ and $j$ at a location $X$ in region $r$. In absence of social distancing or confinement measures, we have $u_{i j}^{r, X}(t)=1$; the value of $u_{i j}^{r, X}(t)$ reflects the 
fraction of social contacts when between age groups $i$ and $j$ at location $X$ in region $r$ when the policy is applied.

This parameterisation allows us to consider policies targeted towards sub-population or specific regions. For example, school closure in region $r$ during time period $\left[t_{1}, t_{2}\right]$ corresponds to setting $u_{i j}^{r, S}(t)=0$ for $t \in\left[t_{1}, t_{2}\right]$, while $0<u_{i j}^{r, S}<1$ corresponds to social distancing in schools, with lower values of $u_{i j}^{r, S}$ corresponding to stricter enforcement of measures.

In most cases $u_{i j}^{r, X}(t)$ does not explicitly depend on the age groups $i, j$ as it is infeasible to discriminate between age groups when implementing social distancing requirements. Dependence on age groups arises when certain types of contacts are primarily related to certain age groups:

- Shielding of elderly populations: such policies affect the contact rates between elderly populations and other age groups.

- Work restrictions, which affect contacts between age groups of the working population $($ denoted $\mathcal{W}): u_{i j}^{r, W}(t)=u^{r, W} \mathbb{1}_{i \in \mathcal{W}} \mathbb{1}_{j \in \mathcal{W}}$.

Regarding the inter-regional mobility matrix $M$, following the interpretation discussed in Section 3.2, we modulate its value according to the fraction $u^{r, W}$ of the population who continue to commute, that is

$$
M_{r, r^{\prime}}(t)=u^{r, W}(t) M_{r, r^{\prime}}+\left(1-u^{r, W}(t)\right) \mathbf{I} .
$$

Here $M_{r, r^{\prime}}$ is the fraction of population in region $r$ whose habitual residence is in region $r^{\prime}$.

The modulating factors $u_{i j}^{r, X}$ may be chosen in advance or expressed as a function of the state of the system. We distinguish:

- Pre-planned (also called 'open-loop') policies, in which target values of modulating factors $u_{i j}^{r, X}(t)$ are decided in advance; and

- Adaptive policies (also called 'closed loop' or feedback control), in which actions are decided and updated as a function of observed quantities such as number of daily reported cases or number of daily fatalities.

Comparative analysis of mitigation policies To perform comparative analysis across different policies, we need to evaluate policy outcomes across two dimensions: health outcome and socio-economic impact.

We quantify the health outcome of each policy by the total number of fatalities during a reference period, taken to be $t_{\max }=1000$ days after the reference date of March 1, 2020 . The length of this reference period is chosen such that it takes into account an eventual 'second wave' of fatalities. We denote this outcome by $D_{t_{\max }}(u)$, which represent the total fatalities at date $t_{\max }$ associated with policy $u$.

To quantify the socio-economic impact of a policy, we use as metric the reduction in social contact resulting from the policy over the horizon $\left[0, t_{\max }\right]$, that is

$$
J(u)=\sum_{t=1}^{t_{\max }} \sum_{r=1}^{K} \sum_{i, j=1}^{M}\left(\sigma_{i j}^{r}(0)-\sigma_{i j}^{r}(t)\right) N(r, i),
$$


defined in terms of person $\times$ day units.

The range of policies examined below lead to different outcomes in terms of fatalities $D_{t_{\max }}(u)$ and social cost $J(u)$. A policy $v$ dominates (or improves upon) a policy $u$ if it leads to a similar or better health outcome at an equal or lower cost:

$$
J(v) \leq J(u) \quad \text { and } \quad D_{t_{\max }}(v) \leq D_{t_{\max }}(u)
$$

with at least one inequality being strict. A policy $u$ is efficient among a class of policies $U$ if it cannot be improved upon by any policy in this class. Given a set of policies $U$, the subset of efficient policies forms the efficient frontier of $U$.

Some recent economic models [1, 44, 52] formulate the trade-off in different terms, by introducing a concept of monetary value of human life in order to build a (monetary) welfare function combining both terms. Aside from ethical issues linked to the very concept of monetisation of human life, there is no consensus on its actual value, which is a key determinant of the trade-off in this approach. Our approach avoids specifying such a value and aims at identifying the range of efficient policies, leaving the final choice of the trade-off to policymakers.

In what follows, the goal is to determine the set of efficient policies and describe the characteristics and outcomes of such policies. Pre-planned policies are discussed in Sections 5.1 and 5.2, while adaptive policies are discussed in Section 6 .

\section{Data sources and parameter estimation}

We now describes the model inputs as well as the methodology used in the parameter estimation. Table 4 contains a summary of model parameters.

\subsection{Data sources}

The basic inputs of the model are panel data on number of cases and fatalities reported at the level of Upper Tier Local Authorities (UTLA) level in England, provided by the Public Health England and NHSX [47]. This defines the geographic granularity of the model: we partition the population of England into 133 regions as defined by the Nomenclature of Territorial Units for Statistics at level 3 (NUTS-3) [20].

For the purpose of our study we distinguish $M=16$ age groups, as shown in Table 1 . which is the maximum granularity allowed by the available estimates of age-dependent social contact rates and fatality rates. The size $N(r, a)$ of age group $a$ in region $r$ is

retrieved using the population dataset provided by Eurostat [19]. Appendix A provides the list of UK regions used in this study and outlines the performed mapping procedure from UTLA to NUTS-3 regions to ensure consistency across data sources. 


\begin{tabular}{|l|l|l|l|l|l|l|l|l|}
\hline Age group & {$[0,5)$} & {$[5,10)$} & {$[10,15)$} & {$[15,20)$} & {$[20,25)$} & {$[25,30)$} & {$[30,35)$} & {$[35,40)$} \\
\hline Size (millions) & 3.3 & 3.5 & 3.3 & 3.1 & 3.5 & 3.8 & 3.8 & 3.7 \\
Fraction & $5.9 \%$ & $6.3 \%$ & $5.9 \%$ & $5.5 \%$ & $6.2 \%$ & $6.8 \%$ & $6.8 \%$ & $6.6 \%$ \\
\hline \hline Age group & {$[40,45)$} & {$[45,50)$} & {$[50,55)$} & {$[55,60)$} & {$[60,65)$} & {$[65,70)$} & {$[70,75)$} & {$[75,100)$} \\
\hline Size (millions) & 3.4 & 3.8 & 3.9 & 3.6 & 3.1 & 2.8 & 2.8 & 4.7 \\
Fraction & $6.0 \%$ & $6.7 \%$ & $7.0 \%$ & $6.5 \%$ & $5.5 \%$ & $5.0 \%$ & $4.9 \%$ & $8.4 \%$ \\
\hline
\end{tabular}

Table 1: Age group distribution for England, 2019. Source: Eurostat [19].

\subsection{Modeling of inter-regional mobility}

For our baseline estimate of inter-regional mobility we use the 2011 Census data on location of usual residence and place of work in the United Kingdom, provided by the Office for National Statistics [42. The dataset classifies people aged 16 and over in employment during March 2011 and shows the movement between their area of residence and workplace, defined in Local Administrative Units at level 1 (LAU-1) terms. We then map this data onto NUTS-3 regions using the lookup table between LAU-1 and NUTS-3 areas provided by the Office for National Statistics [43].

The data is then represented in the model through the inter-regional mobility matrix $M$, whose elements $M_{r, j}$ represent the fraction of population in region $r$ whose habitual residence is in region $j$. Denote by $\Pi(r, j)$ the population with residence registered in region $j$ and workplace registered in region $r$ for $r \neq j$. In addition, we denote by $\Pi(r, r)=$ $\sum_{a \in \mathcal{W}} N(a, r)$, where $\mathcal{W}=\{5,6,7,8,9,10,11,12\}$, the total population at location $r$ in the age category $[20,60)$ years. Then, we estimate the coefficients of $M_{r, j}$ by

$$
\widehat{M}_{r, j}=\frac{\Pi(r, j)}{\sum_{i=1}^{K} \Pi(r, i)} .
$$

\subsection{Epidemiological parameters}

Epidemiological parameters were either estimated from publicly available sources [2, 39] or set to values consistent with recent clinical and epidemiological studies in COVID-19 [18, 27, 56].

Social contact rates Contact rates across age classes have been estimated in studies by Mossong et al. [38, 39] and Béraud et al. [8]. We use the estimates of social contact rates provided by Mossong et al. [38, for the 16 age groups defined in Table 1. Using the PyRoss methodology [2], we further decompose the contact matrix, as in (2.4), into four components representing contacts at home $\left(\sigma^{H}\right)$, work $\left(\sigma^{W}\right)$, school $\left(\sigma^{S}\right)$ and other locations $\left(\sigma^{O}\right)$. Estimation methods and parameter values for these matrices are discussed in Appendix B.

Contact rates may vary across different regions due to the heterogeneity in socioeconomic composition structure and specific regional characteristics, such as population density, level of urbanisation and the level of use of public transport. To account for 
this heterogeneity, we parameterise the (pre-lockdown) contact matrix in region $r$ as $\sigma^{r}(0)=d_{r} \sigma$ where the regional adjustment factors $\left\{d_{r}: r=1, \ldots, 133\right\}$ are estimated to reproduce the regional growth rate of reported cases before the lockdown period. The results are displayed in Figure 2, Table 2 provides a summary of selected characteristics of five regions with the highest values of the regional adjustment factors $d_{r}$.

\begin{tabular}{|l|l|l|l|l|l|}
\hline Region & $d_{r}$ & Density & Inward mobility & Outward mobility & Population \\
\hline \hline UKC12 & 1.80 & $925.9(\# 59)$ & $17.6 \%(\# 82)$ & $19.1 \%(\# 105)$ & $276988(\# 102)$ \\
\hline UKI62 & 1.68 & $4518.4(\# 67)$ & $16.6 \%(\# 87)$ & $43.2 \%(\# 12)$ & $389473(\# 59)$ \\
\hline UKG32 & 1.64 & $1205.5(\# 59)$ & $14.5 \%(\# 96)$ & $46.2 \%(\# 6)$ & $215055(\# 120)$ \\
\hline UKI53 & 1.62 & $6161.9(\# 11)$ & $23.4 \%(\# 50)$ & $41.6 \%(\# 16)$ & $587575(\# 25)$ \\
\hline UKC23 & 1.52 & $2026.9(\# 49)$ & $13.7 \%(\# 103)$ & $24.0 \%(\# 78)$ & $277733(\# 99)$ \\
\hline
\end{tabular}

Table 2: Summary of regions with the highest regional multiplier $d_{r}$ for social contact matrix. Number in brackets signify the respective rank of the measured quantity.

As seen in Figure 2, our findings imply heterogeneity of social contact rates across regions. As we will observe below, these differences have a considerable impact on regional epidemic dynamics.

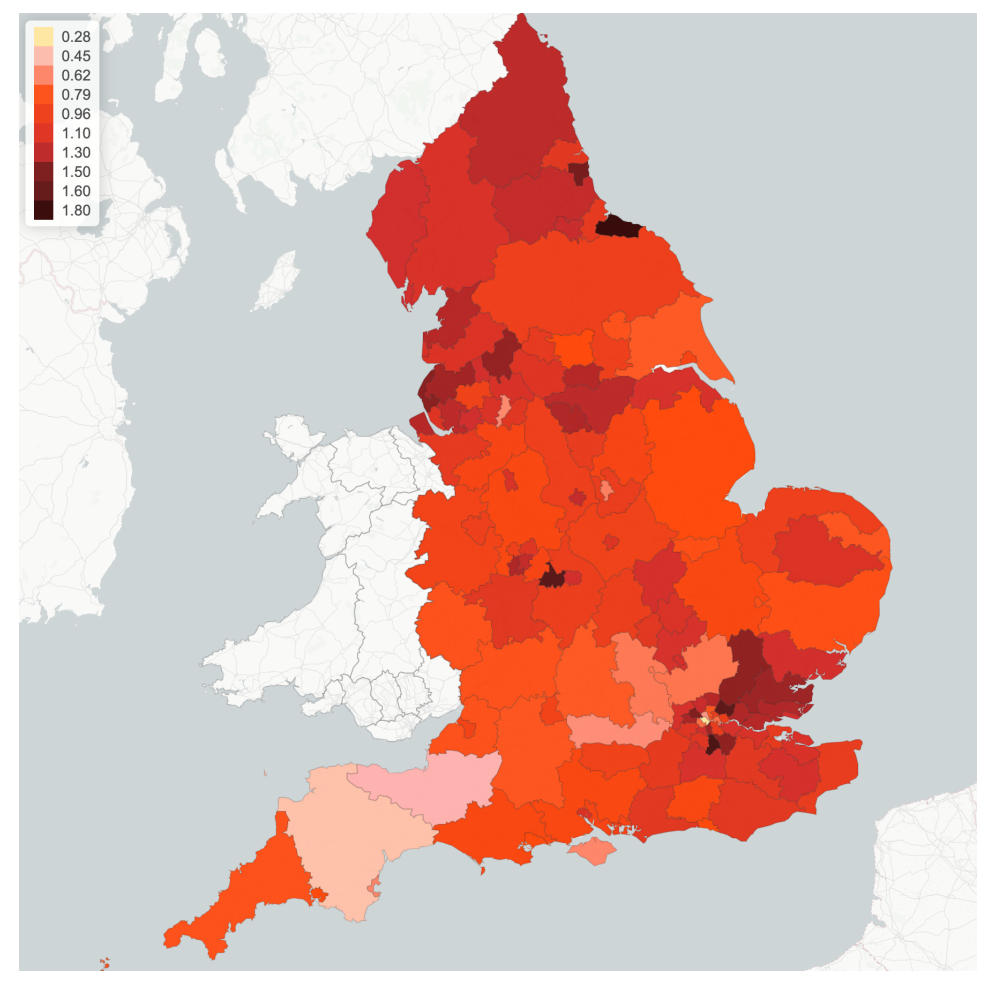

Figure 2: Regional multiplier $d_{r}$ for social contact matrix, implied by epidemic dynamics pre-lockdown (before March 23, 2020).

Incubation rate Following the study of Ferguson et al. [22], we use an incubation rate $\beta=0.2$, which corresponds to an incubation period of approximately 5 days. This is 
further supported by several empirical studies on diagnosed cases in China outside Hubei province. An early study of Backer et al. [6] based on 88 confirmed cases, which uses data on known travel to and from Wuhan to estimate the exposure interval, indicates a mean incubation period of 6.4 days with a $95 \%$ confidence interval (CI) of 5.6-7.7 days. Linton et al. [32, based on 158 confirmed cases, estimate a median incubation period of 5.0 days with $95 \%$ CI of 4.4-5.6 days and estimate the incubation period to have a mean of around 5 days with $95 \%$ CI of 4.2-6.0 days. Lauer et al. 30] estimates a median of incubation period to be 5.1 days with $95 \%$ CI of 4.5-5.8 days, based on 181 cases over the period of January 4 to February 24, 2020.

Proportion of symptomatic and asymptomatic infections The probability $p$ that an infected individual develops symptoms is an important parameter for epidemic dynamics, yet subject to a high degree of uncertainty: studies on various data sets [12, 16, 37, 23, 41] are based on small samples and yield a wide range of estimates. In particular, an early estimates from the Diamond Princess cruise ship [37] and Japanese evacuation flights from Wuhan yielded estimates as high as $p \simeq 0.7-0.8$ [40], while a July 2020 study by the Office for National Statistics [41], based on a much larger sample, showed that $p$ can be as low as 0.23 . However, clinical studies [16] indicate that this probability may strongly depend on the age group considered.

We use a range of values for the age-dependent probability $p_{a}$ whose upper bound is consistent with Davies et al. [16] and whose lower bound is consistent with the estimates provided by the Office for National Statistics [41]. These values are displayed in Table 3. Given the much larger sample size used in the study of Office for National Statistics [41], we use the corresponding estimates ('low values', denoted as $p_{\text {low }}$ in Table 3) as benchmark unless stated otherwise.

\begin{tabular}{|l|l|l|l|l|l|l|l|l|}
\hline Age group & {$[0,5)$} & {$[5,10)$} & {$[10,15)$} & {$[15,20)$} & {$[20,25)$} & {$[25,30)$} & {$[30,35)$} & {$[35,40)$} \\
\hline$p_{\text {low }}$ & 0.075 & 0.075 & $0 \cdot 05$ & $0 \cdot 05$ & 0.15 & 0.15 & 0.21 & 0.21 \\
$p_{\text {high }}$ & 0.15 & 0.15 & 0.1 & 0.1 & 0.3 & 0.3 & 0.42 & 0.42 \\
\hline \hline Age group & {$[40,45)$} & {$[45,50)$} & {$[50,55)$} & {$[55,60)$} & {$[60,65)$} & {$[65,70)$} & {$[70,75)$} & {$[75,100)$} \\
\hline$p_{\text {low }}$ & 0.23 & 0.23 & 0.28 & 0.28 & 0.41 & 0.41 & 0.375 & 0.375 \\
$p_{\text {high }}$ & 0.45 & 0.45 & 0.56 & 0.56 & 0.82 & 0.82 & 0.75 & 0.75 \\
\hline
\end{tabular}

Table 3: Age-dependent symptomatic ratios, $p$. Source: Office for National Statistics [41] and Davies et al. [16].

Recovery rate $\gamma$ In line with Cao et al. [13], Li et al. [31] and Rocklöv et al. [49, we use a recovery rate $\gamma=0.1$, which corresponds to an average infectious period of 10 days.

Infection fatality rates We denote by $f_{a}$ the (infection) fatality rate for age group $a$. In practice, these parameters are difficult to estimate during outbreaks and estimates may be subject to various biases [33]. Note that the infection fatality rate (IFR) is different from (and generally much smaller than) the case fatality rate. 
Fatality rates for COVID-19 have been observed to be highly variable across age groups [27, 53, 56]. Based on the infection fatality rates provided in Verity et al. [56] for different age groups and the UK population distribution, we derive the aggregated IFR for the respective 16 age groups of interest as summarised in Table 5. These estimates are consistent with data obtained from other countries; for example, see Salje et al. [53].

\begin{tabular}{|c|c|c|c|}
\hline Model parameter name & Symbol & Value & Source \\
\hline Infection rate & $\alpha$ & $\begin{array}{ll}0.055 \quad(0.051,0.062) \\
\end{array}$ & [17, 18] \\
\hline Incubation rate & $\beta$ & 0.2 & {$[22,30,32$} \\
\hline Recovery rate & $\gamma$ & 0.1 & [13, 31, 49] \\
\hline Infection fatality rate & $f$ & See Table 5 & 56 \\
\hline Symptomatic ratios (low estimate) & $p_{\text {low }}$ & Table 3 & 41 \\
\hline Symptomatic ratios (high estimate) & $p_{\text {high }}$ & Table 3 & 16 \\
\hline Social contact matrix & $\sigma$ & Appendix $B$ & 38 \\
\hline Symptomatic contact adjustment & $\kappa$ & 0.5 & \\
\hline $\begin{array}{l}\text { Regional adjustment for contact rates } \\
\text { (pre-lockdown) }\end{array}$ & $d_{r}$ & Figure 2 & \\
\hline $\begin{array}{l}\text { Regional adjustment for contact rates } \\
\text { (pre-lockdown) }\end{array}$ & $l_{r}$ & Table 6 & \\
\hline Inter-regional mobility matrix & $M$ & & 42 \\
\hline
\end{tabular}

Table 4: Summary of parameters for the COVID-19 model.

\begin{tabular}{|l|l|l|l|l|l|l|l|l|}
\hline Age group & {$[0,5)$} & {$[5,10)$} & {$[10,15)$} & {$[15,20)$} & {$[20,25)$} & {$[25,30)$} & {$[30,35)$} & {$[35,40)$} \\
\hline$f(\%)$ & 0.002 & 0.002 & 0.01 & 0.01 & 0.05 & 0.05 & 0.1 & 0.1 \\
\hline \hline Age group & {$[40,45)$} & {$[45,50)$} & {$[50,55)$} & {$[55,60)$} & {$[60,65)$} & {$[65,70)$} & {$[70,75)$} & {$[75,100)$} \\
\hline$f(\%)$ & 0.2 & 0.2 & 0.6 & 0.6 & 2.00 & 2.00 & 4.0 & 7.5 \\
\hline
\end{tabular}

Table 5: Age-dependent infection fatality rates. Source: Verity et al. [56].

\subsection{Estimation of the infection rate}

We use a simulation-based indirect inference method [24] for estimating the infection rate $\alpha$. We simulate the stochastic model $(2.3)$ for a range of values $0.03 \leq \alpha \leq 0.15$. The value of $\alpha$ is estimated by matching the logarithmic growth rates of the simulated reported cases with that of reported cases $C_{t}$ in England.

For the simulation, we use parameters specified in Table 4 and the following initial conditions for $t_{0}=$ March 10, 2020:

$$
E_{t_{0}}(r, a)=\frac{N(r, a)}{\sum_{a^{\prime} \in \mathcal{W}} N\left(r, a^{\prime}\right)} \frac{C_{t_{0}+5}(r)}{p_{2} \pi} 1_{a \in \mathcal{W}}
$$

where $\mathcal{W}=\{5,6,7,8,9,10,11,12\}$ corresponds to age groups in the working population and $A_{t_{0}}(r, a)=0, D_{t_{0}}(r, a)=0, I_{t_{0}}(r, a)=0$ for all $a$. These initial conditions ensure that 
the simulations agree on average with regional case numbers on March 15, 2020, for all values of $\alpha$.

This procedure yields an estimated value of $\widehat{\alpha}=0.055$ and a confidence interval $[0.051,0.062]$. This value of $\widehat{\alpha}$, together with the model parameters in Table 4, yields a good fit of the pre-lockdown evolution of case numbers.

These results are consistent with estimates obtained in Donnat and Holmes [17] and Dorigatti et al. [18] using data from other countries.

\subsection{Inter-regional mobility and social contact during confinement}

Confinement measures were implemented across the United Kingdom starting March 23, 2020 via the Coronavirus Act ${ }^{2}$. During this 'lockdown' period schools and workplaces were closed and social contact was reduced, as evidenced by mobility data..$^{3}$ However mobility data also reveal regional differences in the impact of the lockdown.

We model the reduction in inter-regional mobility through an adjusted mobility matrix

$$
\widehat{M}_{r, r^{\prime}}(t)=q \widehat{M}_{r, r^{\prime}}+(1-q) I, \quad \text { where } \quad 0<q<1
$$

and $\widehat{M}_{r, r^{\prime}}$ is the inter-regional mobility matrix defined in (3.1). According to the Labor Force Survey data from 2018/19 [21], 7.1 million adults across the UK are considered as 'key workers'. We set $q=20 \%$ to take into account the fact that these key workers continued to access their workplace during the lockdown period. This is also consistent with the methodology in Rawson et al. [48] and empirical studies of Santana et al. [54] on mobility changes before and after lockdown in the UK. Figure 3 shows the submatrix corresponding to daily mobility across London boroughs, and illustrates the observed dramatic drop in commute patterns.

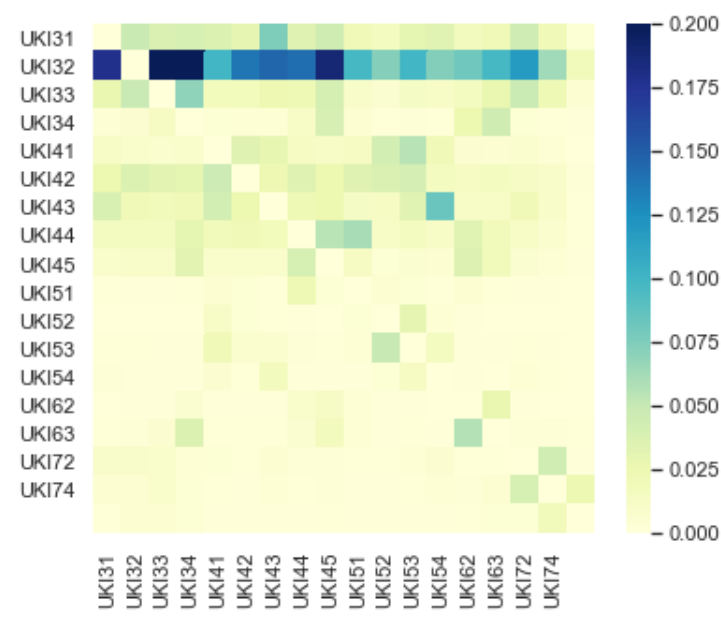

(a) Pre-lockdown: before March 23, 2020.

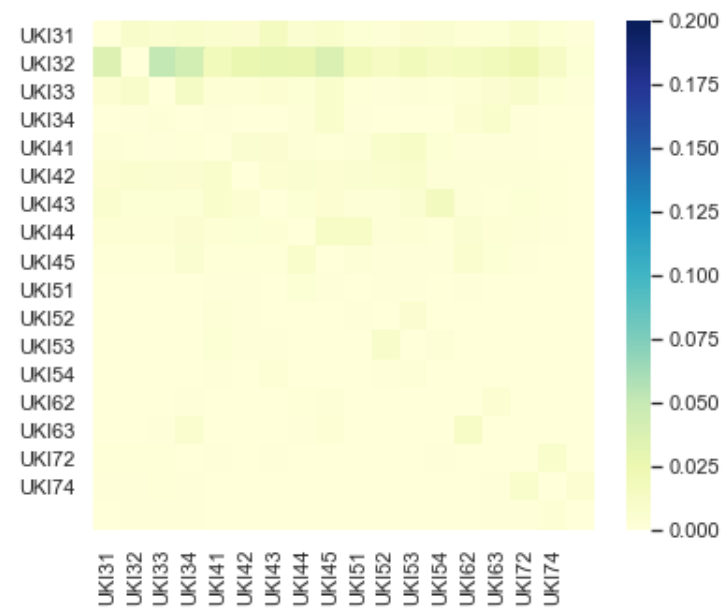

(b) During lockdown: 23/03-10/06/2020.

Figure 3: Inter-regional mobility across London boroughs.

\footnotetext{
${ }^{2}$ See https://www.legislation.gov.uk/ukpga/2020/7/contents/enacted.

${ }^{3}$ See https://www.oxford-covid-19.com/
} 
We model the impact of confinement on the social contact matrix through a regional multiplier $l_{r}$,

$$
\sigma^{r}(t)=l_{r} \times \sigma(0)
$$

where $l_{r} \leq d_{r}$ represents the reduction in social contacts during the lockdown period; $l_{r}=d_{r}$ corresponds to the pre-lockdown level of social contact. The value of $l_{r}$ is estimated from panel data on regional epidemic dynamics during the period from March 23 to June 1, 2020, using a least-squares logarithmic regression on the number of observed regional cases.

The average value of this reduction factor is found to be

$$
\frac{\sum_{r=1}^{133} N(r) l_{r}}{\sum_{r=1}^{133} N(r)}=0.12
$$

which is an average reduction of $88 \%$ in social contacts, an order of magnitude corroborated by mobility data [54], showing that the lockdown was very effective in reducing social contacts.

\begin{tabular}{|l|c|c|}
\hline NUTS-1 Region & Pre-lockdown $\left(d_{r}\right)$ & Lockdown $\left(l_{r}\right)$ \\
\hline \hline South West (UKK) & 0.729 & 0.099 \\
East Midlands (UKF) & 0.952 & 0.134 \\
London (UKI) & 1.143 & 0.100 \\
West Midlands (UKG) & 1.020 & 0.126 \\
Yorkshire and Humber (UKE) & 1.069 & 0.137 \\
South East (UKJ) & 0.920 & 0.116 \\
North East (UKC) & 1.260 & 0.131 \\
North West (UKD) & 1.122 & 0.137 \\
East of England (UKH) & 0.994 & 0.129 \\
\hline
\end{tabular}

Table 6: Estimated values for regional adjustments $d_{r}$ and $l_{r}$ in NUTS-1 regions.

\subsection{Goodness-of-fit}

Having estimated the model parameters using data on reported cases between March 10 and May 20, 2020 we assess the goodness-of-fit and out-of-sample performance using reported cases and fatalities between May 21 and June 22, 2020. Figures 4 and 5 show that the model is able to reproduce the in-sample and out-of-sample evolution of case numbers and fatalities, at national level as well as regional level. 


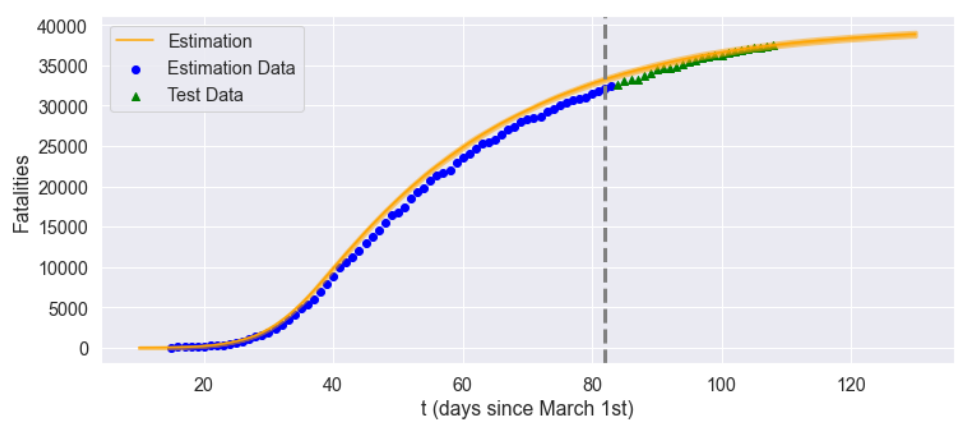

Figure 4: Fatalities in England: comparison of model with data. Grey dashed line: separation between estimation sample and test data; orange line: model simulation; blue dot: in-sample data; green triangle: out-of-sample data.

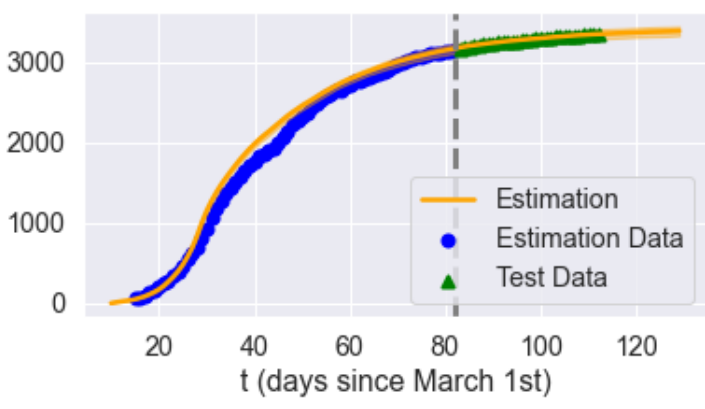

(a) Birmingham.

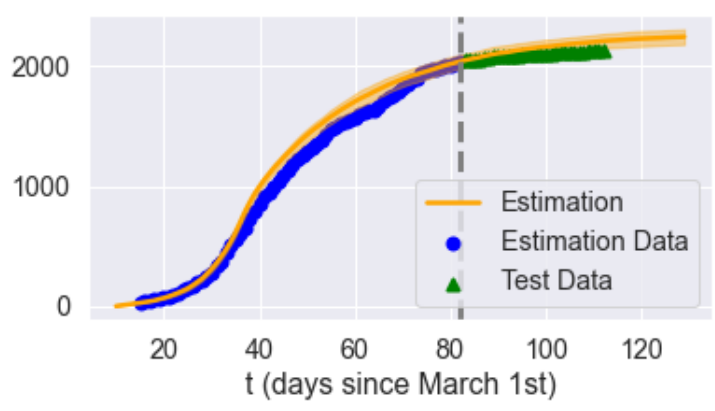

(c) Oxfordshire.

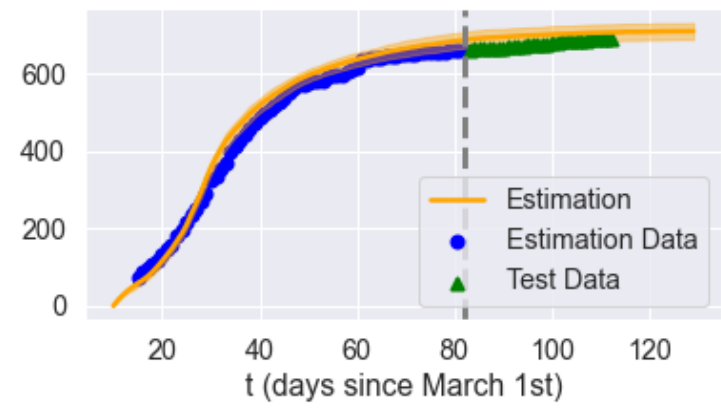

(b) Westminster.

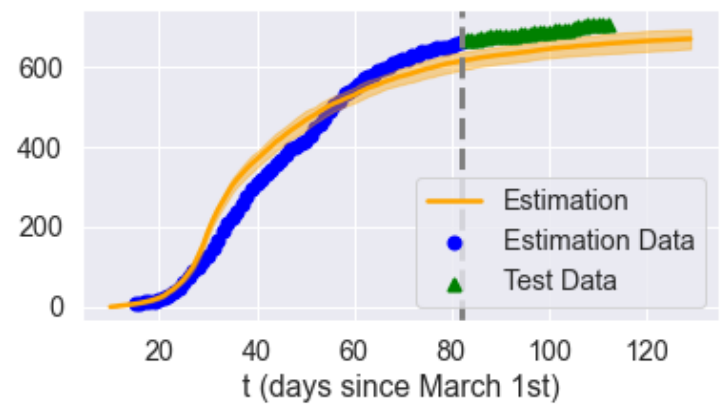

(d) West Essex.

Figure 5: Cumulative reported cases in selected regions. Grey dashed line: separation between estimation sample and test data; orange line: average of 50 simulated scenarios; blue dot: in-sample data; green triangle: out-of-sample data.

\section{Observable quantities and uncertainty}

When applying such models to epidemic data, a key point is to realize that the state variables $S, E, I, A, R$ are not directly observed (and certainly not in real time) but need to be inferred from other observable quantities. 
In absence of widespread testing, public health authorities are faced with the problem of controlling a system under partial observation. This lack of direct observability has some implications for the estimation and interpretation of the model, which we briefly discuss here.

\subsection{Observable quantities}

The two main observables in COVID-19 data are

- the cumulative number of reported cases; and

- the cumulative number of COVID-19 fatalities $D_{t}$.

Of the two, fatalities are generally considered more reliable, as deaths are nearly always reported, while identification of cases requires testing or self-reporting. We thus identify the observed number of fatalities with the state variable $D_{t}$.

In absence of widespread testing, only a fraction $\pi$ of cases are reported. This fraction may change with time due to testing campaign $4^{4}$. We therefore cannot assume the number of infectious cases to be directly observed: rather, we estimate it from the fatality count $D_{t}$ (see also Jombart et al. [26]).

Let $C_{t}$ be the cumulative number of (symptomatic) infectious cases. Assuming that

- the daily number $r(t)$ of reported cases is a fraction $\pi(t)$ of new cases, that is

$$
r(t)=\pi(t)\left(C_{t+1}-C_{t}\right)
$$

- deaths occur on average $T$ days after detection;

we obtain that the daily fatality count is proportional to the lagged number of new cases,

$$
D_{t+T+1}-D_{t} \simeq \bar{f}\left(C_{t+1}-C_{t}\right)=\frac{\bar{f}}{\pi(t)} r(t),
$$

where $\bar{f}$ is the (average) infection fatality rate. We use these relations to obtain an estimate for the cumulative number $C_{t}$ of symptomatic infections and the reporting ratio $\pi(t)$.

Using Eq. 4.2 we estimate the average delay $T$ between case reporting and death by identifying the lag $T$ which maximizes the correlation between the $D_{t+T+1}-D_{t}$ and $r(t)$. Using an average fatality rate of $\bar{f}=0.9 \%$ for the UK as in Ferguson et al. [22] (see discussion in Section 3.3), we estimate the reporting probability to be

$$
\widehat{\pi(t)}=\frac{\bar{f} r(t)}{D_{t+T+1}-D_{t}}
$$

which implies that the total number of cases in England is more than 20 times the reported number. As shown in Figure 6, prior to June 2020 this reporting ratio was around $\widehat{\pi(t)}=$

\footnotetext{
${ }^{4}$ See https: //ourworldindata.org/coronavirus-testing.
} 
$4.5 \%$; with the subsequent increase in testing, the estimated reporting ratio has steadily increased to more than $20 \%$ in November 2020.

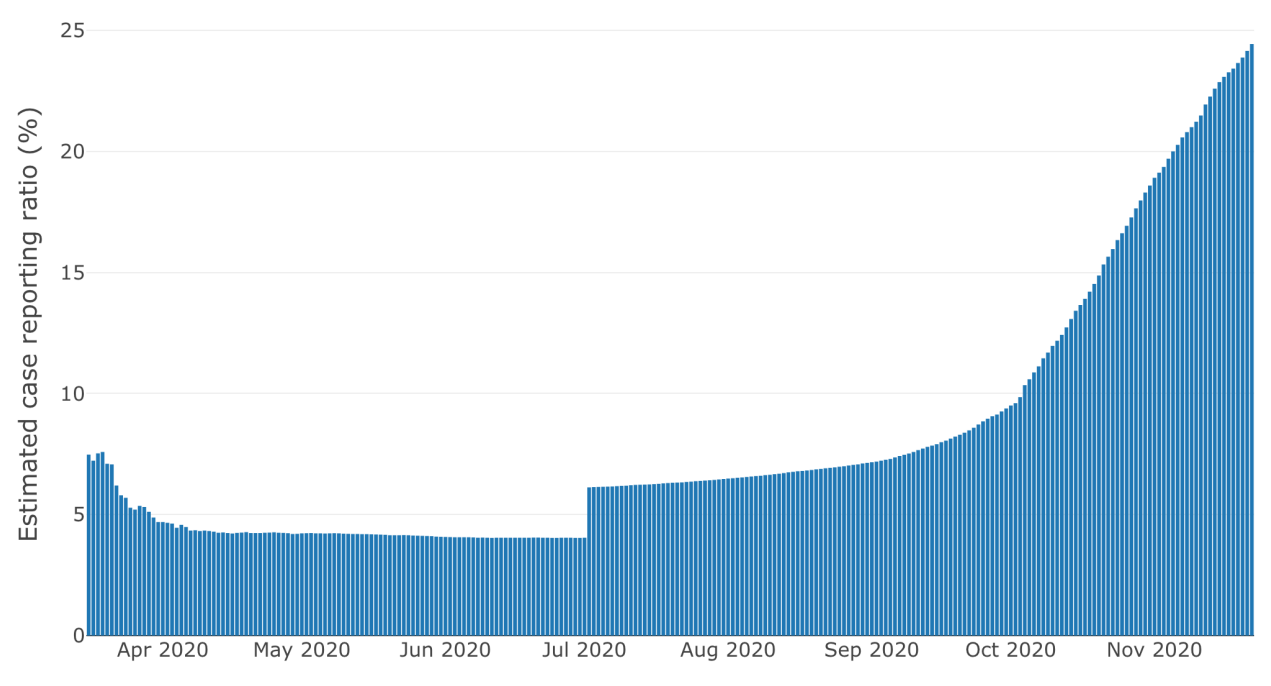

Figure 6: Estimate of case reporting ratio $\pi(t)$ based on a comparison of fatalities and reported cases.

\subsection{Implications of partial observability}

A key issue in epidemic control is the availability of reliable indicators for the intensity of an ongoing epidemic. Public health authorities have communicated the daily number of reported cases and fatalities, and these have served as inputs for policy planning.

An important corollary of the above discussion is that, given the combination of random factors affecting dynamics and the considerable uncertainty on the actual number of new infections, it is perfectly possible to observe a run of many consecutive days without new reported cases while in fact the actual number of infections is on the rise. 


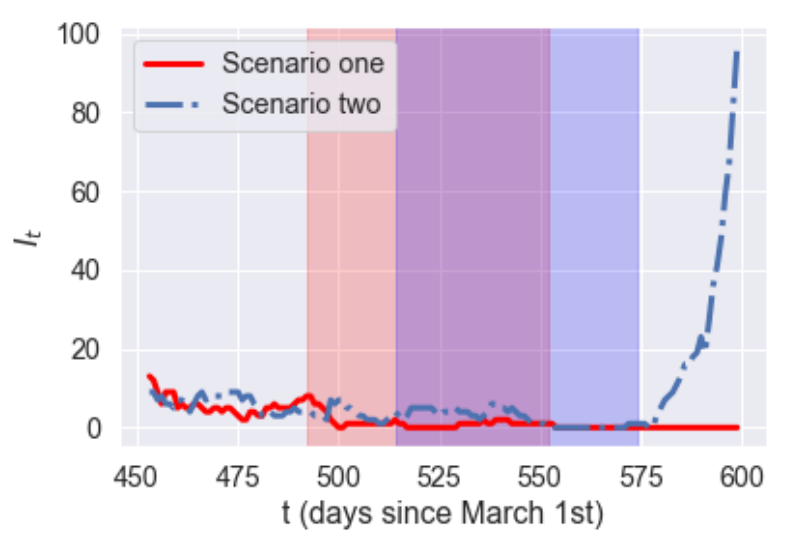

(a) Number of infected $I_{t}$.

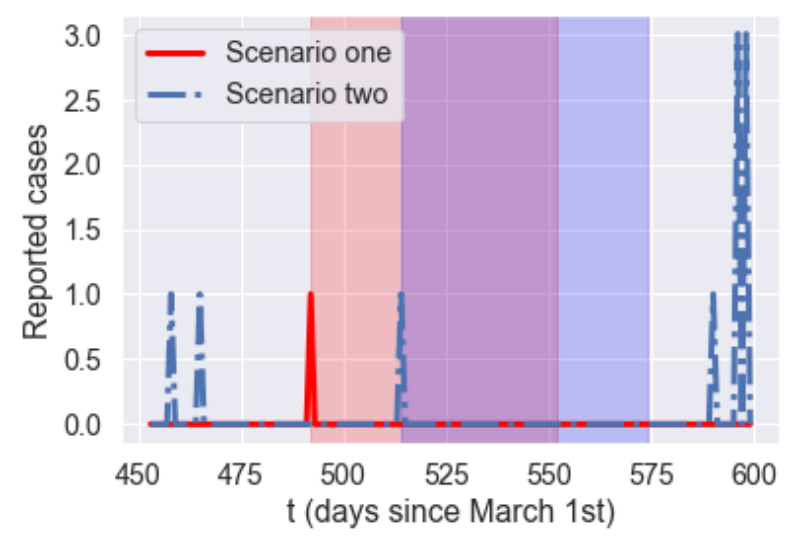

(b) Reported cases.

Figure 7: Example of latent progression of the epidemic with zero reported case for 60 consecutive days (red shaded area for scenario 1 and blue shaded area for scenario 2). Reporting probability is $\pi=4.5 \%$.

Figure 7 shows an example of scenario in our model where, for 60 consecutive days, although a small number of (symptomatic and asymptomatic) cases appear, due to the low detection probability $(\pi=4.5 \%)$, none of them is reported. Nevertheless, after a run of 60 days without any reported cases (blue shaded area in Figure 7), which may prompt public health authorities to lower their guard, the epidemic takes off again. Figure 7 displays in fact two sample paths with the same initial conditions, which differ only through the stochasticity of the dynamics. The fact that the break-out occurs only in one of the two scenarios (in blue) but no in the other illustrates how random flare-ups may originate from a small group of undetected cases.

Figure 8a shows the probability of observing a second peak in infections when social distancing measures are lifted after no reported cases for $L$ consecutive days. This probability is estimated using 500 simulated paths from 2.3. It is striking to observe that, even after 60 days with no reported cases, the probability of observing a resurgence of the epidemic is around 40\%. Figure 8a (blue dashed line) shows the same probability conditional on observing no fatalities for $L$ consecutive days.

These observations point to the importance of broader testing: as shown in Figure 8b. an increase in the probability $\pi$ of detecting new cases leads to a strong decrease in the probability of misdiagnosing the end of the epidemic, as in the scenario described above. 

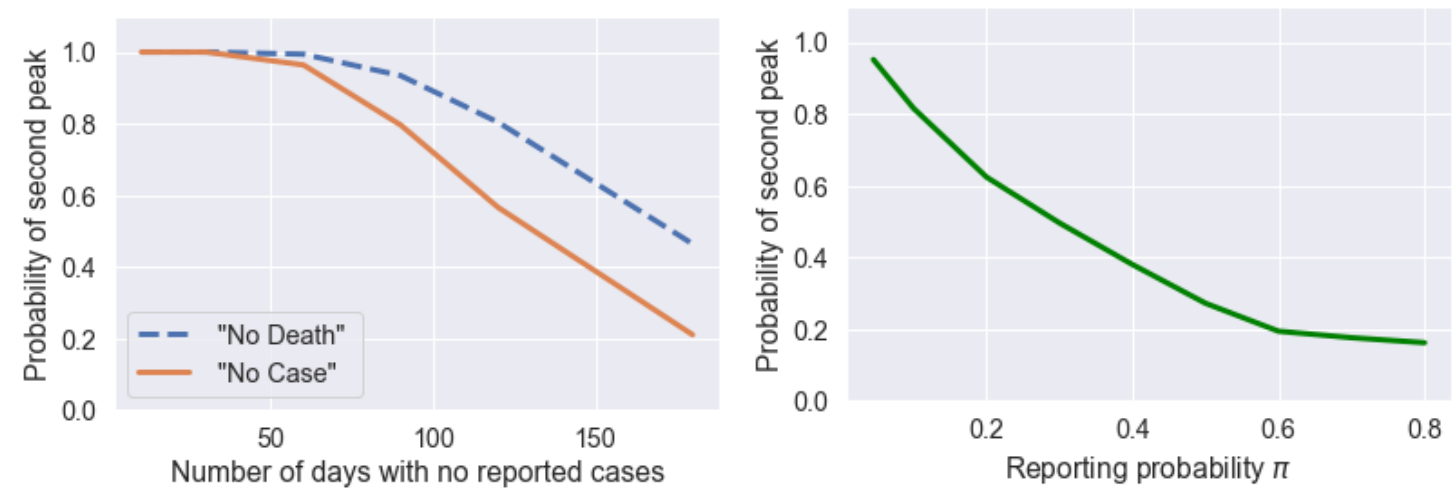

(a) Probability of having a second peak in (b) Probability of having a second peak in infections after no reported cases (solid line) infections following 60 consecutive days with and no fatalities (dashed line) for $L$ consec- no reported cases, as a function of reporting utive days (low symptomatic ratios). probability $\pi$.

Figure 8: Probability of observing a second peak after a period with no cases reported.

\section{Comparative analysis of epidemic control policies}

\subsection{Confinement followed by social distancing}

We first consider the impact of a national 'lockdown' followed by social distancing, which reflects the situation in the UK between March 2020 and August 2020. We examine in particular the impact of a lockdown duration $T$ and the level of social distancing after lockdown on the number of fatalities and the associated social cost. To do so, we parameterise the contact matrix as

$$
\sigma^{r}(t)= \begin{cases}l_{r} \sigma & \text { for } t_{0} \leq t \leq t_{0}+T \quad \text { (lockdown) } \\ \left((1-m) l_{r}+m d_{r}\right) \sigma & \text { for } t>t_{0}+T \quad \text { (after lockdown) }\end{cases}
$$

where $l_{r}$ measures the level of social distancing under lockdown, as estimated from observations for the period from Mar 23 to May 31, and the parameter $m \in[0,1]$ measures the level of compliance with social distancing measures. A value of $m$ close to zero indicates a level of social contact similar to lockdown, while $m=1$ corresponds to normal levels of social contact.

The origin date $t=0$ corresponds to March 1, 2020. All scenario simulations include a lockdown starting at $t_{0}=$ March 23, 2020. We consider a range $105 \leq T \leq 335$ for the lockdown duration and $0.2 \leq m \leq 1$ for post-lockdown social distancing levels. Note that the actual duration of the first lockdown in England corresponded to $T=105$.

As shown in Figure 9a, the level of social distancing after the confinement period is observed to be more important (Figure 9b) than the length of the confinement period (Figure 9a). This is consistent with the findings in Lipton and Lopez de Prado [34]. Smaller values of $m$, associated with stricter social distancing, lead to a lower of fatalities but for at an increased social cost (Figure 9b). On the other hand, the lengthening of 
the lockdown duration $T$, while significantly increasing the associated social cost, does not result in a significant reduction in the number of fatalities, especially if social distancing is not respected after lockdown.

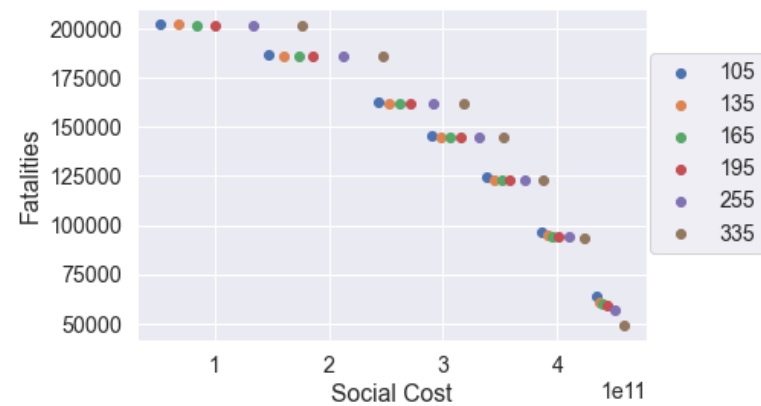

(a) Impact of lockdown duration $T$.

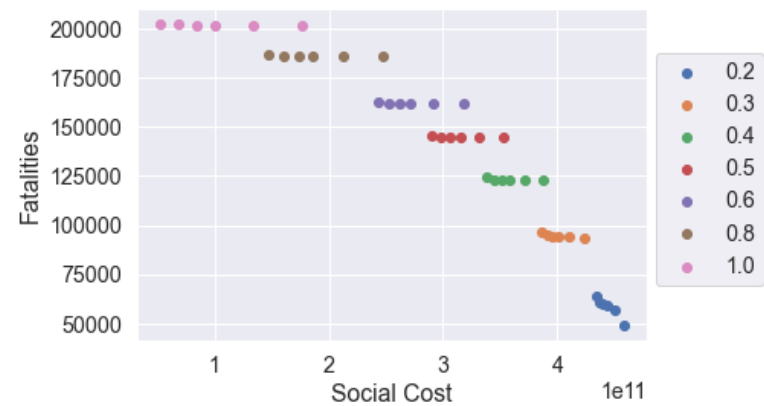

(b) Impact of compliance level $m$.

Figure 9: Fatalities against social cost for different $T$ and $m$ values. (Results for low symptomatic ratios).

Figure 9 also shows that some of these policies are inefficient, in the sense that we can reduce fatalities and the social cost simultaneously by shortening the lockdown period or by relaxing social distancing constraints, as shown in Figure 10.

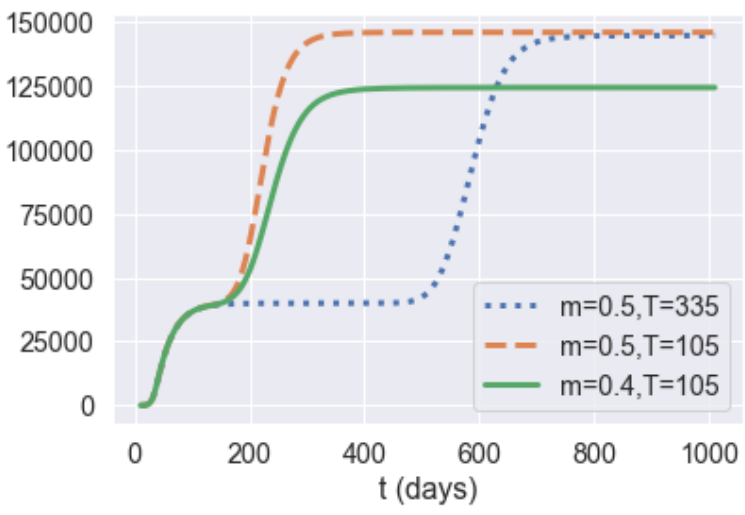

(a) Cumulative fatalities in England.

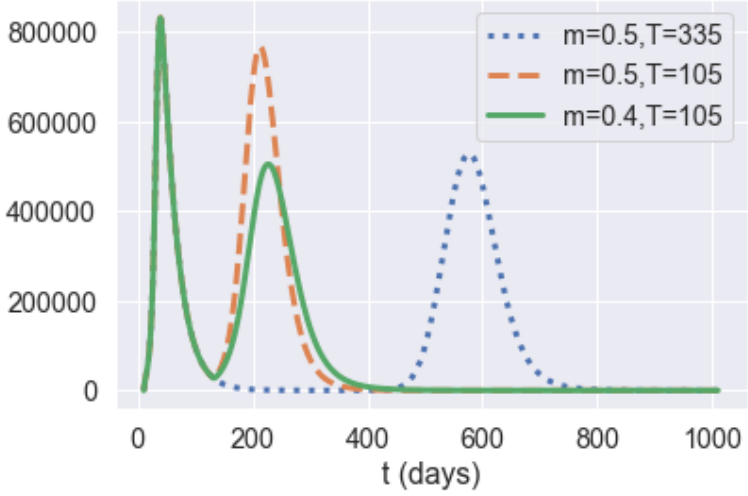

(b) Dynamics of $I_{t}$ in England.

Figure 10: Comparison of three policies: blue dotted line: $m=0.5$ and $T=335$; orange dashed line: $m=0.5$ and $T=105$; green solid line: $m=0.4$ and $T_{2}=105$. Average across 50 simulated scenarios.

\begin{tabular}{|l||l|l|l||}
\hline Policy & Blue dotted: & Orange dash: & Green solid: \\
& $m=0.5, T=335$ & $m=0.5, T=105$ & $m=0.4, T=105$ \\
\hline \hline Social cost $\left(\times 10^{11}\right)$ & 3.5 & 2.9 & 3.4 \\
Projected fatalities & 144,600 & 146,000 & 124,400 \\
\hline \hline
\end{tabular}

Table 7: Outcomes for policies represented in Figure 10 
By comparing the orange and blue plots, which represent the same post-lockdown compliance level $(m=0.5)$, we observe that extending the lockdown duration increases social cost without reducing the total number of fatalities. On the other hand, comparing the orange and green plots, which correspond to the same lockdown duration of $T=105$ days, shows that moving the compliance level from $m=0.5$ to $m=0.4$ reduces the second peak amplitude by $35 \%$ and fatalities by $13.9 \%$.

Impact of parameter uncertainty The above results are highly sensitive to the value of the symptomatic ratios which, as noted in Section 3, are highly uncertain (see Table 33. Figure 11 shows the policy outcomes for low versus high symptomatic ratios across different compliance levels and lockdown duration. As observed in this figure, while the overall pattern of the efficiency diagram is similar, the projected fatality levels shift considerably depending on the assumption on the symptomatic ratio: from 50,000-200,000 for low symptomatic ratios to $126,000-430,000$ for high symptomatic ratios.

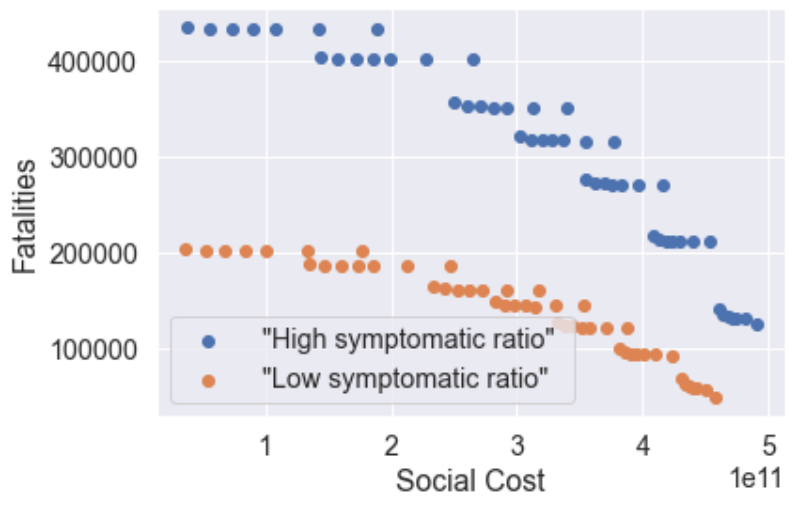

Figure 11: Trade-off between fatalities and social cost for a $T$-day lockdown followed by social distancing $(0.2 \leq m \leq 1,105 \leq T \leq 335)$ : low symptomatic ratio (orange) and high symptomatic ratio (blue).

Regional heterogeneity While the policies discussed here are applied uniformly across all regions, we observe a significant heterogeneity in mortality levels across regions, as well in terms of the timing and amplitude of a second peak tin infections. As shown in 12 , some regions exhibit mortality levels up to 4 times higher than others. This huge disparity in mortality rates cannot be explained by demographic differences alone, which are much less pronounced: more important seem to be the differences in social contact patterns, as illustrated in Figure 2. Indeed, as shown in Figure 13a, there is a positive correlation (above 40\%) between regional COVID-19 mortality and the intensity of social contact as measured by the parameter $d_{r}$, defined in Section 3.3 . Figure $13 \mathrm{~b}$ shows that this heterogeneity is also reflected in the timing and amplitude of second peaks. 


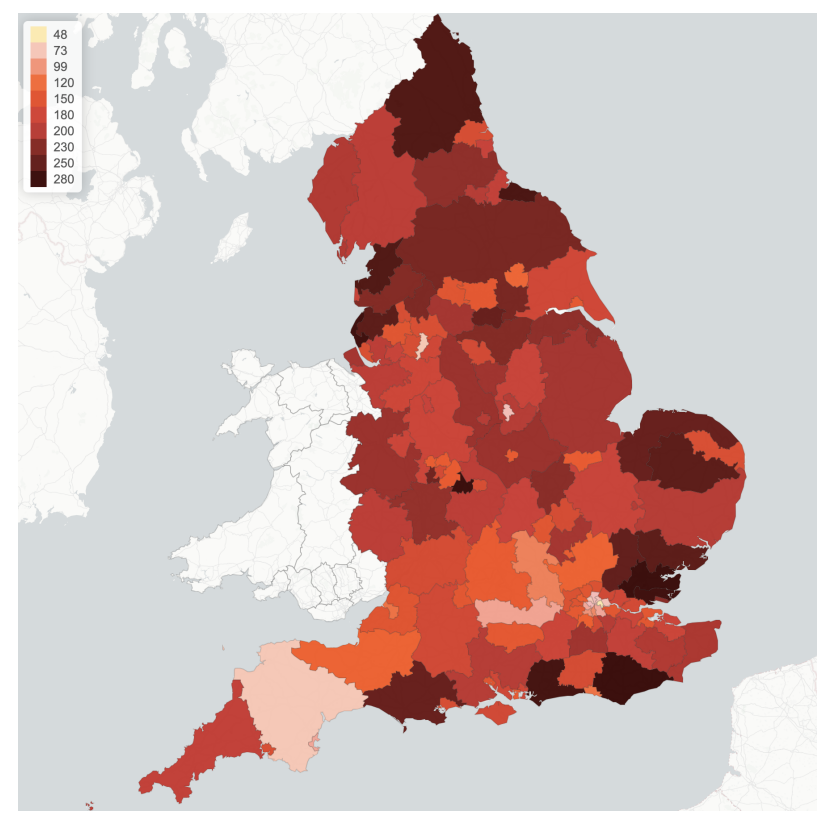

Figure 12: Lockdown of 105 days followed by social distancing $(m=0.3)$ : regional mortality per 100,000 inhabitants.

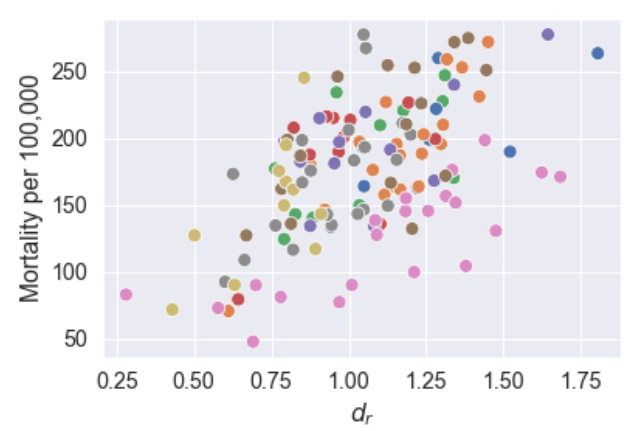

(a) Level of social contact $\left(d_{r}\right)$ against mortality (per 100,000 inhabitants).

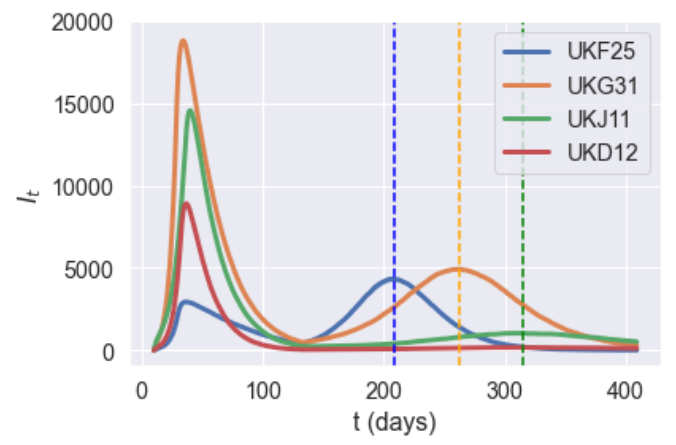

(b) Regional dynamics of symptomatic infections $\left(I_{t}\right)$ : North NorthamptonBerkshire (UKJ11) and East Cumbria (UKD12). Dotted lines denote the secondpeak times.

Figure 13: Regional outcomes for lockdown of 105 days followed by social distancing $(m=$ $0.3)$. 


\section{$5.2 \quad$ Targeted policies}

We now consider the impact of social distancing measures targeting particular age groups or environments (school, work, etc.) following a lockdown of duration $T$, by setting

$$
\sigma_{i j}^{r}(t)= \begin{cases}l_{r} \sigma & \text { for } t_{0} \leq t \leq t_{0}+T \quad \text { (lockdown) } \\ \sigma_{i j}^{r, H}+u_{i j}^{S} \sigma_{i j}^{r, S}+u_{i j}^{W} \sigma_{i j}^{r, W}+u_{i j}^{O} \sigma_{i j}^{r, O} & \text { for } t>t_{0}+T .\end{cases}
$$

We consider different targeted measures after a lockdown period of $T=105$ days (the actual duration of the lockdown in England): school closure, shielding of elderly populations and workplace restrictions, restrictions on social gatherings and combinations thereof. Note that there is no control over the social contacts at home.

School closures Although most of the infected population in age group 1 is asymptomatic, they may in turn infect the population in age groups 3 and 4 who are more likely to develop symptoms. School closure corresponds to $u^{S}=0$, school reopening with social distancing correspond to $u^{S}=0.5$, and school reopening without social distancing correspond to $u^{S}=1$.

Shielding The high infection fatality rates among elderly populations (age groups 3 and 4) have naturally lead to consider shielding policies for these populations. We model this as a reduction in social contacts of age groups 3 and 4 to the level observed under lockdown:

$$
\sigma_{i, j}^{r}(t)=l_{r}\left(\sigma_{i, j}^{H}+\sigma_{i, j}^{S}+\sigma_{i, j}^{W}+\sigma_{i, j}^{O}\right) \quad \text { if } \quad i \in\{3,4\} \quad \text { or } \quad j \in\{3,4\} .
$$

Workplace restrictions We model the impact of a restricted return to work after confinement by assuming different proportion of workforce return after the lockdown period by choosing

$$
0.2<u^{W}<1 \text { for } t>t_{0}+T,
$$

the lower bound $u^{W}=0.2$ corresponding to restricting workplace return to 'essential workers', as discussed in Section 3.5. Since workplace restrictions have an effect on commuting, such measures also have an impact on the inter-regional mobility matrix

$$
C_{t}=u^{W}(t) C_{0}+\left(1-u^{W}(t)\right) I,
$$

where $C_{0}\left(r, r^{\prime}\right)$ is the baseline mobility matrix defined in (3.1).

Restrictions on social gatherings Although social activities, such as gatherings at pubs or sports events, may aggravate the contagion of COVID-19, keeping certain levels of social activities is important to the economic recovery and the well-being of individuals. The parameter $u^{O}$ measures the fraction of social gatherings: during the lockdown this fraction was estimated to be as low as $20 \%$ (see Section 3.5). In what follows, we consider $u^{O} \in[0.3,1.0]$ after the period of lockdown. 


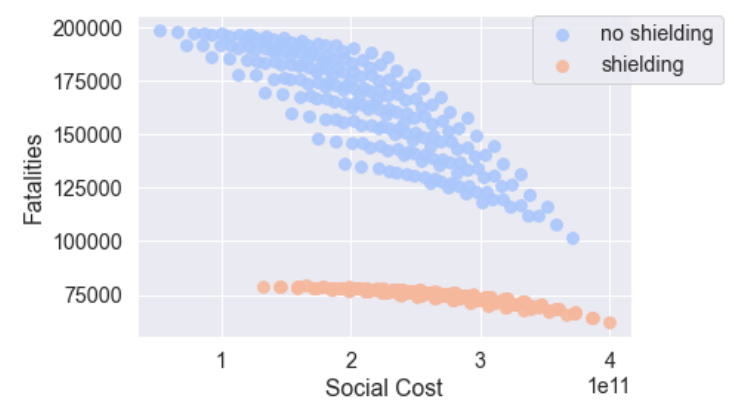

(a) Impact of the shielding measure for senior citizens.

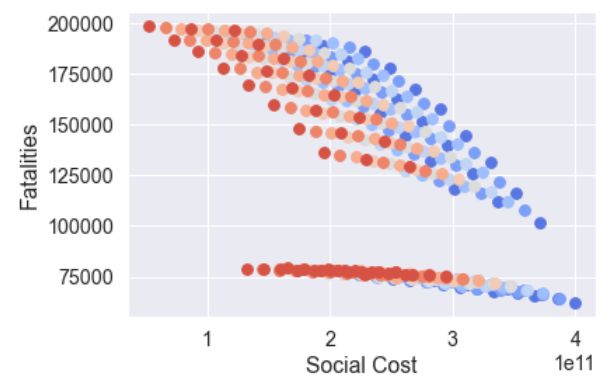

(c) Social distancing at work: impact of the parameter $u^{W}$.

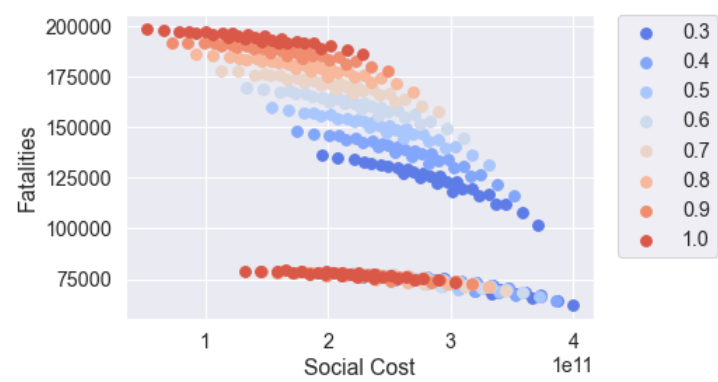

(b) Social distancing outside work and school: impact of the parameter $u^{O}$.

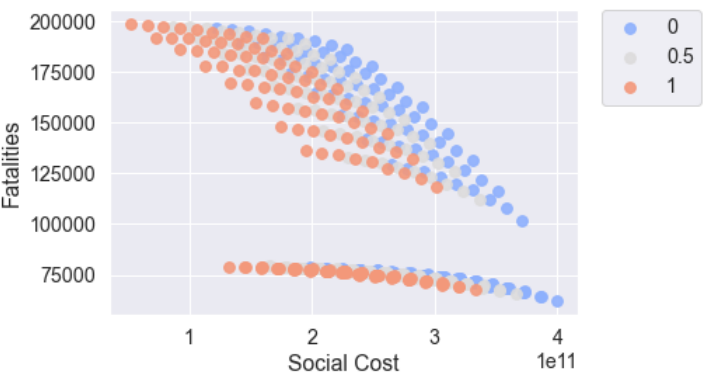

(d) Social distancing at school: impact of the parameter $u^{S}$.

Figure 14: Efficiency plot of social cost against projected fatalities for the shielding measure and various values of $u^{S}, u^{W}$, and $u^{O}\left(u^{H}=1\right.$ and $\left.T=105\right)$.

\subsubsection{Pubs and schools}

\begin{tabular}{|l|l|l|l|}
\hline & $\begin{array}{l}\text { School } \\
\text { closure } \\
u^{S}=0\end{array}$ & $\begin{array}{l}\text { Social distancing } \\
\text { at school } \\
u^{S}=0.5\end{array}$ & $\begin{array}{l}\text { Normal school } \\
\text { regime } \\
u^{S}=1.0\end{array}$ \\
\hline \hline Social cost $\left(10^{11}\right)$ & 2.2 & 1.9 & 1.5 \\
Projected fatalities & 153,900 & 157,000 & 159,300 \\
\hline
\end{tabular}

Table 8: Impact of school closures and social distancing at schools: outcomes averaged across 50 simulated scenarios, $u^{H}=u^{W}=1, u^{O}=0.5$.

Table 8 show the impact of school closures and social distancing at schools on projected fatalities and social contacts. Reopening of schools, while reducing significantly the social cost, does not seem to lead to a significant increase in fatalities. 


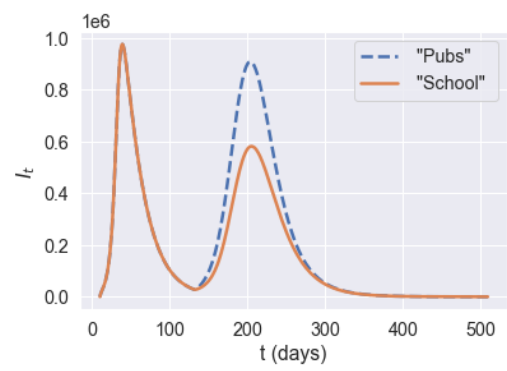

(a) Infections in England.

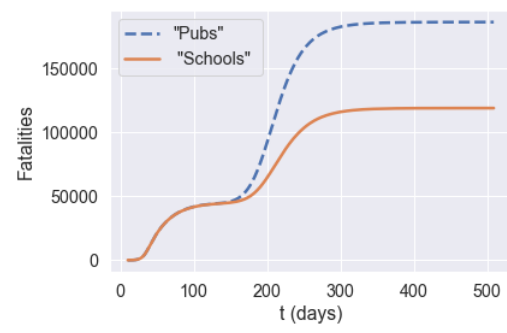

(b) Fatalities in England.

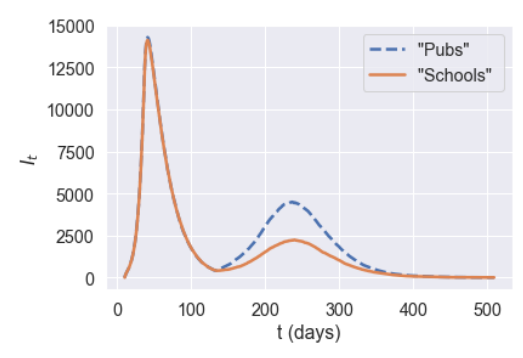

(c) Infections: Oxfordshire (UKJ14).

Figure 15: 'Open pubs' versus 'open schools' policy.

We compare two post-confinement policies, one (labeled as 'schools') consisting in leaving schools open while social gatherings are restricted $\left(u^{S}=1, u^{O}=0.2\right)$, and the other (labeled as 'pubs') consisting in closing schools while not restricting social gatherings $\left(u^{S}=0, u^{O}=1\right)$. The social cost for the 'Pubs' policy is 2.3 , while the cost for the 'Schools' policy is 3.0. However, as shown in Figure 15, the 'open school' policy leads to $35 \%$ fewer fatalities compared to the 'open pubs' policy.

\subsubsection{Shielding of senior citizens}

We have examined the impact of shielding in isolation and also in combination with other measures such as school closure and social distancing.

As shown in Figure 14a, whether applied in isolation or in combination with other measures, shielding of elderly populations is by far the most effective measure for reducing the number of fatalities. As clearly shown in Figure 14a, regardless of the trade-off between social cost and health outcome, a policy which neglects shielding of the elderly is not efficient and its outcomes can always be improved through shielding measures.

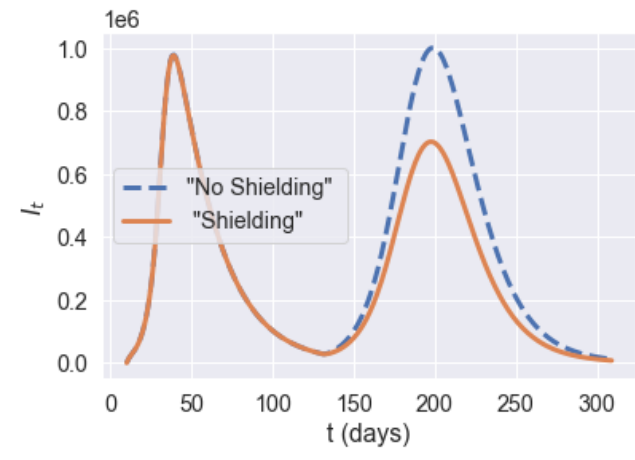

(a) Symptomatic infections in England.

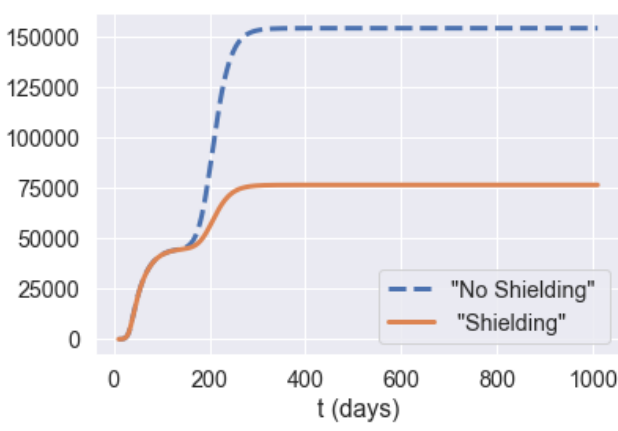

(b) Fatalities in England.

Figure 16: Comparison of policies with and without shielding in place, $u=(1,0.0,1.0,0.5)$. Blue: no shielding; orange: shielding in place. 
Impact of shielding on policy efficiency For policies without shielding, the level of social gatherings, $u^{O}$, is the leading factor to determine the efficiency frontier. In Figures 17a, the efficiency frontier contains two classes of policies: 'School and Work' and 'No Pubs'.

- 'School and Work' policies, which do not include any restrictions on school or work $\left(u^{S}=1, u^{W}=1\right)$ but varying level of restrictions on social gatherings $\left(0.3 \leq u^{O} \leq 1\right)$. Within this class of policies, different level of social gatherings lead to very different outcome of fatalities, as illustrated in Figure 17a.

- 'No Pubs' policies, where social gatherings outside school and work are restricted $\left(u^{O}=0.3\right)$, with different levels of social distancing $u^{S} \in\{0,0.5,1\} u^{W} \in[0.2,1]$ at school and work.

However, as observed in Figure $17 \mathrm{~b}$, these policies are not efficient when shielding measures are put in place for the elderly.

Under shielding, the spectrum of efficient policies is parameterised by the fraction $u^{W}$ of the workforce returning to work. As shown in Figure $17 \mathrm{c}$, we can distinguish two classes of efficient policies under shielding:

- 'School and Pubs', consisting of policies without restrictions on schools or social gatherings $\left(u^{S}=1, u^{O}=1\right)$ and different levels $u^{W}$ of restrictions on workplace gatherings.

- 'Restricted Work' policies, under which only 'essential' workers are allowed on-site work $\left(u^{W}=0.2\right)$, with either

(i) no school restrictions $\left(u^{S}=1\right)$ and different levels of restrictions on social gatherings $\left(0.2 \leq u^{O} \leq 1\right)$ or

(ii) restrictions on social gatherings $\left(u^{O}=0.3\right.$, that is 'no pubs') and different levels of social distancing in school $\left(0 \leq u^{S} \leq 1\right)$.

As Figure 17d illustrates, 'School and Pubs' and 'Restricted Work' policies are not efficient without shielding.

In absence of shielding, social gatherings seem to be the main vector for contagion. When shielding measures are put in place, the social contacts associated with the elderly are reduced to the same level as under lockdown; in this case, contacts at work become the main vector of contagion. 

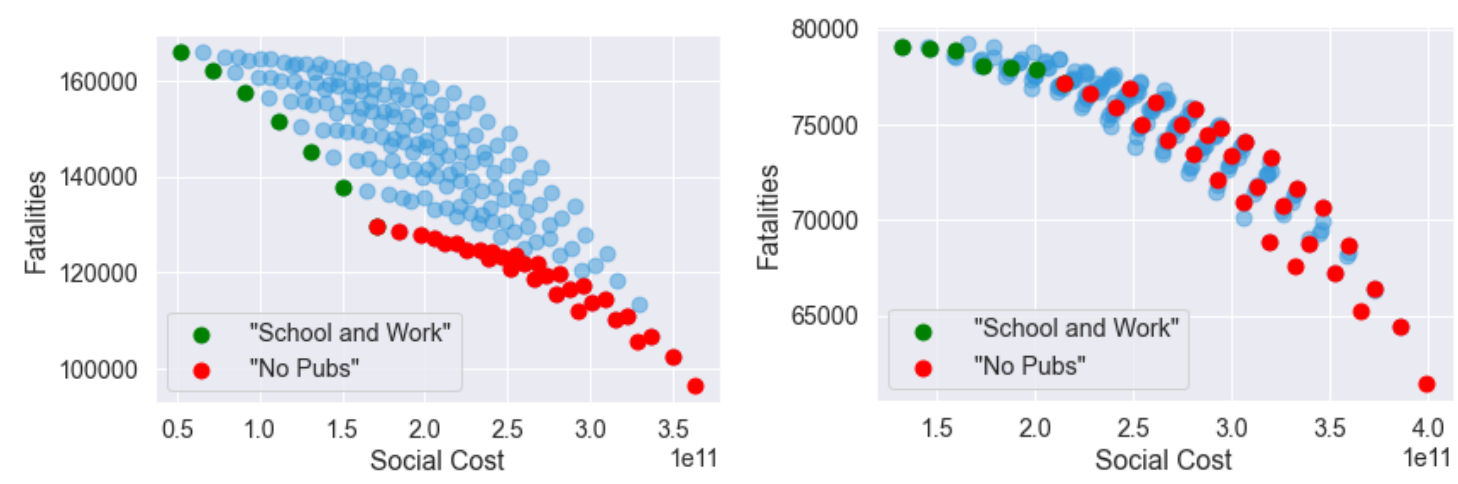

(a) Efficient policies without shielding: (b) Policies 'School and Work' and 'No 'School and Work' and 'No Pubs'.

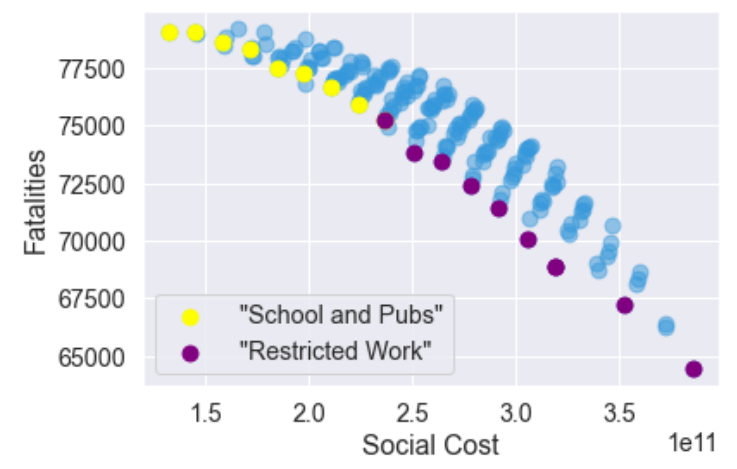

Pubs' are not efficient when shielding is applied.

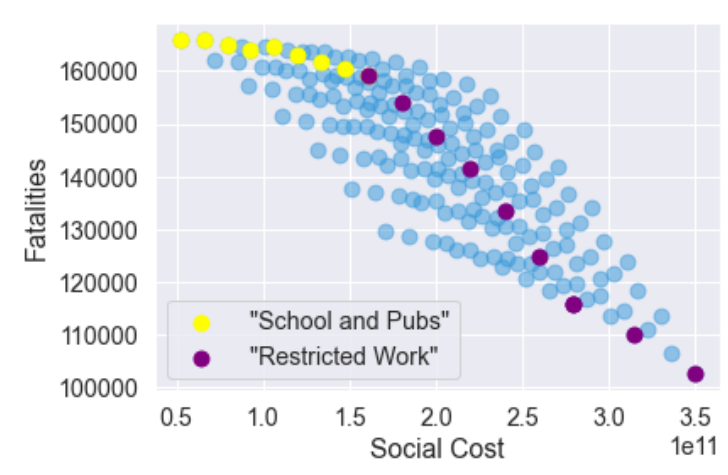

(c) Efficient policies with shielding: 'School (d) Policies 'School and Pubs' and 'No work' and Work' and 'No work'.

are not efficient when shielding is removed.

Figure 17: Impact of shielding on the efficiency frontier.

\section{Adaptive mitigation policies}

We now consider adaptive mitigation policies, in which the daily number of (national or regional) reported cases is used as a trigger for social distancing measures. Such policies have been recently implemented, in the UK and elsewhere, at a local or national level with various degrees of success. We distinguish centralised policies, based on monitoring of national case numbers, from decentralised policies where monitoring and implementation of measures are done at the level of (NUTS-3) regions.

\subsection{Country-wide restrictions}

We first consider centralised policies which monitor the number of daily reported cases at country level. Whenever the number of daily reported cases (per 100,000 inhabitants) exceeds a threshold $B_{\text {on }}$, confinement measures are imposed for a minimum of $L$ days, until the number of daily reported cases falls below the threshold $B_{\text {off }}<B_{\text {on }}$. Outside these lockdown periods, we assume social distancing is in place with a compliance level $m$; 
we use a default value of $m=0.5$.

This policy is implemented after the initial lockdown (that is, after July 4, 2020). In terms of the social contact matrix, we have, for $t>t_{0}+T$,

$$
\begin{cases}\sigma^{r}(t)=\left((1-m) l_{r}+m d_{r}\right) \sigma, \text { and } i_{s}=0, & \text { if }\left(C_{t} \leq \frac{N}{100,000} B_{\text {off }} \text { and } \Pi_{s=t-L}^{t-1} i_{s}=1\right) ; \\ \sigma^{r}(t)=l_{r} \times \sigma, \text { and } i_{t}=1, & \text { or }\left(C_{t} \leq \frac{N}{100,000} B_{\text {on }} \text { and } i_{t-1}=0\right) ; \\ \text { if } C_{t}>\frac{N}{100,000} B_{\text {on }} \text { or } \Pi_{s=t-L}^{t-1} i_{s}=1 .\end{cases}
$$

Here $T=105, i_{t}$ is the indicator of whether lockdown is applied on day $t$ and $C_{t}$ is the daily reported cases in England on day $t . \Pi_{s=t-L}^{t-1} i_{s}=1$ if lockdown has been applied for $L$ consecutive days during the period $[t-L, t-1]$.

We simulate the dynamics with various choices of $B_{\text {off }}$ and $B_{\text {on }}$ :

- $B_{\text {on }} \in\{2,4,6,8,10\}$ (daily reported cases per 100,000 inhabitants); and

- $B_{\text {off }}=0.2 B_{\text {on }}, B_{\text {off }}=0.4 B_{\text {on }}$ or $B_{\text {off }}=0.8 B_{\text {on }}$.

We assume that once a lockdown is triggered it lasts a minimum of $L=7$ days and that, once lockdown is removed, individuals continue to observe social distancing as measured by the parameter $m \in[0,1]$. Data on real-time mobility monitoring in the UK ${ }^{5}$ indicate mobility to be at $50 \%$ of normal level during the post-lockdown period, and thus we use $m=0.5$ as a default value.
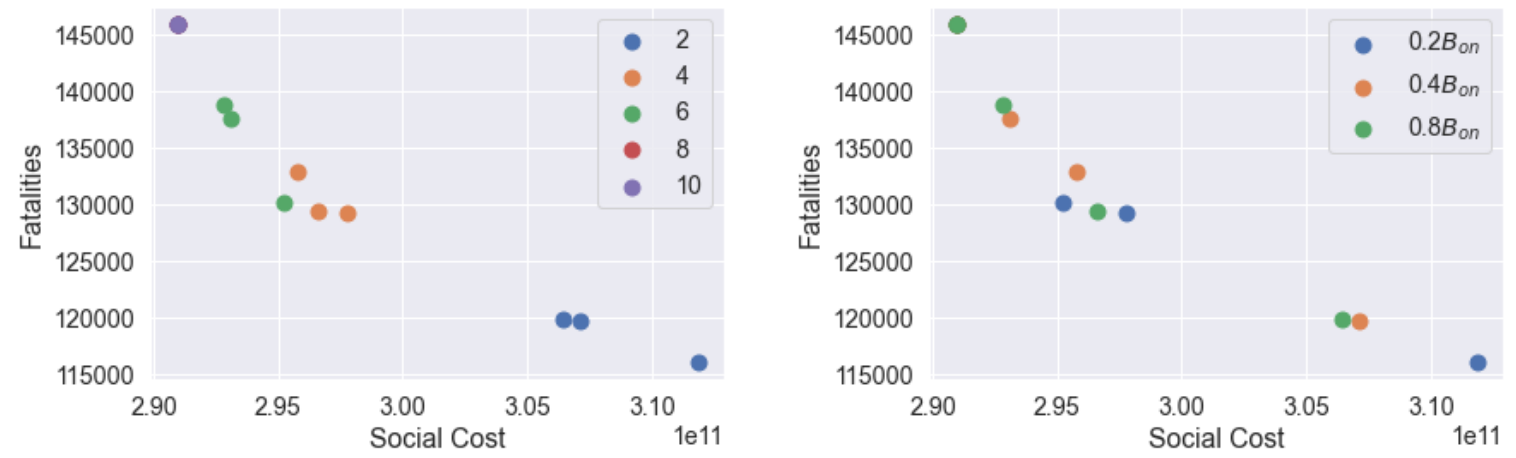

(a) Influence of the threshold $B_{\text {on }}$ to resume (b) Influence of the threshold $B_{\text {off }}$ to lift locklockdown. down.

Figure 18: Social cost against fatalities when $m=0.5$.

Example Figure 19 shows an example of such an adaptive policy, where lockdown is triggered when daily cases exceeds 2240 nationally, and maintained until the count of new daily cases drops to 896. In the scenario shown in Figure 19a, this results in two short lockdowns, totaling 19 days in all, which bring under control the national progression of the epidemic and avoid a 'second peak' at national level. However, as shown in Figure 19b, this policy is less successful at regional level, resulting in a regional outbreak in Leicester.

\footnotetext{
${ }^{5}$ See https: //www. oxford-covid-19.com/
} 


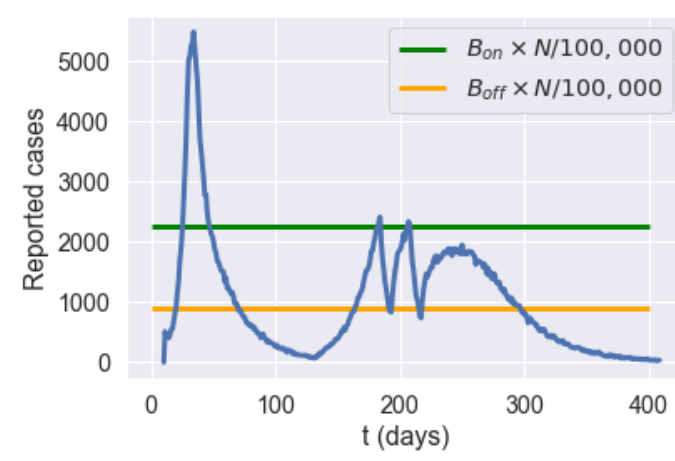

(a) Daily reported cases in England.

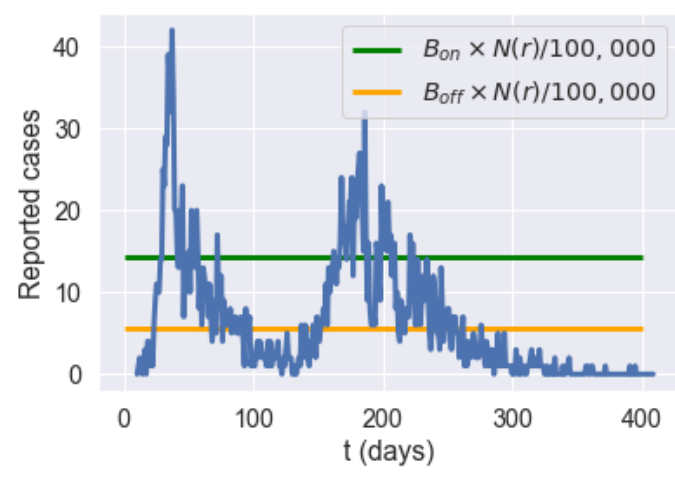

(b) Daily reported cases in Leicester.

Figure 19: Simulation of reported cases in England and Leicester under a centralised triggering policy with $B_{\text {on }}=4, B_{\text {off }}=0.4 \times R_{o n}, m=0.5$ and no shielding.

Impact of the triggering threshold $B_{\text {on }}$ The trigger threshold $B_{\text {on }}$ has a significant impact on the efficiency of the policy. Smaller $B_{\text {on }}$ values correspond to more frequent lockdowns, leading to a larger social cost and fewer fatalities. Here we compare the impact of the triggering threshold $B_{\text {on }}$ when $m=0.5$ and $B_{\text {off }}=0.4 \times B_{\text {on }}$.

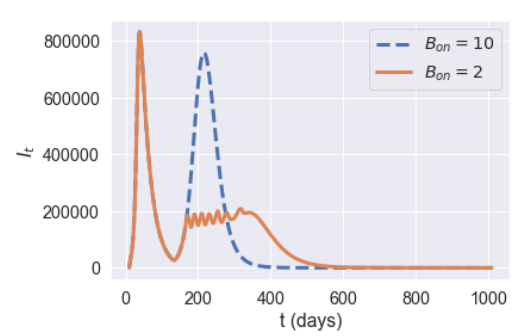

(a) Dynamics of $I_{t}$ in England.
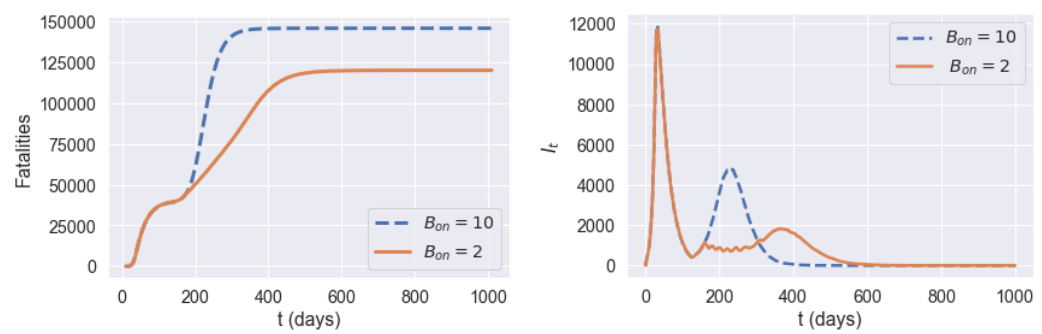

(b) Cumulative fatalities in (c) $I_{t}$ in Oxfordshire (UKJ14). England.

Figure 20: Comparison between triggering thresholds $B_{\text {on }}=10$ and $B_{\text {on }}=2$.

We observe in our simulations a second peak in $I_{t}$ for England when $B_{\text {on }}=10$, while we observe no second peak when $B_{\text {on }}=2$. When $B_{\text {on }}=2, I_{t}$ remains at level $2 \times 10^{5}$ with frequent interventions for 200 days and then decreases to zero. The social cost for policy $B_{\text {on }}=10$ and policy $B_{\text {on }}=2$ are 2.9 and 3.1, respectively. Policy $B_{\text {on }}=2$ have $18 \%$ fewer fatalities compared to policy $B_{\text {on }}=10$. Oxfordshire exhibits the same profile as England when $B_{\text {on }}=10$. However, the shape of $I_{t}$ is different for $B_{\text {on }}=2$ where Oxfordshire experiences a small outbreak around day 350 .

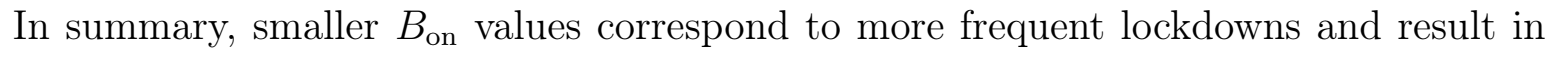
damping or elimination of the 'second peak'.

Increasing testing capacity To study the effect of an increased testing capacity, we assume wide testing is adopted such that the reporting probability is increased from $4.5 \%$ to a significantly higher level $(20 \%, 50 \%)$ on July 4, 2020 (see Figure 21). 


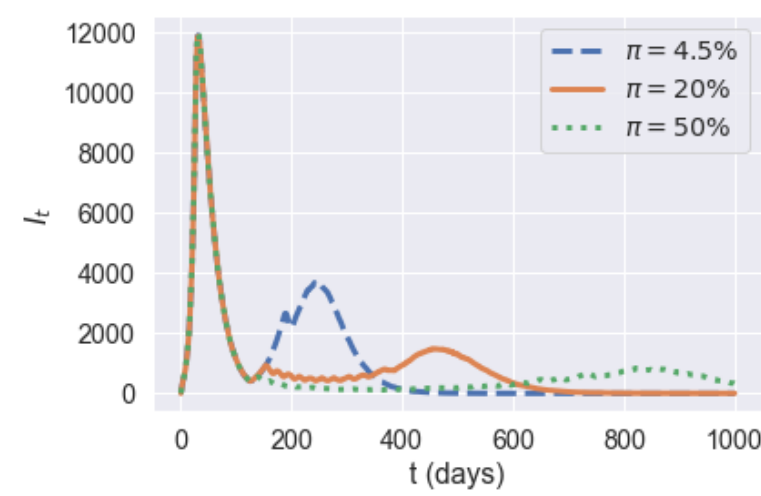

(a) Dynamics of $I_{t}$ in England.

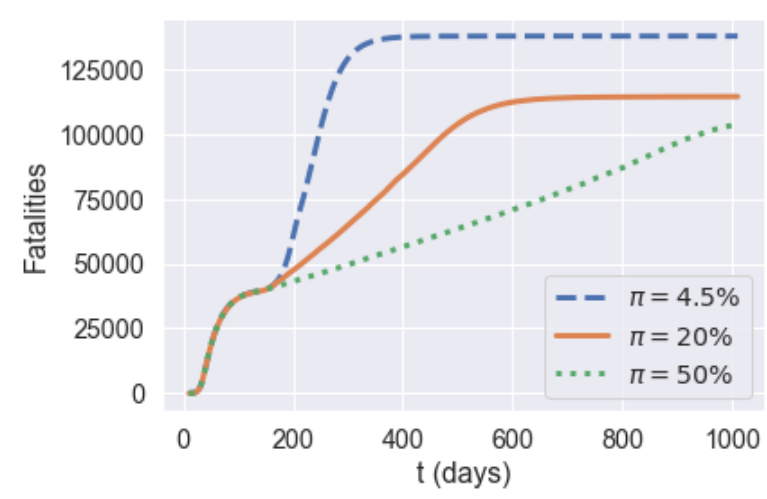

(b) Cumulative fatalities in England.

Figure 21: Reporting probabilities $\pi=4.5 \%$ (blue line) versus $\pi=20 \%$ (orange line) and $\pi=50 \%$ (green line). Policy: $B_{\text {on }}=6, B_{\text {off }}=0.2 B_{\text {on }}$, and $m=0.5$.

\begin{tabular}{|l||l|l|l||}
\hline Reporting prob. $\pi$ & $4.5 \%$ & $20 \%$ & $50 \%$ \\
\hline \hline Social Cost $\left(10^{11}\right)$ & 2.9 & 3.2 & 3.6 \\
Fatalities & 137,600 & 114,300 & 103,700 \\
\hline \hline
\end{tabular}

Table 9: Average social cost and fatalities for a given policy with different testing capacities (50 paths). Policy: $B_{\text {on }}=6, B_{\text {off }}=0.4 B_{\text {on }}$, and $m=0.5$.

By increasing the testing capacity, the observable quantity of daily reported cases becomes more consistent with the underlying dynamics of $I_{t}$. Compared to the policy with a reporting probability $\pi=4.5 \%$ throughout the reference period, we see that the dynamics of $I_{t}$ when $\pi=50 \%$ decrease to a small value rapidly. Increasing the testing capacity also implies a more efficient control and as a result leads to fewer fatalities.

Impact of demographic granularity Several studies on the impact of public health policies on COVID-19 dynamics have used less granular models with fewer age groups [1. To assess whether such coarse-graining may result in a loss of accuracy for the model projections, we have compared our present model, which has 16 age groups, with coarsegrained versions of the model in which all individuals in the 20-59 age range are grouped into 2 age groups (leading to a total of 5 age groups) or a single group (leading to 4 age groups). ${ }^{6}$ Parameters for the coarse-grained models are obtained as population-weighted averaged of the granular model.

Comparison of model projections, shown in Figure 22, indicate that the results are robust to changes in model granularity. Some quantitative differences may emerge when assessing the impact of targeted policies, but the overall dynamics of infections, cases and fatalities are rather insensitive to the demographic granularity.

${ }^{6}$ This model was implemented in a previous version of this paper: https://www.medrxiv.org/ content/10.1101/2020.08.26.20182477v2. 


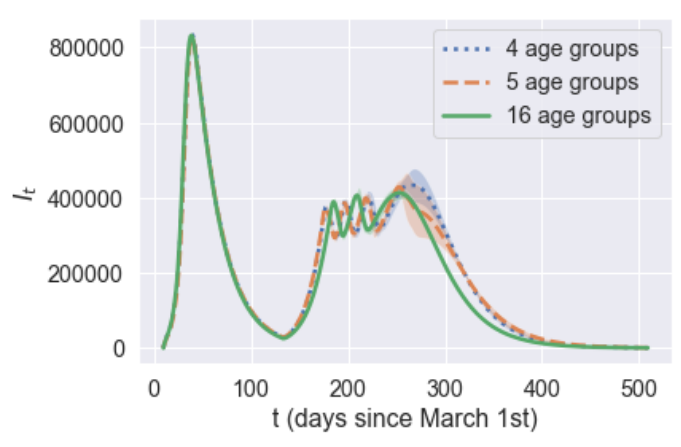

(a) Symptomatic infections $\left(I_{t}\right)$ in England.

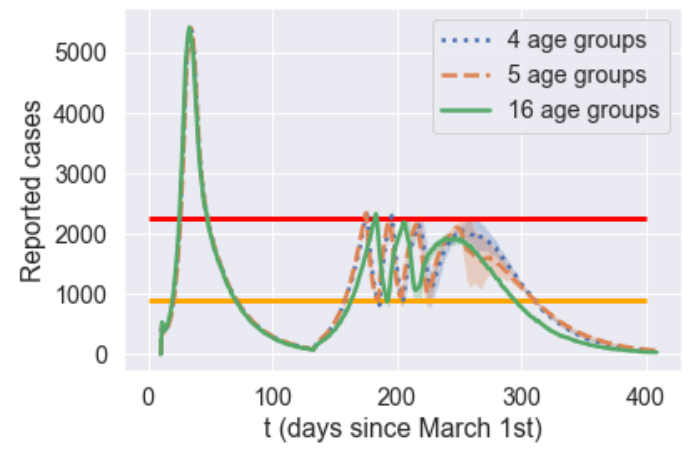

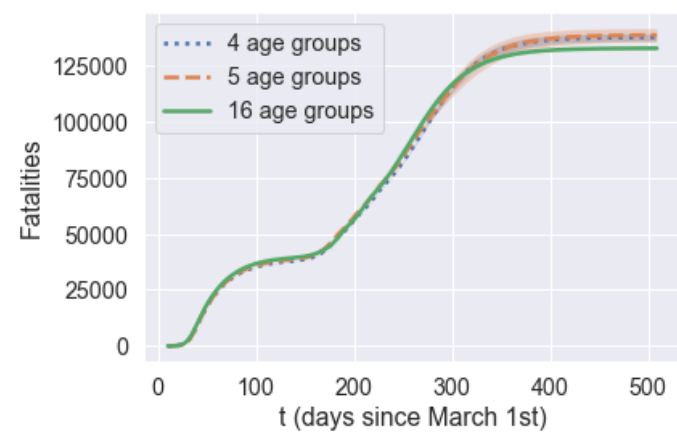

(b) Projected fatalities.

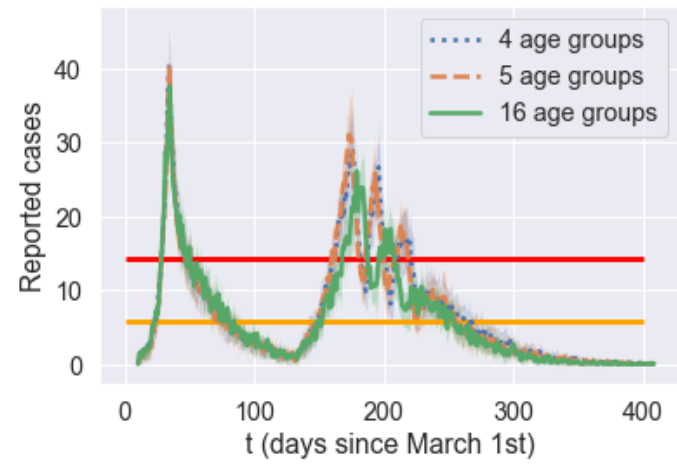

(c) Projections for reported cases: England. (d) Projections for reported cases: Leicester.

Figure 22: Impact of model granularity: projections for an adaptive policy with $R_{\text {on }}=4$, $R_{\text {off }}=0.4 \times R_{\text {on }}, m=0.5$ and no shielding.

\subsection{Decentralised policies}

We now consider a decentralised version of the above policies, based on monitoring of regional number of cases as triggers for regional confinement measures. In terms of the social contact matrices, we have, for $t>t_{0}+T$,

$$
\begin{cases}\sigma^{r}(t)=\left((1-m) l_{r}+m d_{r}\right) \sigma, \quad i_{t}^{r}=0, & \text { if }\left(C_{t}(r) \leq \frac{N(r)}{100,000} B_{\text {off }} \text { and } \Pi_{s=t-L}^{t-1} i_{s}^{r}=1\right) \\ \sigma^{r}(t)=l_{r} \times \sigma, \quad \text { and } \quad i_{t}^{r}=1, & \text { or }\left(C_{t}(r) \leq \frac{N(r)}{100,000} B_{\text {on }} \text { and } i_{t-1}^{r}=0\right) ; \\ & \text { if } C_{t}(r)>\frac{N(r)}{100000} B_{\text {on }} \text { or } \Pi_{s=t-L}^{t-1} i_{s}^{r}=1 .\end{cases}
$$

Here $i_{t}^{r}$ is the indicator of whether lockdown is applied in region $r$ on day $t$ and $C_{t}(r)$ is the daily number of cases reported in region $r$ on day $t$. The term $\Pi_{s=t-L}^{t-1} i_{s}^{r}$ is used to track if lockdown has been applied in region $r$ for $L$ consecutive days during $[t-L, t-1]$. We use the same values of $B_{\text {on }}$ and $B_{\text {off }}$ as in Section 6.1. 

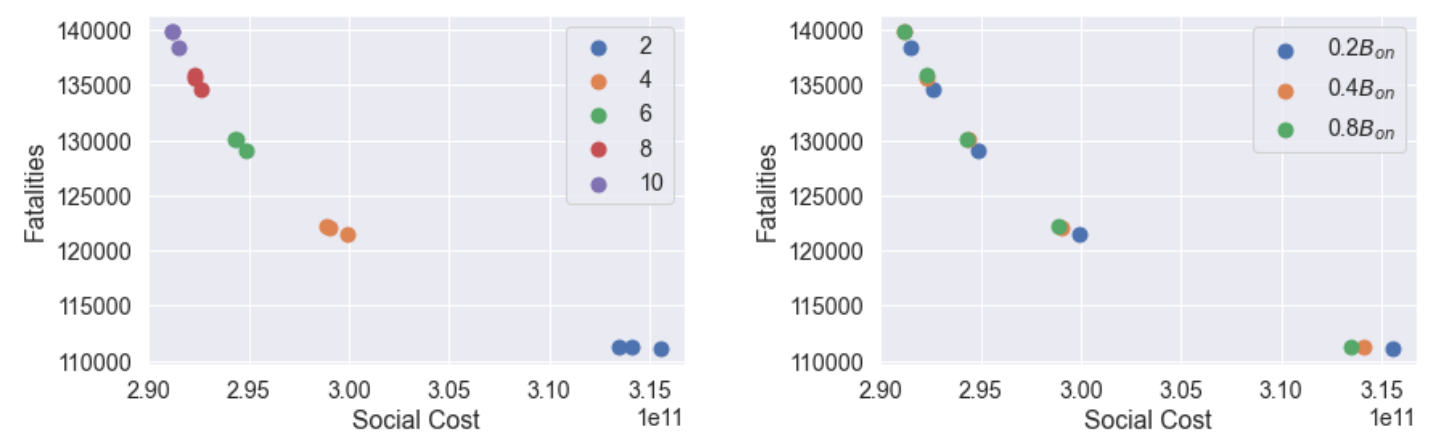

(a) Influence of the threshold $B_{\text {on }}$ for trig- (b) Influence of the threshold $B_{\text {off }}$ for lifting gering lockdown. lockdown.

Figure 23: Decentralised confinement triggered by regional daily case numbers: social cost versus fatalities $(m=0.5)$.

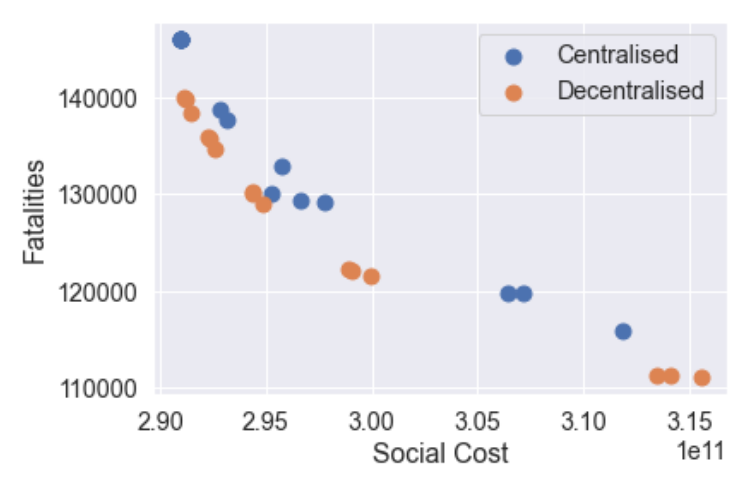

(a) Low symptomatic ratios.

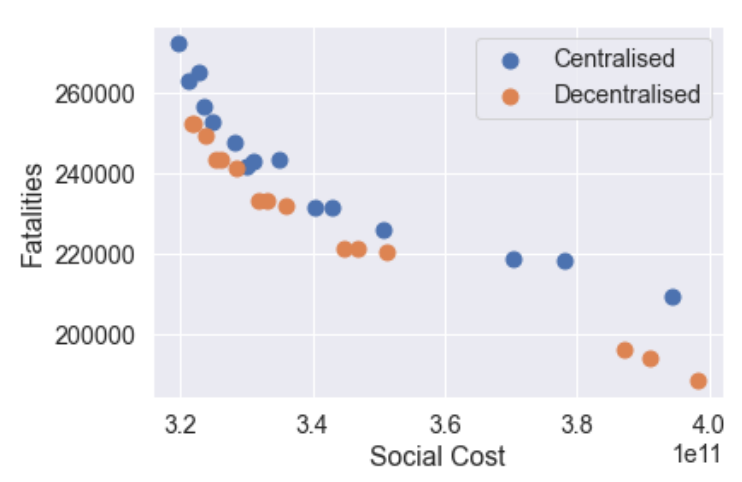

(b) High symptomatic ratios.

Figure 24: Efficiency analysis for centralised (blue) and decentralised (orange) adaptive mitigation policies. Outcomes are averaged across 100 simulated scenarios.

Figure 24 compares the outcomes of centralised and decentralised triggering policies. Decentralised policies are observed to always improve over centralised policies.

As an example, for $B_{\text {on }}=4$ and $B_{\text {off }}=0.4 B_{\text {on }}$ fatalities in England are 133, 000 under the centralised policy and 122,000 under the decentralised policy, that is $8 \%$ lower.

Figure 26 compares regional fatalities per 100,000 habitants for these policies. For more than $90 \%$ of the regions, decentralised measures lead to fewer fatalities. The most effective reductions are in Dorset, South West England (UKK22) with $23 \%$ fewer fatalities and in Cornwall and Isles of Scilly (UKK30) with $21 \%$ fewer fatalities. There are a few exceptions (see regions in light blue in Figure 26c). These regions are already under control before adaptive policies are applied. Therefore the improvement of moving from centralised policy to decentralised policy is limited.

Figure 27a compares the dynamics of symptomatic infections $\left(I_{t}\right)$ for the same example. There is a reduction of 100,000 in the amplitude of the second peak value when moving from the centralised policy to decentralised one. Decentralised policy also damps the second- 
peak values in most of the regions. Similar effects are observed for York (Figure 27c and Leicester (Figure 27b).

On June 29, 2020, Leicester became the first city in Britain to be placed in a local lockdown, after public health officials voiced concern at the city's alarming rise in COVID19 cases. Earlier in June, the Government announced that parts of the city would be released from lockdown, while a 'targeted' approach will see pockets remain under tighter restrictions. Our simulations indicate a $60 \%$ reduction of the second-peak value in Leicester when a decentralised policy is implemented (Figure 27b).

Example Figure 25 shows an example of such a decentralised triggering policy, with the same triggering thresholds as in the centralised example in Figure 19. At regional level, we see in Figure 25a that this policy is more successful than the centralised policy in taming the local outbreaks in Leicester, substantially reducing the second peak through 4 one-week regional lockdowns. At the national level this results in a strong damping of 'second wave' infections, as shown in Figure 25b (compare with Figure 19a).

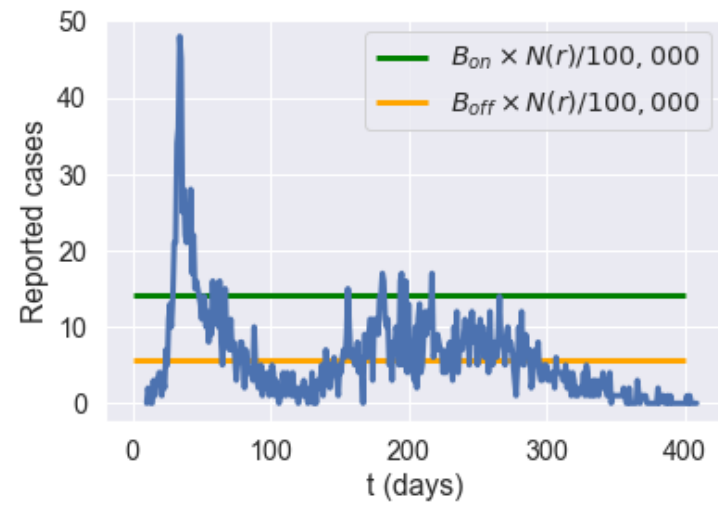

(a) Daily reported cases in Leicester.

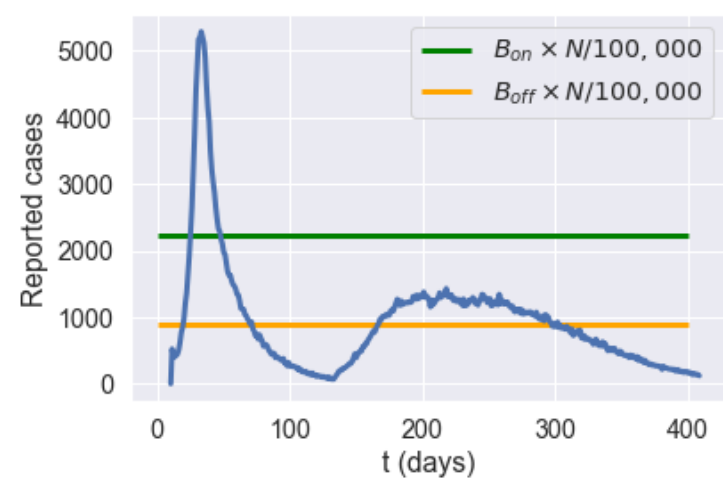

(b) Daily reported cases in England.

Figure 25: Reported cases in England and Leicester under a decentralised triggering policy: average of 50 simulated scenarios with $B_{\text {on }}=4, B_{\text {off }}=0.4 \times R_{o n}, m=0.5$, no shielding. 

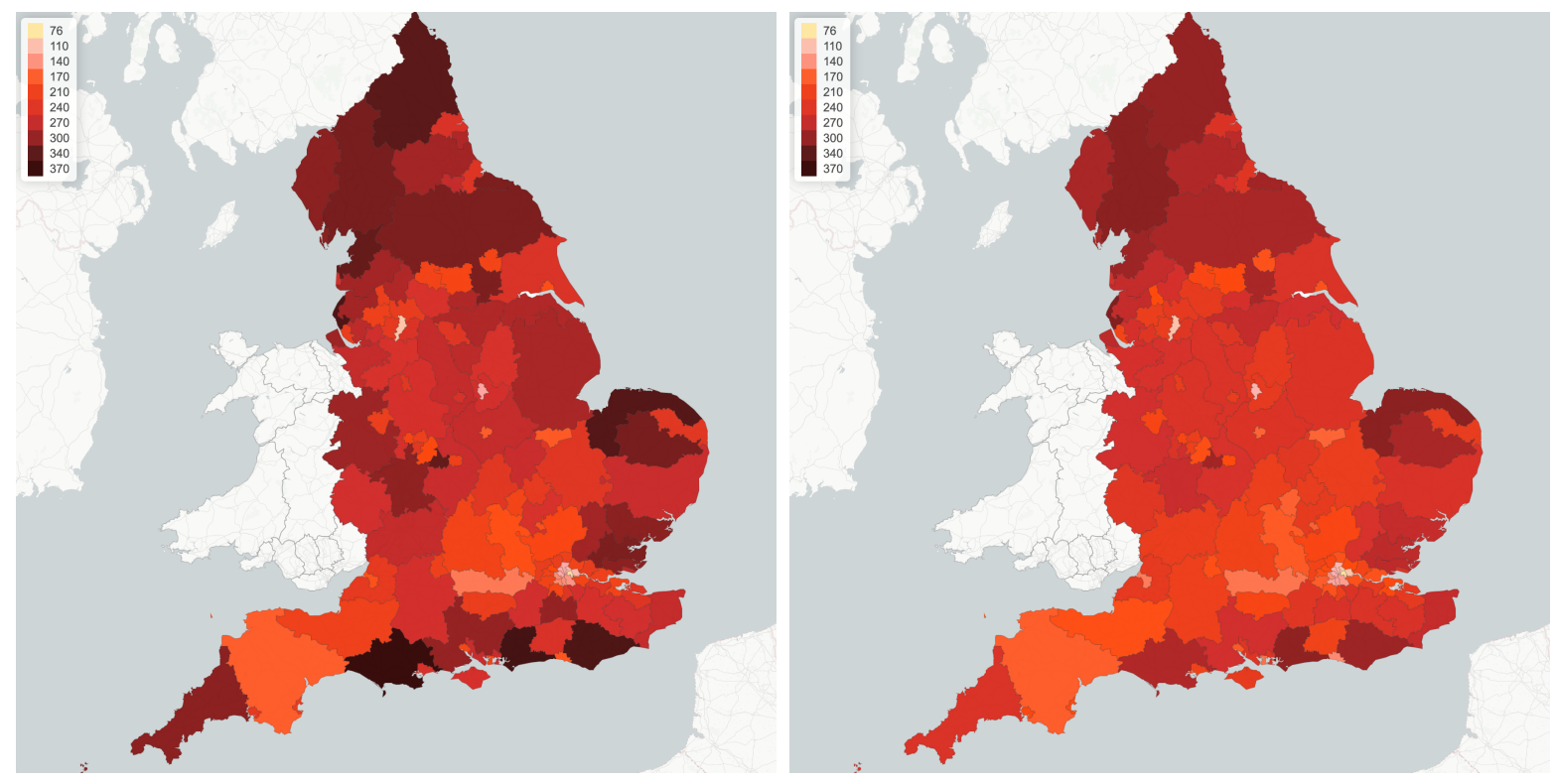

(a) Centralised (country-level) adaptive policy. (b) Decentralised (regional) adaptive policy.

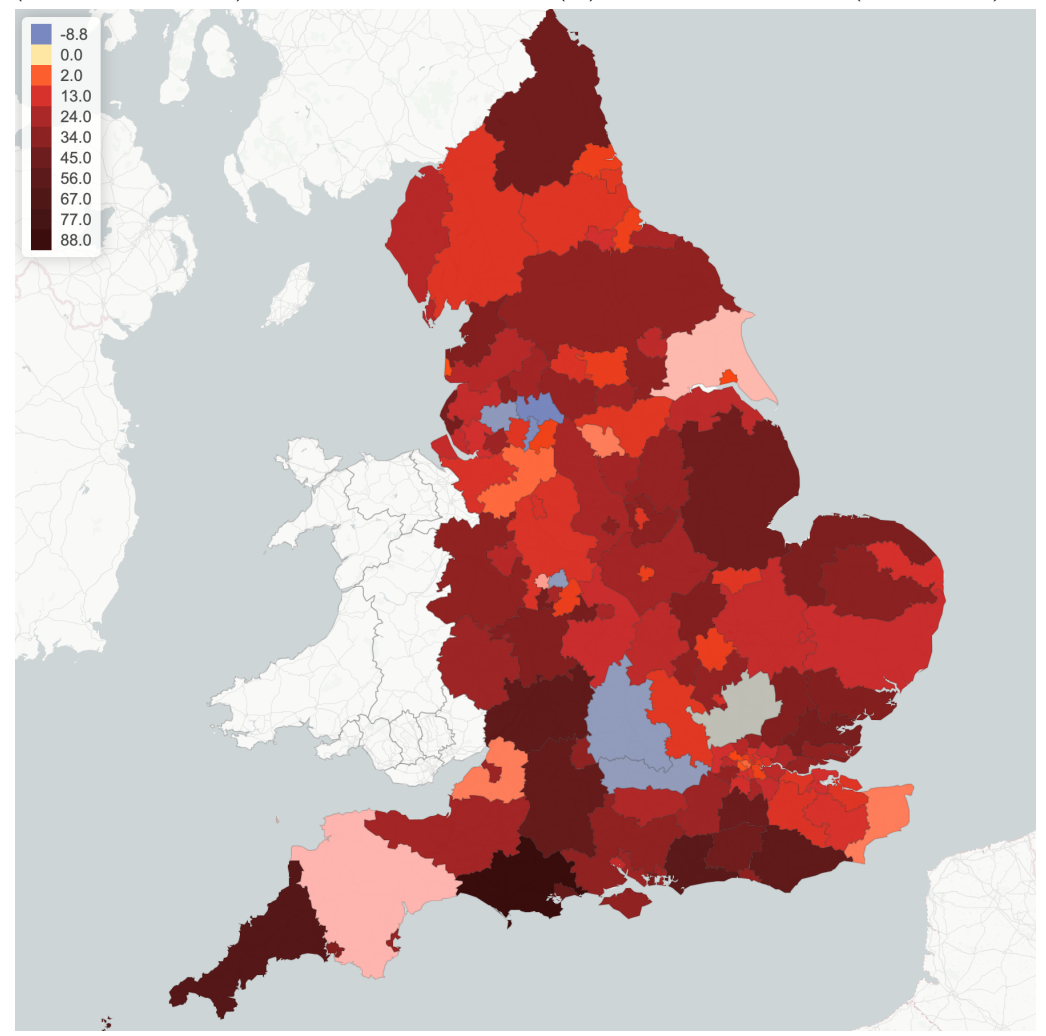

(c) Increase in fatalities (per 100,000 inhabitants) when moving from regional to centralised policy.

Figure 26: Fatalities per 100,000 inhabitants for centralised (left) verus regional (right) adaptive mitigation policies. Same triggering thresholds are used in both cases: $B_{\text {on }}=4$ and $B_{\text {off }}=0.4 B_{\text {on }}$. 


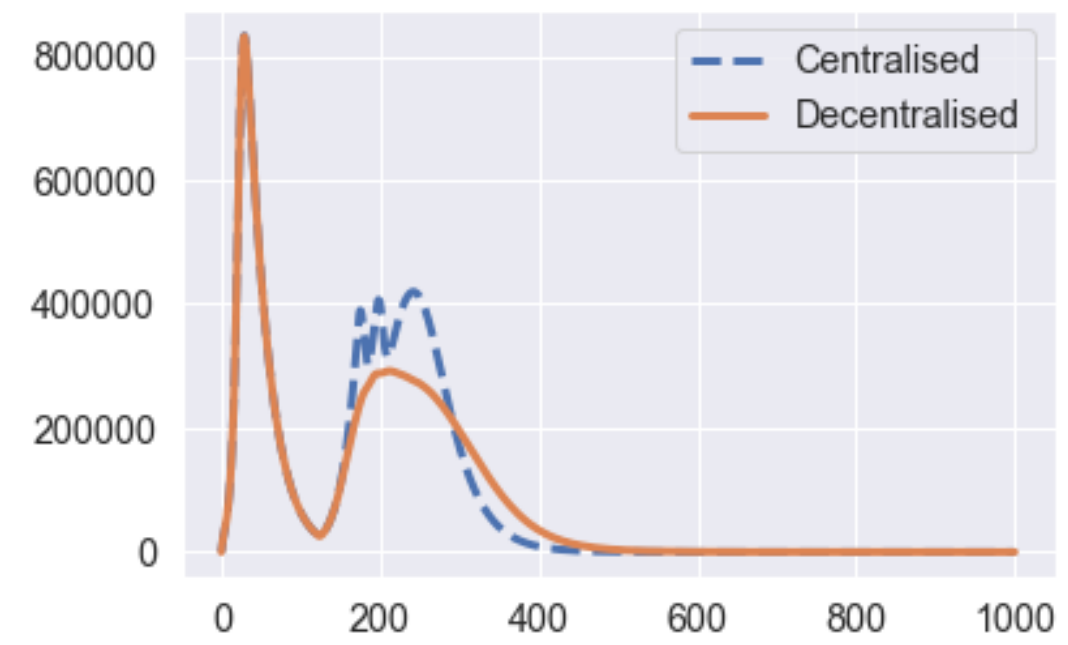

(a) Number of symptomatic individuals $\left(I_{t}\right)$ in England under centralised and decentralised policies.
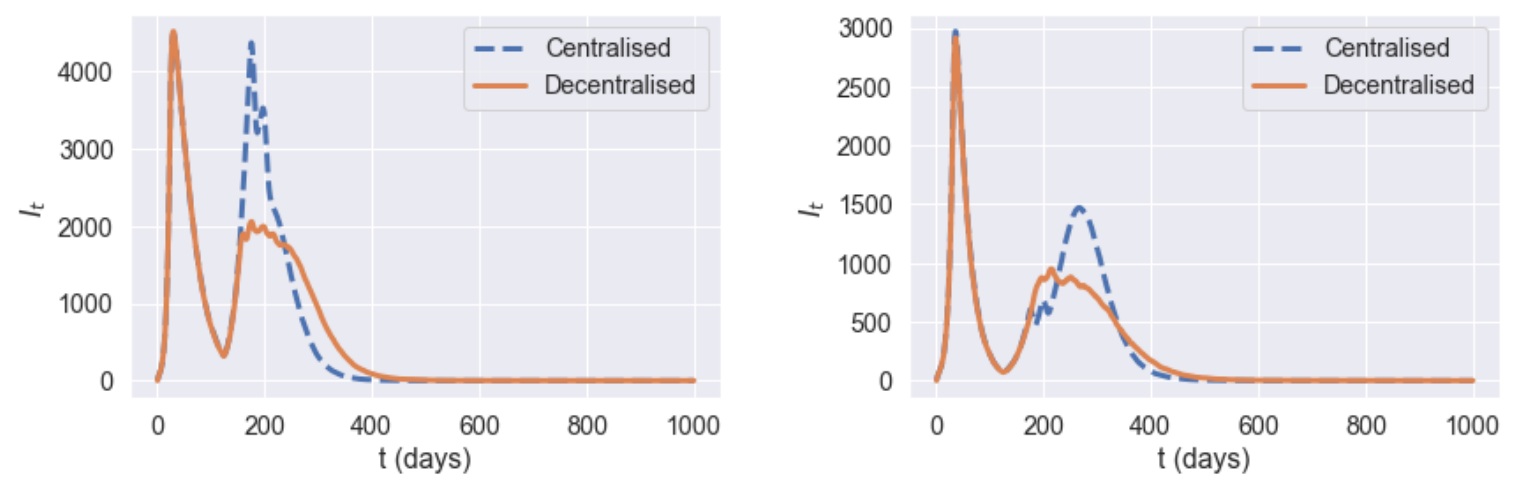

(b) Number of symptomatic individuals $\left(I_{t}\right)$ in (c) Number of symptomatic individuals $\left(I_{t}\right)$ in Leicester (UKF21). York (UKE21).

Figure 27: Number of infected individuals under under centralised (blue dashed line) and decentralised (orange solid line) policies. Same triggering thresholds are used in both cases: $B_{\text {on }}=4$ and $B_{\text {off }}=0.4 B_{\text {on }}$. 


\subsection{Adaptive versus pre-planned policies}

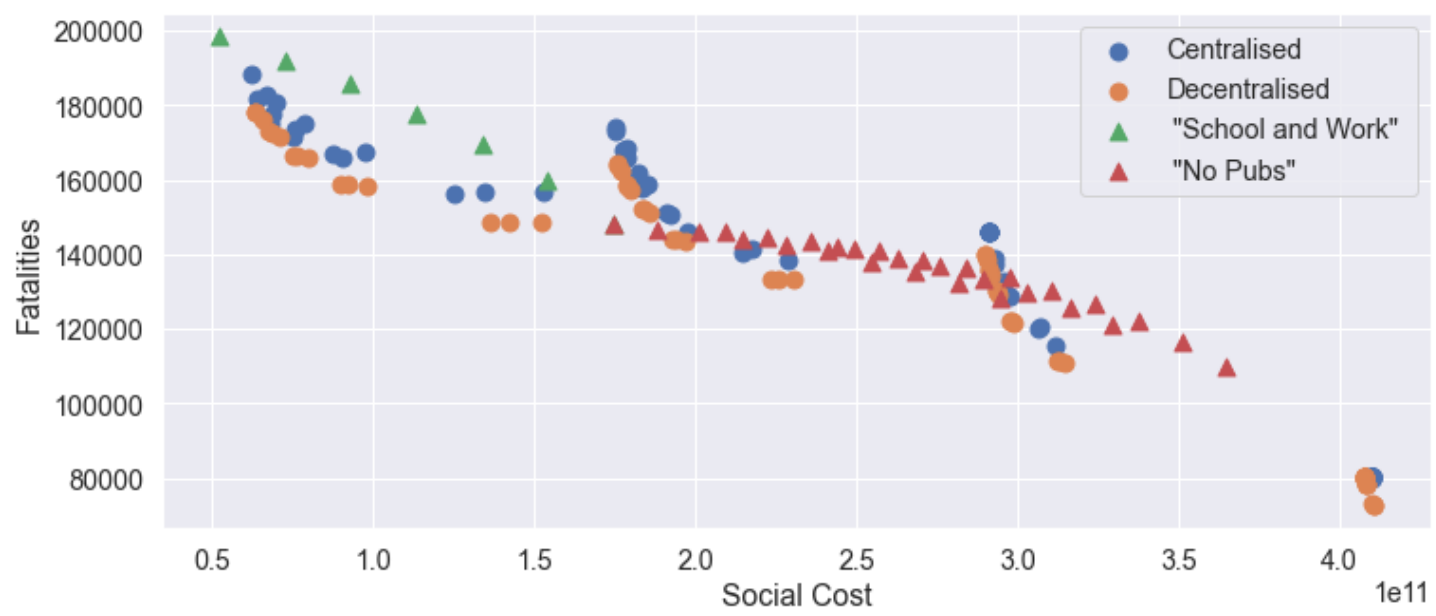

(a) No Shielding.

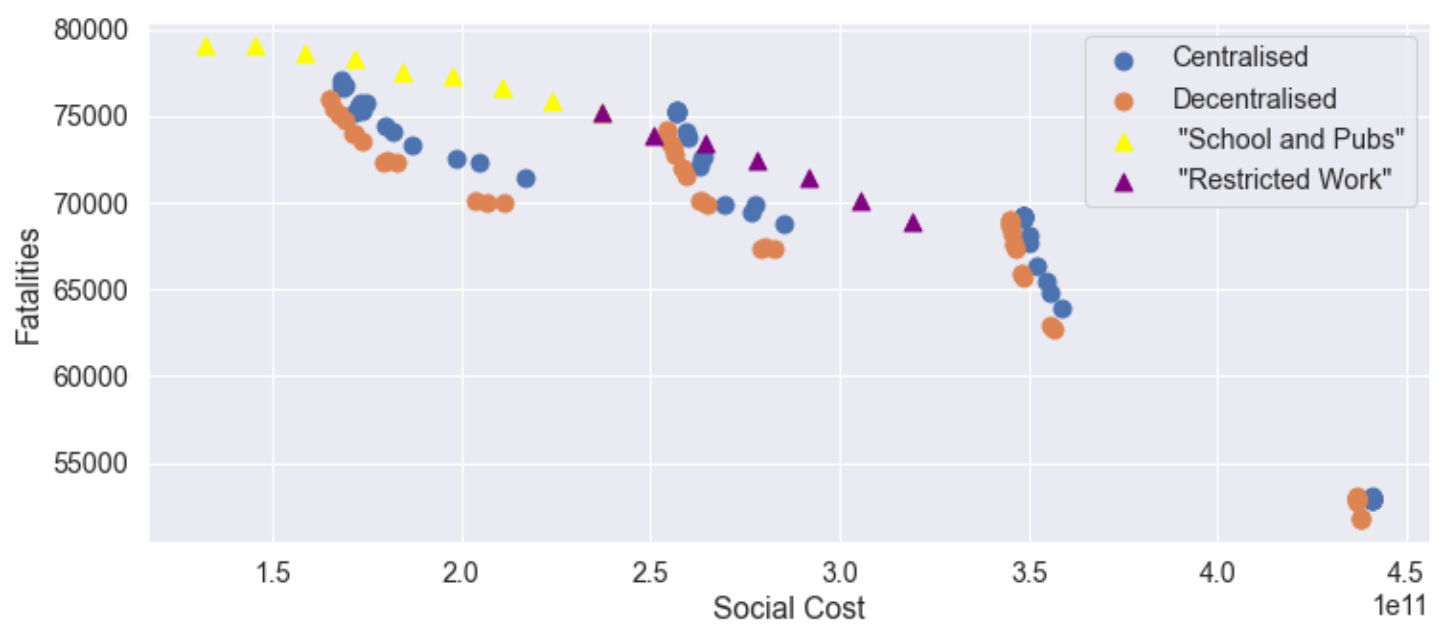

(b) Shielding.

Figure 28: Efficiency plot: pre-planned versus adaptive mitigation policies.

Figure 28 compares the health outcome and social cost of the efficient policies considered in Sections 5.2, 6.1 and 6.2. The efficient frontier of pre-planned policies are among policies with $u^{S} \in\{0,0.5,1\}, 0.2 \leq u^{W} \leq 1.0$ and $0.3 \leq u^{O} \leq 1.0$. For centralised and decentralised policies, $m=0.25,0.5,0.75,1 ; B_{\text {on }}=2,4,6,8,10$; and $B_{\text {off }}=p \times B_{\text {on }}$ with $p=0.2,0.4,0.8$.

We observe that

- adaptive policies, in which measures are triggered when the number of daily new cases exceeds a threshold, are more efficient than pre-planned policies; and

- as shown in Figures 28a and 28b, a decentralised policy is more efficient than both centralised policy and pre-planned policy. 
In Table 10, we provide a summary of outcomes for five different types of policies.

- Confinement of $T=105$ days followed by social distancing $(m=0.3$ or $m=0.5)$, no shielding.

- Pre-planned policy: social distancing at work and school $\left(u^{H}=1, u^{S}=0.5\right.$, $\left.u^{W}=0.5\right)$, restrictions on social gatherings $\left(u^{O}=0.3\right)$ and no shielding.

- Centralised and decentralised triggering policies (Section 6.1 and 6.2 with $m=0.5$, $B_{\text {on }}=4, B_{\text {off }}=0.4 B_{\text {on }}$ and no shielding;

- Decentralised triggering combined with shielding of elderly populations: $m=0.5$, $B_{\text {on }}=4, B_{\text {off }}=0.4 B_{\text {on }}$;

- 'Protect Lives' policy: in the range of efficient policies, the one which results in the fewest fatalities is a decentralised triggering policy with $B_{\text {on }}=2, B_{\text {off }}=0.2 B_{\text {on }}$ (so more frequent triggering of confinement measures than the above), high degree of social distancing $(m=0.25)$ and shielding of elderly populations. This policy corresponds to the point in the lower right corner of Figure 28b. The social cost is 4.52 , which is much higher than for the other considered policies.

\begin{tabular}{|l|l|l|l|l|l|l|}
\hline Policy & $I_{t}$ & $A_{t}$ & Fatalities & $\begin{array}{l}\text { max } I_{t} \\
\left(2^{\text {nd }} \text { peak }\right)\end{array}$ & $\begin{array}{l}\text { Social } \\
\text { cost } \\
\left(10^{11}\right)\end{array}$ & $\begin{array}{l}\text { Projected } \\
\text { fatalities } \\
(1000 \text { days })\end{array}$ \\
\hline $\begin{array}{l}\text { Confinement followed } \\
\text { by strict social dis- } \\
\text { tancing (m=0.3) }\end{array}$ & 47,400 & 188,700 & 39,400 & 255,700 & 3.8 & 96,600 \\
\hline $\begin{array}{l}\text { Confinement followed } \\
\text { by moderate social } \\
\text { distancing (m=0.5) }\end{array}$ & 98,400 & 392,400 & 40,700 & 766,800 & 2.9 & 146,100 \\
\hline Pre-planned & 84,700 & 360,100 & 45,500 & 613,300 & 2.9 & 122,900 \\
\hline Centralised triggering & 80,300 & 321,200 & 40,500 & 423,200 & 3.0 & 133,500 \\
\hline $\begin{array}{l}\text { Decentralised trigger- } \\
\text { ing }\end{array}$ & 80,100 & 320,200 & 40,400 & 292,200 & 3.0 & 122,100 \\
\hline $\begin{array}{l}\text { Decentralised trigger- } \\
\text { ing and shielding }\end{array}$ & 55,000 & 266,100 & 39,700 & 267,700 & 3.4 & 65,900 \\
\hline 'Protect Lives' & 25,900 & 118,400 & 39,600 & 63,900 & 4.3 & 51,700 \\
\hline
\end{tabular}

Table 10: Summary of outcomes for different policies, starting from the same initial conditions on July 4, 2020.

Outcomes are averaged across 50 scenarios, starting from the same initial conditions on July 4 (end of the UK lockdown). 
Regional outcomes Comparing the regional outcomes of the centralised, decentralised and pre-planned policies displayed in Table 10 shows that the decentralised triggering policies are able in many cases to considerably damp the 'second wave' of infections. Figure 29 illustrates this in the case of Mid Lancashire, York, Leicester and Birmingham: the decentralised triggering policy reduces the second peak amplitude by around one half compared to the pre-planned policy.

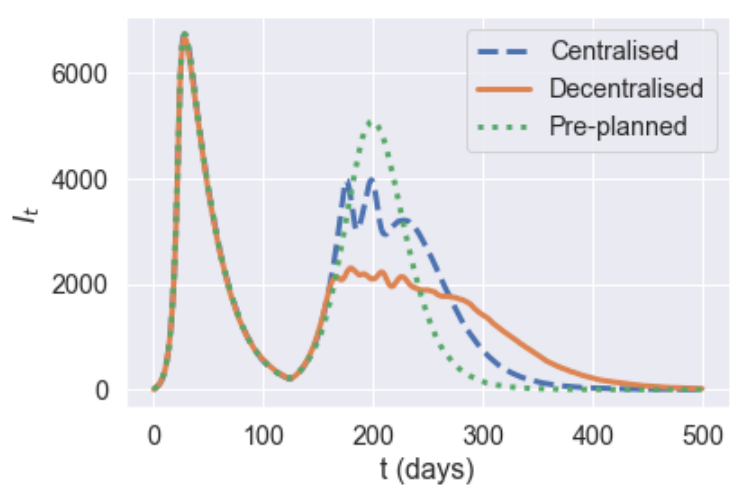

(a) $I_{t}$ in Mid Lancashire.

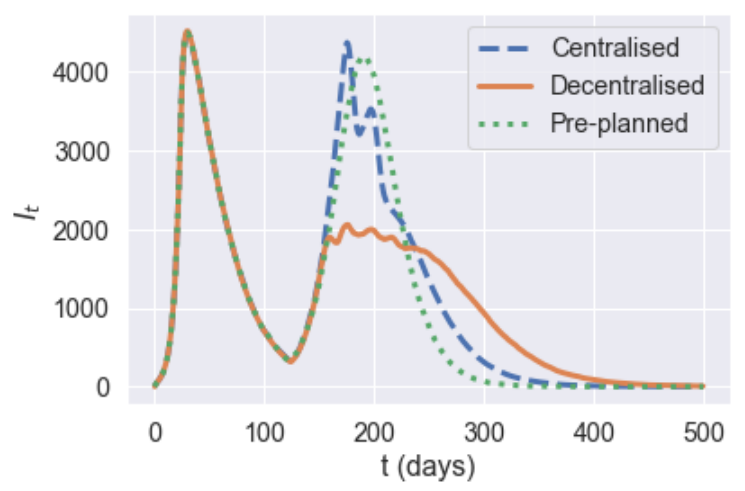

(c) $I_{t}$ in Leicester.

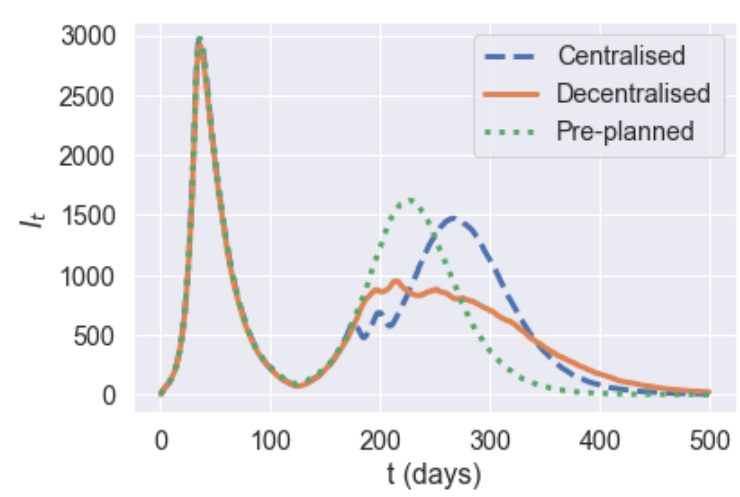

(b) $I_{t}$ in York.

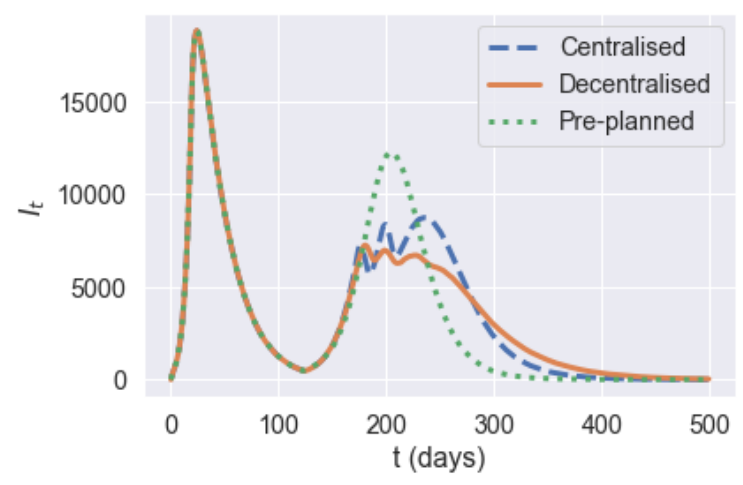

(d) $I_{t}$ in Birmingham.

Figure 29: Regional comparison of pre-planned and adaptive mitigation policies.

\section{References}

[1] Daron Acemoglu, Victor Chernozhukov, Iván Werning, and Michael D Whinston. A multi-risk SIR model with optimally targeted lockdown. Technical report, National Bureau of Economic Research, 2020.

[2] R Adhikari, Austen Bolitho, Fernando Caballero, Michael E Cates, Jakub Dolezal, Timothy Ekeh, Jules Guioth, Robert L Jack, Julian Kappler, Lukas Kikuchi, et al. Inference, prediction and optimization of non-pharmaceutical interventions using compartment models: the PyRoss library. arXiv preprint arXiv:2005.09625, 2020.

[3] Jacob Aguilar, Jeremy Samuel Faust, Jeremy Samuel Faust, Lauren Westafer, and 
Juan Gutierrez. A model describing COVID-19 community transmission taking into account asymptomatic carriers and risk mitigation. medRxiv, 2020.

[4] Linda J.S. Allen. A primer on stochastic epidemic models: Formulation, numerical simulation, and analysis. Infectious Disease Modelling, 2(2):128 - 142, 2017.

[5] R.M. Anderson and R.M. May. Infectious Diseases of Humans: Dynamics and Control. Oxford University Press, 1991.

[6] Jantien A Backer, Don Klinkenberg, and Jacco Wallinga. Incubation period of 2019 novel coronavirus (2019-ncov) infections among travellers from Wuhan, China. Eurosurveillance, 25(5), 2020.

[7] Linlin Bao, Wei Deng, Hong Gao, Chong Xiao, Jiayi Liu, Jing Xue, Qi Lv, Jiangning Liu, Pin Yu, Yanfeng Xu, Feifei Qi, Yajin Qu, Fengdi Li, Zhiguang Xiang, Haisheng Yu, Shuran Gong, Mingya Liu, Guanpeng Wang, Shunyi Wang, Zhiqi Song, Wenjie Zhao, Yunlin Han, Linna Zhao, Xing Liu, Qiang Wei, and Chuan Qin. Reinfection could not occur in SARS-CoV-2 infected rhesus macaques. bioRxiv, 2020.

[8] Guillaume Béraud, Sabine Kazmercziak, Philippe Beutels, Daniel Levy-Bruhl, Xavier Lenne, Nathalie Mielcarek, Yazdan Yazdanpanah, Pierre-Yves Boëlle, Niel Hens, and Benoit Dervaux. The French connection: the first large population-based contact survey in france relevant for the spread of infectious diseases. PLoS one, 10(7), 2015.

[9] John R Birge, Ozan Candogan, and Yiding Feng. Controlling epidemic spread: Reducing economic losses with targeted closures. Working Paper 2020-57, University of Chicago, 2020.

[10] Fred Brauer and Carlos Castillo-Chavez. Mathematical Models for Communicable Diseases. Society for Industrial and Applied Mathematics, Philadelphia, PA, 2012.

[11] Tom Britton, Etienne Pardoux, Franck Ball, Catherine Laredo, David Sirl, and Viet Chí Tran. Stochastic epidemic models with inference. Springer, 2019.

[12] Diana Buitrago-Garcia, Michel Counotte, Dianne Egli-Gany, Stefanie Hossmann, Hira Imeri, and Nicola Low. The role of asymptomatic SARS-CoV-2 infections: A rapid systematic review, April 2020.

[13] Zhidong Cao, Qingpeng Zhang, Xin Lu, Dirk Pfeiffer, Zhongwei Jia, Hongbing Song, and Daniel Dajun Zeng. Estimating the effective reproduction number of the 2019ncov in china. medRxiv, 2020.

[14] Maria Chikina and Wesley Pegden. Modeling strict age-targeted mitigation strategies for covid-19. PLoS ONE, 15(7), 2020.

[15] Leon Danon, Ellen Brooks-Pollock, Mick Bailey, and Matt J Keeling. A spatial model of covid-19 transmission in England and Wales: early spread and peak timing. Technical report, 2020. 
[16] Nicholas G Davies, Petra Klepac, Yang Liu, Kiesha Prem, Mark Jit, Rosalind M Eggo, and CMMID COVID-19 working group. Age-dependent effects in the transmission and control of COVID-19 epidemics. Nat Med, 2020.

[17] Claire Donnat and Susan Holmes. Modeling the heterogeneity in COVID-19 reproductive number and its impact on predictive scenarios. arXiv:2004.05272, 2020.

[18] Ilaria Dorigatti, Lucy Okell, Anne Cori, Natsuko Imai, Marc Baguelin, Sangeeta Bhatia, Adhiratha Boonyasiri, Z Cucunubá, G Cuomo-Dannenburg, R FitzJohn, et al. Severity of 2019-novel coronavirus (ncov). Technical report, Imperial College London, 2020 .

[19] Eurostat. Population on 1 January by age group, sex and NUTS 3 region [computer file]. European Commission. Downloaded from: https://appsso.eurostat.ec. europa.eu/nui/show.do?dataset=demo_r_pjangrp3\&lang=en, January 2020. Table demo_r_pjangrp3.

[20] Eurostat. NUTS - Nomenclature Of Territorial Units For Statistics. European Commission. Available at: https://ec.europa.eu/eurostat/web/nuts/background, January 2020.

[21] C Farquharson, I Rasul, and L Sibieta. Key workers: key facts and questions. IFS Observation, Institute for Fiscal Studies, 20, 2020.

[22] Neil Ferguson, Daniel Laydon, Gemma Nedjati Gilani, Natsuko Imai, Kylie Ainslie, Marc Baguelin, Sangeeta Bhatia, Adhiratha Boonyasiri, Zulma Cucunuba-Perez, Gina Cuomo-Dannenburg, et al. Report 9: Impact of non-pharmaceutical interventions (npis) to reduce COVID-19 mortality and healthcare demand. 2020.

[23] Seth Flaxman, Swapnil Mishra, Axel Gandy, et al. Estimating the number of infections and the impact of non-pharmaceutical interventions on COVID-19 in 11 European countries. Technical Report 30, Imperial College COVID-19 Response Team, 2020.

[24] Christian Gourieroux, Alain Monfort, and Eric Renault. Indirect inference. Journal of applied econometrics, 8(S1):S85-S118, 1993.

[25] Valerie Isham. Assessing the variability of stochastic epidemics. Mathematical biosciences, 107(2):209-224, 1991.

[26] Thibaut Jombart, Kevin Van Zandvoort, Timothy W Russell, Christopher I Jarvis, Amy Gimma, Sam Abbott, Sam Clifford, Sebastian Funk, Hamish Gibbs, Yang Liu, et al. Inferring the number of covid-19 cases from recently reported deaths. Wellcome Open Research, 5, 2020.

[27] Malahat Khalili, Mohammad Karamouzian, Naser Nasiri, Sara Javadi, Ali Mirzazadeh, and Hamid Sharifi. Epidemiological characteristics of COVID-19: A systemic review and meta-analysis. medRxiv, 2020. 
[28] Petra Klepac, Adam J Kucharski, Andrew JK Conlan, Stephen Kissler, Maria Tang, Hannah Fry, and Julia R Gog. Contacts in context: large-scale setting-specific social mixing matrices from the bbc pandemic project. medRxiv, 2020.

[29] Adam J Kucharski, Timothy W Russell, Charlie Diamond, Yang Liu, John Edmunds, Sebastian Funk, Rosalind M Eggo, Fiona Sun, Mark Jit, James D Munday, Nicholas Davies, Amy Gimma, Kevin van Zandvoort, Hamish Gibbs, Joel Hellewell, Christopher I Jarvis, Sam Clifford, Billy J Quilty, Nikos I Bosse, Sam Abbott, Petra Klepac, and Stefan Flasche. Early dynamics of transmission and control of COVID-19: a mathematical modelling study. The Lancet Infectious Diseases, 20(5):553-558, 2020.

[30] Stephen A Lauer, Kyra H Grantz, Qifang Bi, Forrest K Jones, Qulu Zheng, Hannah R Meredith, Andrew S Azman, Nicholas G Reich, and Justin Lessler. The incubation period of coronavirus disease 2019 (COVID-19) from publicly reported confirmed cases: estimation and application. Annals of internal medicine, 172(9):577-582, 2020.

[31] Qun Li, Xuhua Guan, Peng Wu, Xiaoye Wang, Lei Zhou, Yeqing Tong, Ruiqi Ren, Kathy SM Leung, Eric HY Lau, Jessica Y Wong, et al. Early transmission dynamics in Wuhan, China, of novel coronavirus-infected pneumonia. New England Journal of Medicine, 382:1199-1207, 2020.

[32] Natalie M Linton, Tetsuro Kobayashi, Yichi Yang, Katsuma Hayashi, Andrei R Akhmetzhanov, Sung-mok Jung, Baoyin Yuan, Ryo Kinoshita, and Hiroshi Nishiura. Incubation period and other epidemiological characteristics of 2019 novel coronavirus infections with right truncation: a statistical analysis of publicly available case data. Journal of clinical medicine, 9(2):538, 2020.

[33] Marc Lipsitch, Christl A. Donnelly, Christophe Fraser, Isobel M. Blake, Anne Cori, Ilaria Dorigatti, Neil M. Ferguson, Tini Garske, Harriet L. Mills, Steven Riley, Maria D. Van Kerkhove, and Miguel A. Hernan. Potential biases in estimating absolute and relative case-fatality risks during outbreaks. PLOS Neglected Tropical Diseases, 9 (7):1-16, July 2015. doi: 10.1371/journal.pntd.0003846.

[34] Alex Lipton and Marcos Lopez de Prado. Mitigation strategies for COVID-19: Lessons from the K-SEIR model. Working paper, 2020.

[35] Alun L Lloyd and Vincent A.A Jansen. Spatiotemporal dynamics of epidemics: synchrony in metapopulation models. Mathematical Biosciences, 188(1):1-16, 2004.

[36] Jose Lourenco, Robert Paton, Mahan Ghafari, Moritz Kraemer, Craig Thompson, Peter Simmonds, Paul Klenerman, and Sunetra Gupta. Fundamental principles of epidemic spread highlight the immediate need for large-scale serological surveys to assess the stage of the SARS-CoV-2 epidemic. MedRxiv, 2020.

[37] Kenji Mizumoto, Katsushi Kagaya, Alexander Zarebski, and Gerardo Chowell. Estimating the asymptomatic proportion of Coronavirus disease 2019 (COVID-19) cases on board the Diamond Princess cruise ship, Yokohama, Japan, 2020. Eurosurveillance, 25(10):2000180, 2020. 
[38] Joel Mossong, Niel Hens, Mark Jit, Philippe Beutels, Kari Auranen, Rafael Mikolajczyk, Marco Massari, Stefania Salmaso, Gianpaolo Scalia Tomba, Jacco Wallinga, Janneke Heijne, Malgorzata Sadkowska-Todys, Magdalena Rosinska, and W. John Edmunds. Social contacts and mixing patterns relevant to the spread of infectious diseases. PLOS Medicine, 5(3):1-1, March 2008.

[39] Joel Mossong, Niel Hens, Mark Jit, Philippe Beutels, Kari Auranen, Rafael Mikolajczyk, Marco Massari, Stefania Salmaso, Gianpaolo Scalia Tomba, Jacco Wallinga, Janneke Heijne, Malgorzata Sadkowska-Todys, Magdalena Rosinska, and W. John Edmunds. Polymod social contact data, 2017. Version 1.1.

[40] Hiroshi Nishiura, Tetsuro Kobayashi, Ayako Suzuki, Sung-Mok Jung, Katsuma Hayashi, Ryo Kinoshita, Yichi Yang, Baoyin Yuan, Andrei R. Akhmetzhanov, Natalie M. Linton, and Takeshi Miyama. Estimation of the asymptomatic ratio of novel coronavirus infections (covid-19). International Journal of Infectious Diseases, 2020.

[41] Office for National Statistics. Coronavirus (COVID-19) infections in the community in England: July 2020. Downloaded from: https://www.ons.gov.uk/peoplepopulationandcommunity/ healthandsocialcare/conditionsanddiseases/articles/ coronaviruscovid19infectionsinthecommunityinengland/july2020, January 2020 .

[42] Office for National Statistics. 2011 Census: Special Workplace Statistics (United Kingdom) [computer file]. UK Data Service Census Support. Downloaded from: https://wicid.ukdataservice.ac.uk, January 2020. Table WU01EW.

[43] Office for National Statistics. LAU2 to LAU1 to NUTS3 to NUTS2 to NUTS1 (January 2018) Lookup in United Kingdom v3 [computer file]. Downloaded from: https://geoportal.statistics.gov.uk/datasets/ c893dfece45f465f857ac34641041863_0, January 2020.

[44] Robert S Pindyck. Covid-19 and the welfare effects of reducing contagion. Technical report, National Bureau of Economic Research, 2020.

[45] Kiesha Prem, Alex R Cook, and Mark Jit. Projecting social contact matrices in 152 countries using contact surveys and demographic data. PLoS computational biology, 13(9):e1005697, 2017.

[46] Kiesha Prem, Yang Liu, Timothy W Russell, Adam J Kucharski, Rosalind M Eggo, Nicholas Davies, Stefan Flasche, Samuel Clifford, Carl AB Pearson, James D Munday, et al. The effect of control strategies to reduce social mixing on outcomes of the COVID-19 epidemic in wuhan, china: a modelling study. The Lancet Public Health, 5(5):261-270, May 2020.

[47] Public Health England and NHSX. GOV.UK Coronavirus (COVID-19) in the UK. Downloaded from: https://coronavirus.data.gov.uk, June 2020. 
[48] Thomas Rawson, Tom Brewer, Dessislava Veltcheva, Chris Huntingford, and Michael B. Bonsall. How and when to end the covid-19 lockdown: An optimization approach. Frontiers in Public Health, 8:262, 2020.

[49] Joacim Rocklöv, Henrik Sjödin, and Annelies Wilder-Smith. COVID-19 outbreak on the diamond princess cruise ship: estimating the epidemic potential and effectiveness of public health countermeasures. Journal of travel medicine, 2020.

[50] Lionel Roques, Olivier Bonnefon, Virgile Baudrot, Samuel Soubeyrand, and Henri Berestycki. A parsimonious model for spatial transmission and heterogeneity in the covid-19 propagation. medRxiv, 2020.

[51] Lionel Roques, Etienne K. Klein, Julien Papaix, Antoine Sar, and Samuel Soubeyrand. Impact of lockdown on the epidemic dynamics of COVID-19 in France. Frontiers in Medicine, 7:274, 2020.

[52] Robert Rowthorn and Jan Maciejowski. A cost-benefit analysis of the Covid-19 disease. Oxford Review of Economic Policy, 2020.

[53] Henrik Salje, Cécile Tran Kiem, Noémie Lefrancq, Noémie Courtejoie, Paolo Bosetti, Juliette Paireau, Alessio Andronico, Nathanaël Hozé, Jehanne Richet, Claire-Lise Dubost, Yann Le Strat, Justin Lessler, Daniel Levy-Bruhl, Arnaud Fontanet, Lulla Opatowski, Pierre-Yves Boelle, and Simon Cauchemez. Estimating the burden of SARSCoV-2 in France. Science, 369(6500):208-211, 2020.

[54] Clodomir Santana, Federico Botta, Hugo Barbosa, Filippo Privitera, Ronaldo Menezes, and Riccardo Di Clemente. Analysis of human mobility in the UK during the COVID-19 pandemic. 2020.

[55] Rajesh Singh and Ronojoy Adhikari. Age-structured impact of social distancing on the COVID-19 epidemic in india. arxiv:2003.12055, 2020.

[56] Robert Verity, Lucy C Okell, Ilaria Dorigatti, Peter Winskill, Charles Whittaker, Natsuko Imai, Gina Cuomo-Dannenburg, Hayley Thompson, Patrick GT Walker, Han $\mathrm{Fu}$, et al. Estimates of the severity of coronavirus disease 2019: a model-based analysis. The Lancet Infectious Diseases, 20(6):669-677, 2020. 


\section{A Demographic regions}

Table 11 details the used mapping between Upper Tier Local Authority (UTLA) region codes and the Nomenclature of Territorial Units for Statistics at level 3 (NUTS-3) codes 7 . If more than one UTLA region falls within the boundary of a single NUTS-3 region, the data is then aggregated. On the other hand, if a single UTLA region lies within more than one NUTS-3 region, the data is distributed among NUTS-3 regions in proportion to the total number of people living in each region.

\begin{tabular}{|l|l|l|}
\hline UTLA Code & UTLA Region Name & NUTS-3 Code Mapping \\
\hline \hline E06000001 & Hartlepool & UKC11 \\
\hline E0600002 & Middlesbrough & UKC12 \\
\hline E06000003 & Redcar and Cleveland & UKC12 \\
\hline E0600004 & Stockton-on-Tees & UKC11 \\
\hline E06000005 & Darlington & UKC13 \\
\hline E0600006 & Halton & UKD71 \\
\hline E06000007 & Warrington & UKD61 \\
\hline E06000008 & Blackburn with Darwen & UKD41 \\
\hline E06000009 & Blackpool & UKD42 \\
\hline E06000010 & Kingston upon Hull, City of & UKE11 \\
\hline E06000011 & East Riding of Yorkshire & UKE12 \\
\hline E06000012 & North East Lincolnshire & UKE13 \\
\hline E06000013 & North Lincolnshire & UKE13 \\
\hline E06000014 & York & UKE21 \\
\hline E06000015 & Derby & UKF11 \\
\hline E06000016 & Leicester & UKF21 \\
\hline E06000017 & Rutland & UKF22 \\
\hline E06000018 & Nottingham & UKF14 \\
\hline E06000019 & Herefordshire, County of & UKG11 \\
\hline E06000020 & Telford and Wrekin & UKG21 \\
\hline E06000021 & Stoke-on-Trent & UKG23 \\
\hline E06000022 & Bath and North East Somerset & UKK12 \\
\hline E06000023 & Bristol, City of & UKK11 \\
\hline E06000024 & North Somerset & UKK12 \\
\hline E06000025 & South Gloucestershire & UKK12 \\
\hline E06000026 & Plymouth & UKK41 \\
\hline E06000027 & Torbay & UKK42 \\
\hline E06000030 & Swindon & UKK14 \\
\hline E06000031 & Peterborough & UKH11 \\
\hline E06000032 & Luton & UKH21 \\
\hline E06000033 & Southend-on-Sea & UKH31 \\
\hline E06000034 & Thurrock & UKH32 \\
\hline E06000035 & Medway & UKJ41 \\
\hline E06000036 & Bracknell Forest & UKJ11 \\
\hline E06000037 & West Berkshire & UKJ11 \\
\hline E06000038 & Reading & UKJ11 \\
\hline E06000039 & Slough & UKJ11 \\
\hline E06000040 & Windsor and Maidenhead & UKJ11 \\
\hline
\end{tabular}

\footnotetext{
${ }^{7}$ See https://geoportal.statistics.gov.uk/datasets/c893dfece45f465f857ac34641041863_0 for a lookup table used in the process of mapping.
} 


\begin{tabular}{|c|c|c|}
\hline E06000041 & Wokingham & UKJ11 \\
\hline E06000042 & Milton Keynes & UKJ12 \\
\hline E06000043 & Brighton and Hove & UKJ21 \\
\hline E06000044 & Portsmouth & UKJ31 \\
\hline E06000045 & Southampton & UKJ32 \\
\hline E06000046 & Isle of Wight & UKJ34 \\
\hline E06000047 & County Durham & UKC14 \\
\hline E06000049 & Cheshire East & UKD62 \\
\hline E06000050 & Cheshire West and Chester & UKD63 \\
\hline E06000051 & Shropshire & UKG22 \\
\hline E06000052 & Cornwall and Isles of Scilly & UKK30 \\
\hline E06000054 & Wiltshire & UKK15 \\
\hline E06000055 & Bedford & UKH24 \\
\hline E06000056 & Central Bedfordshire & UKH25 \\
\hline E06000057 & Northumberland & UKC21 \\
\hline E06000058 & Bournemouth and Poole & UKK21 \\
\hline E06000059 & Dorset & UKK22 \\
\hline E08000001 & Bolton & UKD36 \\
\hline E08000002 & Bury & UKD37 \\
\hline E08000003 & Manchester & UKD33 \\
\hline E08000004 & Oldham & UKD37 \\
\hline E08000005 & Rochdale & UKD37 \\
\hline E08000006 & Salford & UKD34 \\
\hline E08000007 & Stockport & UKD35 \\
\hline E08000008 & Tameside & UKD35 \\
\hline E08000009 & Trafford & UKD34 \\
\hline E08000010 & Wigan & UKD36 \\
\hline E08000011 & Knowsley & UKD71 \\
\hline E08000012 & Liverpool & UKD72 \\
\hline E08000013 & St. Helens & UKD71 \\
\hline E08000014 & Sefton & UKD73 \\
\hline E08000015 & Wirral & UKD74 \\
\hline E08000016 & Barnsley & UKE31 \\
\hline E08000017 & Doncaster & UKE31 \\
\hline E08000018 & Rotherham & UKE31 \\
\hline E08000019 & Sheffield & UKE32 \\
\hline E08000021 & Newcastle upon Tyne & UKC22 \\
\hline E08000022 & North Tyneside & UKC22 \\
\hline E08000023 & South Tyneside & UKC22 \\
\hline E08000024 & Sunderland & UKC23 \\
\hline E08000025 & Birmingham & UKG31 \\
\hline E08000026 & Coventry & UKG33 \\
\hline E08000027 & Dudley & UKG36 \\
\hline E08000028 & Sandwell & UKG37 \\
\hline E08000029 & Solihull & UKG32 \\
\hline E08000030 & Walsall & UKG38 \\
\hline E08000031 & Wolverhampton & UKG39 \\
\hline E08000032 & Bradford & UKE41 \\
\hline E08000033 & Calderdale & UKE44 \\
\hline E08000034 & Kirklees & UKE44 \\
\hline
\end{tabular}




\begin{tabular}{|c|c|c|}
\hline E08000035 & Leeds & UKE42 \\
\hline E08000036 & Wakefield & UKE45 \\
\hline E08000037 & Gateshead & UKC22 \\
\hline E09000001 & City of London & UKI31 \\
\hline E09000002 & Barking and Dagenham & UKI52 \\
\hline E09000003 & Barnet & UKI71 \\
\hline E09000004 & Bexley & UKI51 \\
\hline E09000005 & Brent & UKI72 \\
\hline E09000006 & Bromley & UKI61 \\
\hline E09000007 & Camden & UKI31 \\
\hline E09000008 & Croydon & UKI62 \\
\hline E09000009 & Ealing & UKI73 \\
\hline E09000010 & Enfield & UKI54 \\
\hline E09000011 & Greenwich & UKI51 \\
\hline E09000012 & Hackney & UKI41 \\
\hline E09000013 & Hammersmith and Fulham & UKI33 \\
\hline E09000014 & Haringey & UKI43 \\
\hline E09000015 & Harrow & UKI74 \\
\hline E09000016 & Havering & UKI52 \\
\hline E09000017 & Hillingdon & UKI74 \\
\hline E09000018 & Hounslow & UKI75 \\
\hline E09000019 & Islington & UKI43 \\
\hline E09000020 & Kensington and Chelsea & UKI33 \\
\hline E09000021 & Kingston upon Thames & UKI63 \\
\hline E09000022 & Lambeth & UKI45 \\
\hline E09000023 & Lewisham & UKI44 \\
\hline E09000024 & Merton & UKI63 \\
\hline E09000025 & Newham & UKI41 \\
\hline E09000026 & Redbridge & UKI53 \\
\hline E09000027 & Richmond upon Thames & UKI75 \\
\hline E09000028 & Southwark & UKI44 \\
\hline E09000029 & Sutton & UKI63 \\
\hline E09000030 & Tower Hamlets & UKI42 \\
\hline E09000031 & Waltham Forest & UKI53 \\
\hline E09000032 & Wandsworth & UKI34 \\
\hline E09000033 & Westminster & UKI32 \\
\hline E10000002 & Buckinghamshire & UKJ13 \\
\hline E10000003 & Cambridgeshire & UKH12 \\
\hline E10000006 & Cumbria & UKD11, UKD12 \\
\hline E10000007 & Derbyshire & UKF13, UKF12 \\
\hline E10000008 & Devon & UKK43 \\
\hline E10000011 & East Sussex & UKJ22 \\
\hline E10000012 & Essex & UKH37, UKH34, UKH35, UKH36 \\
\hline E10000013 & Gloucestershire & UKK13, UKK12 \\
\hline E10000014 & Hampshire & UKJ36, UKJ37, UKJ35 \\
\hline E10000015 & Hertfordshire & UKH23 \\
\hline E10000016 & Kent & UKJ43, UKJ44, UKJ45, UKJ46 \\
\hline E10000017 & Lancashire & UKD45, UKD46, UKD47, UKD44 \\
\hline E10000018 & Leicestershire & UKF22 \\
\hline E10000019 & Lincolnshire & UKE13, UKF30 \\
\hline
\end{tabular}




\begin{tabular}{|l|l|l|}
\hline E10000020 & Norfolk & UKH15, UKH17, UKH16 \\
\hline E10000021 & Northamptonshire & UKF24, UKF25 \\
\hline E10000023 & North Yorkshire & UKE22 \\
\hline E10000024 & Nottinghamshire & UKF15, UKF16 \\
\hline E10000025 & Oxfordshire & UKJ14 \\
\hline E10000027 & Somerset & UKK12, UKK23 \\
\hline E10000028 & Staffordshire & UKG24 \\
\hline E10000029 & Suffolk & UKH14 \\
\hline E10000030 & Surrey & UKJ25, UKJ26 \\
\hline E10000031 & Warwickshire & UKG13 \\
\hline E10000032 & West Sussex & UKJ28, UKJ27 \\
\hline E10000034 & Worcestershire & UKG12 \\
\hline
\end{tabular}

Table 11: Mapping between the Upper Tier Local Authority (UTLA) regions and the Nomenclature of Territorial Units for Statistics at level 3 codes (NUTS-3).

\section{B Baseline parameters for social contact rates}

This appendix outlines the sources used for the baseline social contact rate parameters. In particular, two sources have been used: the POLYMOD study [39], processed using the methodology of PyRoss [55], and the BBC Pandemic study [28] is used as a robustness check. It should be noted that these parameters are used as a baseline, and a further detailed calibration is carried out region by region to account for heterogeneity of social contact patterns across UK regions. We use estimates for social contact rates across the 16 age groups (detailed in Table 1) given in Mossong et al. [39]:

$$
\sigma=\left[\begin{array}{ccccccccccccccccc}
1.92 & 0.81 & 0.47 & 0.30 & 0.49 & 0.79 & 0.89 & 1.07 & 0.44 & 0.27 & 0.35 & 0.27 & 0.22 & 0.15 & 0.10 & 0.02 \\
0.78 & 6.64 & 1.24 & 0.58 & 0.49 & 0.72 & 1.09 & 1.40 & 1.10 & 0.36 & 0.35 & 0.23 & 0.35 & 0.24 & 0.07 & 0.23 \\
0.42 & 1.16 & 6.85 & 1.30 & 0.25 & 0.37 & 0.57 & 1.10 & 1.18 & 0.64 & 0.35 & 0.35 & 0.2 & 0.2 & 0.17 & 0.14 \\
0.26 & 0.52 & 1.26 & 6.71 & 1.24 & 0.72 & 0.47 & 0.87 & 0.97 & 0.97 & 0.52 & 0.31 & 0.2 & 0.26 & 0.24 & 0.28 \\
0.43 & 0.44 & 0.24 & 1.26 & 2.59 & 1.36 & 0.84 & 0.76 & 0.83 & 0.93 & 0.63 & 0.5 & 0.31 & 0.22 & 0.16 & 0.17 \\
0.73 & 0.68 & 0.38 & 0.76 & 1.42 & 1.83 & 1.13 & 0.92 & 0.9 & 0.92 & 0.85 & 0.72 & 0.45 & 0.38 & 0.18 & 0.12 \\
0.73 & 0.93 & 0.53 & 0.44 & 0.79 & 1.02 & 1.67 & 1.27 & 0.98 & 0.72 & 0.7 & 0.63 & 0.48 & 0.27 & 0.09 & 0.27 \\
0.79 & 1.06 & 0.89 & 0.74 & 0.63 & 0.74 & 1.12 & 1.5 & 1.27 & 0.86 & 0.63 & 0.55 & 0.53 & 0.43 & 0.14 & 0.31 \\
0.32 & 0.83 & 0.97 & 0.83 & 0.69 & 0.73 & 0.87 & 1.28 & 1.35 & 1.21 & 0.7 & 0.55 & 0.55 & 0.35 & 0.33 & 0.43 \\
0.24 & 0.32 & 0.62 & 0.96 & 0.91 & 0.87 & 0.75 & 1.02 & 1.41 & 1.87 & 0.75 & 0.64 & 0.51 & 0.26 & 0.32 & 0.33 \\
0.34 & 0.34 & 0.37 & 0.57 & 0.68 & 0.88 & 0.81 & 0.82 & 0.90 & 0.82 & 0.74 & 0.98 & 0.46 & 0.31 & 0.28 & 0.76 \\
0.24 & 0.20 & 0.35 & 0.32 & 0.50 & 0.69 & 0.67 & 0.66 & 0.66 & 0.66 & 0.91 & 1.17 & 0.73 & 0.43 & 0.20 & 0.46 \\
0.24 & 0.40 & 0.25 & 0.25 & 0.38 & 0.54 & 0.65 & 0.80 & 0.82 & 0.65 & 0.53 & 0.91 & 0.65 & 0.55 & 0.30 & 0.66 \\
0.19 & 0.31 & 0.29 & 0.39 & 0.32 & 0.52 & 0.42 & 0.74 & 0.60 & 0.39 & 0.42 & 0.63 & 0.64 & 0.70 & 0.52 & 0.20 \\
0.15 & 0.11 & 0.28 & 0.40 & 0.26 & 0.29 & 0.17 & 0.28 & 0.66 & 0.55 & 0.44 & 0.34 & 0.40 & 0.60 & 0.59 & 0.57 \\
0.01 & 0.18 & 0.12 & 0.24 & 0.14 & 0.10 & 0.24 & 0.32 & 0.43 & 0.28 & 0.60 & 0.39 & 0.45 & 0.12 & 0.29 & 0.86
\end{array}\right]
$$

We use the Bayesian hierarchical framework provided by the PyRoss library [2] to decompose contact rates into 'work', 'home', 'school', and 'other' 45]. The results are provided below, and also visualized in Figure 30. 


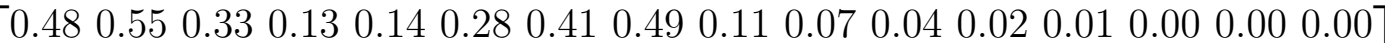
$\begin{array}{lllllllllllllllll}0.26 & 0.92 & 0.52 & 0.12 & 0.03 & 0.17 & 0.45 & 0.58 & 0.32 & 0.07 & 0.03 & 0.01 & 0.00 & 0.00 & 0.00 & 0.00\end{array}$ $\begin{array}{llllllllllllllllllll}0.17 & 0.54 & 1.08 & 0.39 & 0.04 & 0.01 & 0.22 & 0.59 & 0.49 & 0.13 & 0.04 & 0.02 & 0.00 & 0.01 & 0.00 & 0.00\end{array}$ $\begin{array}{lllllllllllllllllllll}0.09 & 0.15 & 0.42 & 0.98 & 0.13 & 0.03 & 0.06 & 0.25 & 0.42 & 0.21 & 0.07 & 0.05 & 0.01 & 0.02 & 0.00 & 0.00\end{array}$ $\begin{array}{llllllllllllllllll}0.17 & 0.08 & 0.07 & 0.36 & 0.80 & 0.21 & 0.07 & 0.04 & 0.16 & 0.28 & 0.08 & 0.09 & 0.02 & 0.01 & 0.00 & 0.00\end{array}$ $\begin{array}{lllllllllllllllll}0.49 & 0.30 & 0.04 & 0.07 & 0.13 & 0.66 & 0.21 & 0.03 & 0.01 & 0.05 & 0.17 & 0.11 & 0.03 & 0.00 & 0.00 & 0.00\end{array}$ $\begin{array}{lllllllllllllllllll}0.32 & 0.47 & 0.27 & 0.08 & 0.05 & 0.09 & 0.64 & 0.15 & 0.03 & 0.00 & 0.01 & 0.04 & 0.02 & 0.00 & 0.00 & 0.00\end{array}$ $\begin{array}{llllllllllllllll}0.38 & 0.70 & 0.56 & 0.20 & 0.02 & 0.01 & 0.09 & 0.59 & 0.15 & 0.00 & 0.02 & 0.01 & 0.01 & 0.01 & 0.00 & 0.00\end{array}$ $\begin{array}{llllllllllllllllll}0.17 & 0.52 & 0.73 & 0.42 & 0.07 & 0.00 & 0.09 & 0.19 & 0.46 & 0.10 & 0.02 & 0.00 & 0.03 & 0.01 & 0.01 & 0.00\end{array}$ $\begin{array}{llllllllllllllll}0.13 & 0.15 & 0.33 & 0.71 & 0.35 & 0.08 & 0.01 & 0.08 & 0.07 & 0.53 & 0.11 & 0.03 & 0.01 & 0.00 & 0.00 & 0.01\end{array}$ $\begin{array}{llllllllllllllll}0.12 & 0.10 & 0.18 & 0.26 & 0.31 & 0.07 & 0.07 & 0.04 & 0.08 & 0.06 & 0.37 & 0.13 & 0.01 & 0.00 & 0.00 & 0.00\end{array}$ $\begin{array}{lllllllllllllllll}0.02 & 0.01 & 0.11 & 0.28 & 0.23 & 0.18 & 0.06 & 0.02 & 0.01 & 0.08 & 0.09 & 0.39 & 0.10 & 0.00 & 0.00 & 0.00\end{array}$ $\begin{array}{llllllllllllllll}0.02 & 0.00 & 0.05 & 0.03 & 0.09 & 0.10 & 0.11 & 0.07 & 0.08 & 0.02 & 0.04 & 0.11 & 0.48 & 0.06 & 0.01 & 0.00\end{array}$ $\begin{array}{lllllllllllllllll}0.05 & 0.07 & 0.11 & 0.15 & 0.02 & 0.01 & 0.02 & 0.10 & 0.22 & 0.03 & 0.02 & 0.05 & 0.08 & 0.51 & 0.05 & 0.00\end{array}$ $\begin{array}{llllllllllllllll}0.00 & 0.02 & 0.16 & 0.25 & 0.01 & 0.00 & 0.00 & 0.00 & 0.30 & 0.08 & 0.03 & 0.00 & 0.06 & 0.14 & 0.16 & 0.09\end{array}$

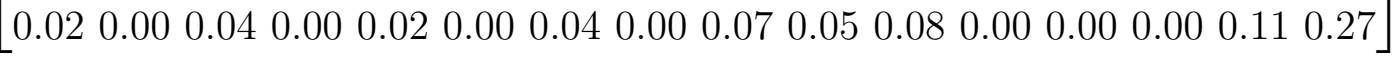

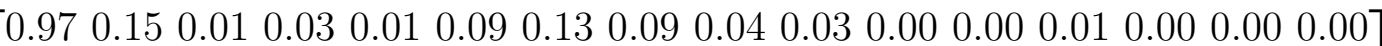
$\begin{array}{llllllllllllllll}0.24 & 2.35 & 0.06 & 0.00 & 0.02 & 0.04 & 0.06 & 0.06 & 0.06 & 0.05 & 0.04 & 0.00 & 0.00 & 0.00 & 0.00 & 0.00\end{array}$ $\begin{array}{lllllllllllllllllllll}0.00 & 1.12 & 2.56 & 0.12 & 0.01 & 0.05 & 0.04 & 0.12 & 0.09 & 0.07 & 0.03 & 0.02 & 0.01 & 0.00 & 0.00 & 0.00\end{array}$ $\begin{array}{llllllllllllllllllllll}0.04 & 0.08 & 1.17 & 4.14 & 0.06 & 0.12 & 0.08 & 0.08 & 0.07 & 0.05 & 0.04 & 0.03 & 0.00 & 0.00 & 0.00 & 0.00\end{array}$ $\begin{array}{lllllllllllllllll}0.00 & 0.13 & 0.00 & 0.27 & 0.23 & 0.01 & 0.02 & 0.03 & 0.01 & 0.01 & 0.01 & 0.00 & 0.00 & 0.00 & 0.00 & 0.00\end{array}$ $\begin{array}{lllllllllllllllllll}0.11 & 0.07 & 0.01 & 0.00 & 0.00 & 0.06 & 0.04 & 0.06 & 0.04 & 0.03 & 0.00 & 0.00 & 0.00 & 0.00 & 0.00 & 0.00\end{array}$ $\begin{array}{llllllllllllllll}0.00 & 0.10 & 0.01 & 0.01 & 0.01 & 0.00 & 0.04 & 0.00 & 0.02 & 0.00 & 0.00 & 0.00 & 0.00 & 0.00 & 0.00 & 0.00\end{array}$ $\begin{array}{lllllllllllllllllllllll}0.04 & 0.17 & 0.07 & 0.03 & 0.00 & 0.02 & 0.05 & 0.07 & 0.07 & 0.05 & 0.00 & 0.01 & 0.00 & 0.01 & 0.00 & 0.00\end{array}$ $\begin{array}{lllllllllllllllllll}0.07 & 0.10 & 0.03 & 0.00 & 0.04 & 0.06 & 0.03 & 0.06 & 0.02 & 0.01 & 0.01 & 0.01 & 0.00 & 0.00 & 0.00 & 0.00\end{array}$ $\begin{array}{llllllllllllllllllll}0.02 & 0.00 & 0.02 & 0.21 & 0.00 & 0.00 & 0.05 & 0.01 & 0.07 & 0.05 & 0.06 & 0.04 & 0.01 & 0.00 & 0.00 & 0.00\end{array}$ $\begin{array}{llllllllllllllll}0.00 & 0.00 & 0.00 & 0.00 & 0.00 & 0.00 & 0.00 & 0.00 & 0.02 & 0.00 & 0.00 & 0.04 & 0.00 & 0.00 & 0.00 & 0.00\end{array}$ $\begin{array}{llllllllllllllll}0.05 & 0.14 & 0.05 & 0.00 & 0.00 & 0.00 & 0.03 & 0.00 & 0.15 & 0.03 & 0.00 & 0.08 & 0.00 & 0.00 & 0.00 & 0.00\end{array}$ $\begin{array}{llllllllllllllllllll}0.00 & 0.13 & 0.00 & 0.00 & 0.02 & 0.01 & 0.05 & 0.11 & 0.02 & 0.03 & 0.01 & 0.01 & 0.00 & 0.00 & 0.00 & 0.00\end{array}$ $\begin{array}{llllllllllllllllll}0.00 & 0.06 & 0.04 & 0.00 & 0.00 & 0.00 & 0.04 & 0.03 & 0.00 & 0.00 & 0.00 & 0.03 & 0.00 & 0.00 & 0.00 & 0.00\end{array}$

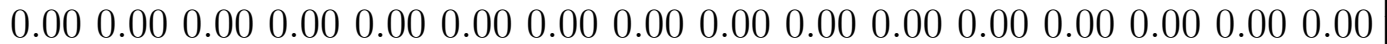

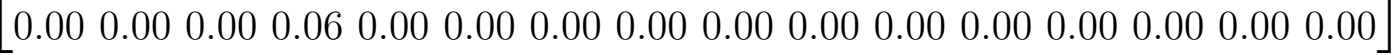

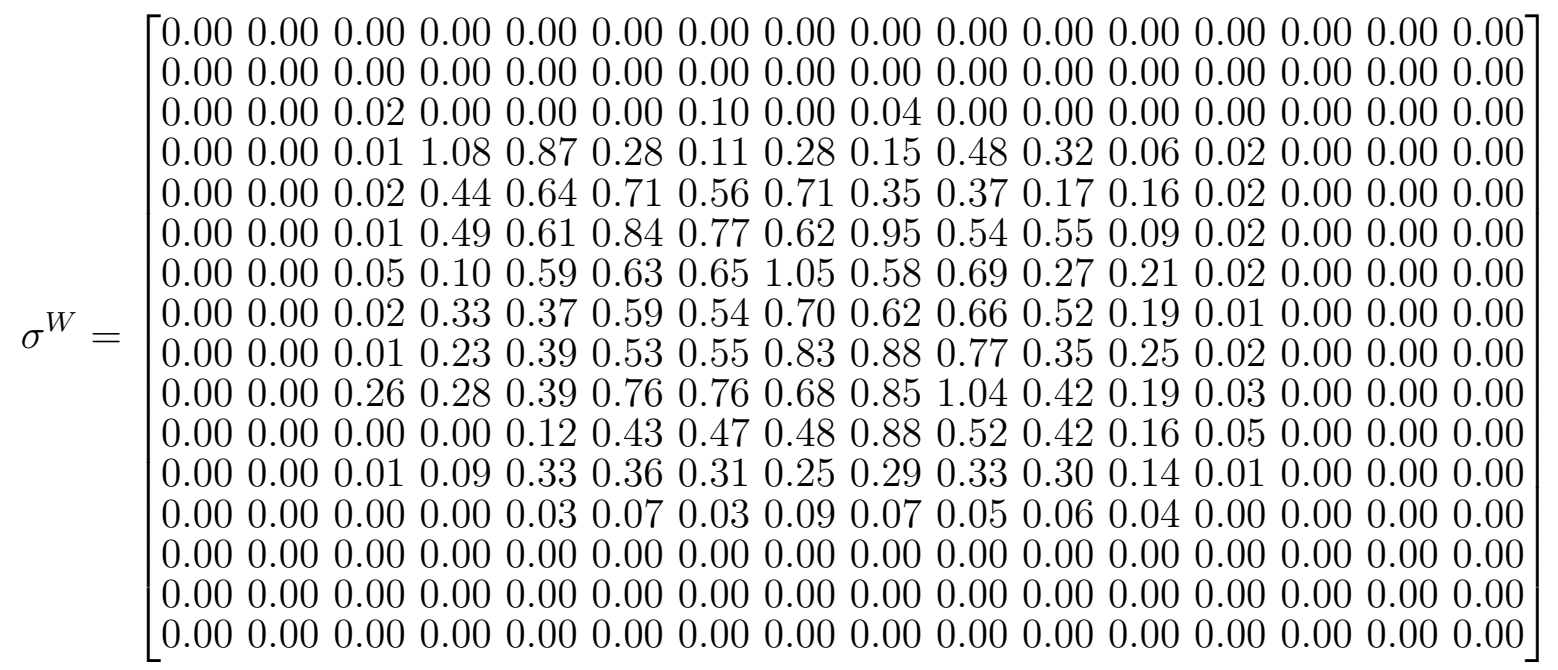




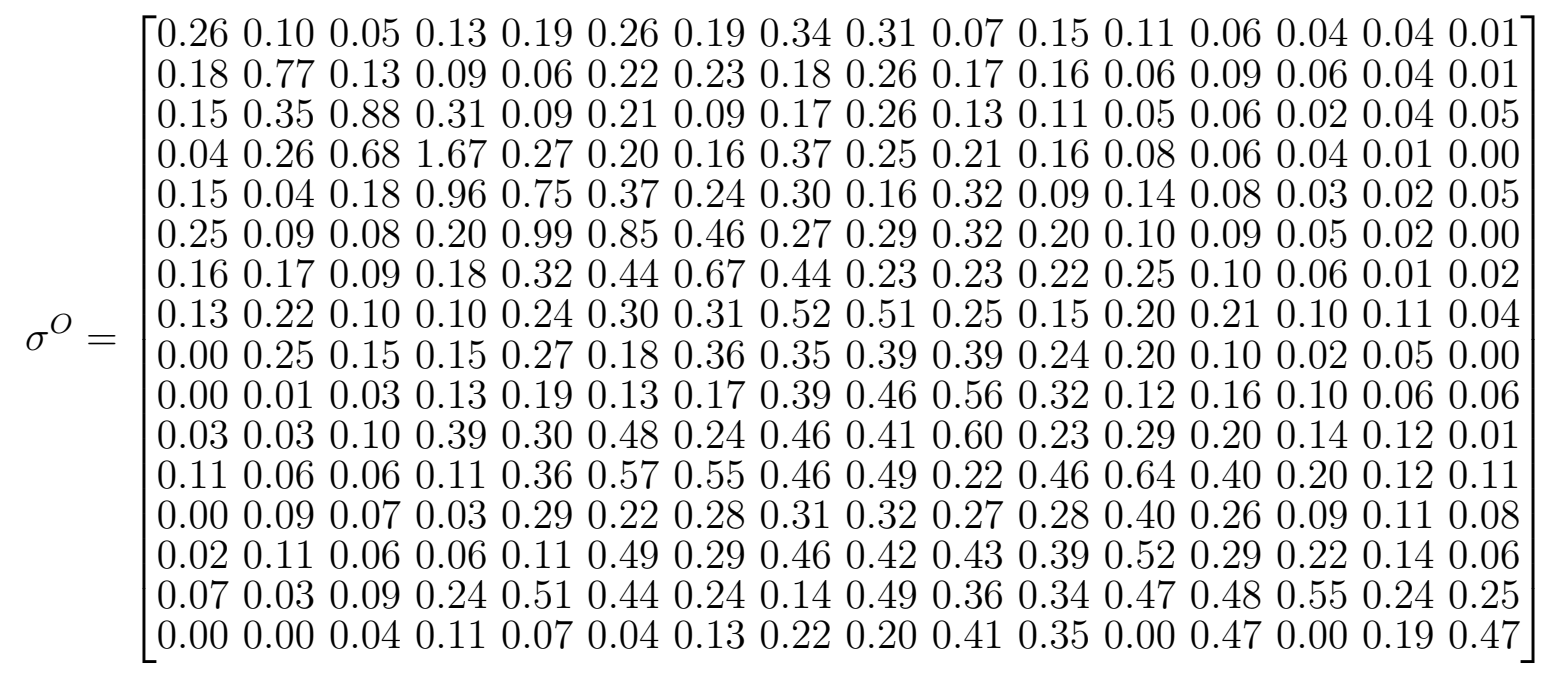

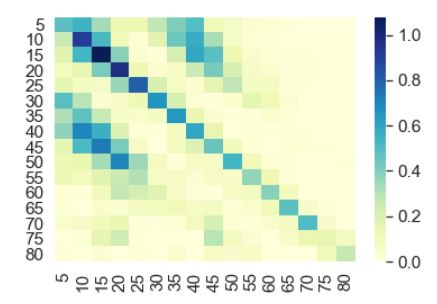

(a) Home.

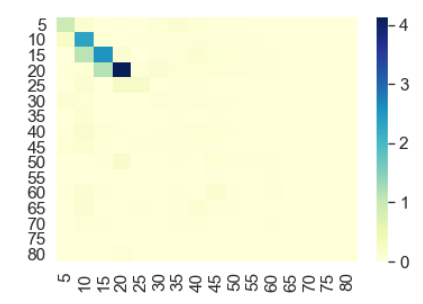

(b) School.

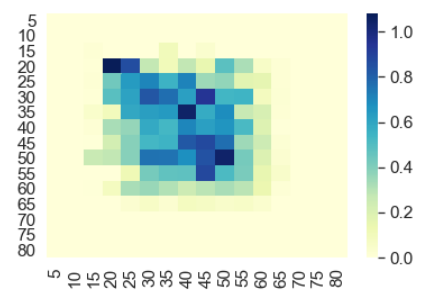

(c) Work.

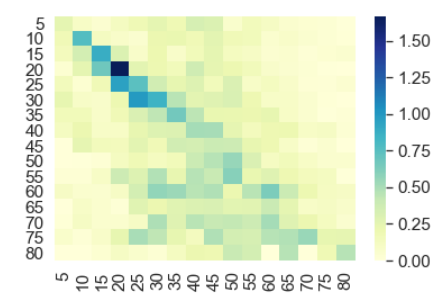

(d) Other.

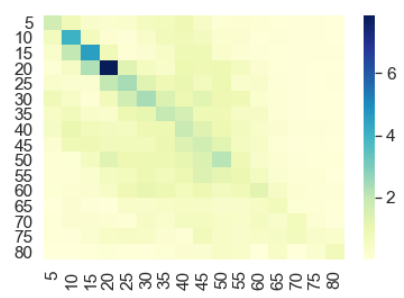

(e) Total.

Figure 30: Baseline social contact matrices with 16 age groups.

Note that these baseline values are modulated to reflect regional differences, using the approach described in Section 3 . 